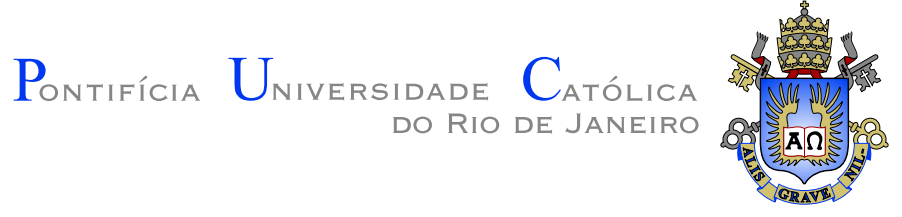

Dimas Leão Ramos

\title{
Robust Portfolio Optimization Under Conflicting Views: a Black-Litterman Model Approach
}

Dissertation presented to the Programa de Pós-Graduação em Engenharia de Produção of the Departamento de Engenharia Industrial, PUC-Rio as partial fulfillment of the requirements for the degree of Mestre em Engenharia de Produção.

Advisor

Prof. Davi Michel Valladão

Co-advisor: Prof. Betina Dodsworth Martins Froment Fernandes 


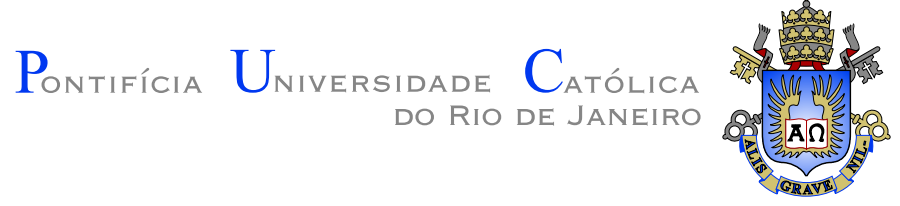

Dimas Leão Ramos

\section{Robust Portfolio Optimization Under Conflicting Views: a Black-Litterman Model Approach}

Dissertation presented to the Programa de Pós-Graduação em Engenharia de Produção in partial fulfillment of the requirements for the degree of Mestre em Engenharia de Produção. Approved by the undersigned Examination Committee.

Prof. Davi Michel Valladão

Advisor

Departamento de Engenharia Industrial - PUC-Rio

Prof. Betina Dodsworth Martins Froment Fernandes Co-advisor MBA em Gestão de Investimentos - IAG/PUC-Rio

Prof. Thuener Armando da Silva Departamento de Engenharia Industrial - PUC-Rio

Prof. Rafael Martinelli Pinto Departamento de Engenharia Industrial — PUC-Rio

Prof. Márcio da Silveira Carvalho Coordinator of the Centro Técnico Científico - PUC-Rio 
All rights reserved.

\section{Dimas Leão Ramos}

Dimas Leão Ramos currently is a master student candidate of Industrial Engineering departament at PUC-Rio. His research focus on Robust Optimization for porfolio allocation problems. He undergraduated in Civil Engineering at Federal University of Alagoas in 2014.

Bibliographic data

Leão Ramos, Dimas

Robust Portfolio Optimization Under Conflicting Views: a Black-Litterman Model Approach / Dimas Leão Ramos; advisor: Davi Michel Valladão; co-advisor: Betina Dodsworth Martins Froment Fernandes. - 2017.

103 f. : il. (color.); $30 \mathrm{~cm}$

Dissertação (mestrado) - Pontifícia Universidade Católica do Rio de Janeiro, Rio de Janeiro, Departamento de Engenharia Industrial, 2017.

Inclui bibliografia.

1. Engenharia Industrial - Teses. 2. Otimização Robusta. 3. Modelo Black-Litterman. 4. Otimização de Portfólio. 5. Finanças. I. Michel Valladão, Davi. II. Dodsworth Martins Froment Fernandes, Betina. III. Pontifícia Universidade Católica do Rio de Janeiro. Departamento de Engenharia Industrial. IV. Título. 


\section{Acknowledgement}

Thank to my advisors Prof. Davi Valladão and Profa. Betina Fernandes for their support and encouragement for this work.

To my parents Viviane and Adeildo, for their unconditional support during all this journey.

To my family and friends who supported me even with my absence in family life.

To my girlfirend Fernanda, who accompanied me all this time with direct and indirect support.

To all colleagues of the Laboratoy of Applied Mathematical Programming and Statistics (LAMPS), for the great work atmosphere and fun moments they created in the laboratory.

To all colleagues, faculty and staff of the Department of PUC-Rio, for the fellowship, learning and support.

Thank to $\mathrm{CNPq}$ for the financial support which allowed this work to be done. 


\section{Abstract}

Leão Ramos, Dimas; Michel Valladão, Davi (Advisor); Dodsworth Martins Froment Fernandes, Betina (Co-Advisor). Robust Portfolio Optimization Under Conflicting Views: a BlackLitterman Model Approach. Rio de Janeiro, 2017. 103p. Dissertação de Mestrado — Departamento de Engenharia Industrial, Pontifícia Universidade Católica do Rio de Janeiro.

Black and Litterman proposed a portfolio optimization model that combines investor's views on future asset's returns with neutral market equilibrium. However, specifying portfolio views is a challenging task, specially when investors have conflicting opinions on the same asset. In this thesis, we suggest a new portfolio optimization formulation that is robust for investor's views. Our approach was tested on synthetic and real data available on a framework developed by Central Bank of Brazil. This online framework collects projections on main macroeconomics variables from more than a hundred professional forecasters and provides public online access on a weekly basis. The performance of this new robust formulation is compared with the traditional Black-Litterman model. The result show that our robust methodology can provide better risk adjusted performance compared to the orignial model and are less sensitive to incorrect inverstor views.

\section{Keywords}

Robust Optimization; Portfolio Optimization; Black-Litterman Model; Finance. 


\section{Resumo}

Leão Ramos, Dimas; Michel Valladão, Davi; Dodsworth Martins Froment Fernandes, Betina. Otimização de Portfólio Robusta Sob Visões Conflitantes: Uma Abordagem BlackLitterman. Rio de Janeiro, 2017. 103p. Dissertação de Mestrado - Departamento de Engenharia Industrial, Pontifícia Universidade Católica do Rio de Janeiro.

Black e Litterman propuseram um modelo de otimização de portfólio que combina visões do investidor sobre retornos esperados de ativos com o equilíbrio neutro de mercado. No entanto, especificar visões sobre uma carteira de investimentos é uma tarefa difícil, especialmente quando os investidores têm opiniões conflitantes sobre o mesmo ativo. Neste trabalho, é proposto uma nova formulação para otimização de carteiras, que é robusta diferentes à visões do investidor. A nossa abordagem foi testada em dados sintéticos e dados reais disponíveis em uma plataforma do Banco Central do Brasil. Esta plataforma consolida projeções macroeconômicas de mais de uma centena de analistas profissionais e disponibiliza para o mercado numa base semanal. Por fim, é comparado o desempenho desta formulação robusta com o modelo Black-Litterman tradicional frequentemente utilizado na indústria financeira. Os resultados mostram que a metodologia robusta pode providenciar melhor desempenho ajustado ao risco em comparação com o modelo orignial e são menos sensíveis às visões do investor.

\section{Palavras-chave}

Otimização Robusta; Modelo Black-Litterman; Otimização de Portfólio; Finanças. 


\section{Contents}

1 Introduction $\quad 10$

$\begin{array}{lll}1.1 & \text { Contributions } & 11\end{array}$

$\begin{array}{lll}1.2 & \text { Organization } & 12\end{array}$

$\begin{array}{lll}1.3 & \text { Notation } & 13\end{array}$

2 Literature Review 14

2.1 Second-order Cone Programming 14

2.2 Robust Optimization 21

2.3 Black-Litterman Model 31

3 Proposed Robust Model based on Black-Litterman Approach $\quad 40$

3.1 General Robust Black-Litterman Model 40

3.2 Black-Litterman with Multiple Forecasters 41

3.3 Robust Black-Litterman with Incomplete Information 44

4 Controlled Tests $\quad 51$

4.1 Experiment Setup $\quad 51$

4.2 Sensitivity to Uncertainty of the Views 56

4.3 Sensitivity to Accuracy of the Forecasters 62

5 Market Expectations System Backtest $\quad 68$

5.1 Market Expectations System 68

5.2 Interest Rate Forecast to Returns 69

5.3 Data and Assumptions 73

$\begin{array}{lll}5.4 & \text { Results } & 77\end{array}$

$\begin{array}{llr}6 & \text { Conclusions } & 89\end{array}$

7 Bibliography $\quad 90$

A Tables from Synthetic Data Experiments $\quad 96$ 


\section{List of figures}

2.1 Illustration of a $L_{3},\left\{\left(u_{1}, u_{2}, t\right) \mid\left(u_{1}+u_{2}\right)^{1 / 2} \leq t\right\} \quad 16$

2.2 Example of an ellipsoidal uncertainty set 26

2.3 Example of Bertsimas uncertainty. On the left: $\Gamma=2$, on the right: $\Gamma=1$.

2.4 Example of CVaR uncertainty set. 28

2.5 Example of robust loss constraint in different market conditions. 31

2.6 Black-Litterman allocation model with and without views 38

3.1 Classical and Robust Black-Litterman allocations 44

3.2 Example of Black-Litterman model using confidence ellipsoid. On the left: $\mathrm{N}=2$, on the right: $\mathrm{N}=10$.

3.3 Efficient frontier for $\alpha=0.95$.

4.1 Confidence interval for $\tau_{\mathbf{q}}$. 54

$\begin{array}{lll}4.2 & \text { Average return } \times \tau_{\mathbf{q}} & 57\end{array}$

4.3 Empirical probability $\times \tau_{\mathbf{q}} \quad 58$

4.4 Out of sample standard deviation and portfolio returns without bias views for $\tau_{\mathbf{q}}=5$.

4.5 Out of sample standard deviation and portfolio returns with bias views for $\tau_{\mathbf{q}}=5$.

4.6 Sharpe Ratio $\times \tau_{\mathbf{q}}$

4.7
4.8 Average return of the simulations $\times \xi_{\mathbf{q}}$

4.9 Standard deviation of the simulations $\times \xi_{\mathbf{q}}$

4.10 Sharpe ratio $\times \xi_{\mathbf{q}} \quad 65$

4.11 Empirical probability of the simulations $\times \xi_{\mathbf{q}} \quad 66$

5.1 Implied yield curves from the forecasts and the nominal yield curve at $02 / 07 / 2016$.

5.2 Cumulative returns of the asset classes from March 2010 until October 2016

5.3 Market portfolio from September 2003 to October 2016.

5.4 Boxplots of the out of sample daily returns for all allocation methodologies using $\tau_{c}=1$ and $\tau_{c}=100$.

5.5 All strategies cumulative return for $\tau_{c}=(0.1,1,10,100)$. $\quad 80$

5.6 Portfolio weights in the period of analysis for $\tau_{c}=0.1$. 81

5.7 Portfolio weights in the period of analysis for $\tau_{c}=1 . \quad 82$

5.8 Portfolio weights in the period of analysis for $\tau_{c}=10$. 83

5.9 Portfolio weights in the period of analysis for $\tau_{c}=100$. 84

5.10 Boxplot of the monthly portfolio weights for $\tau_{c}=1$. 86

5.11 Boxplot of the monthly portfolio weights for $\tau_{c}=100$. 87 


\section{List of tables}

5.1 Comparison of implied SELIC rates and its forecasts from the Market Expectations system.

5.2 Expected return using the Market Expectations system as views

5.3 Annualized average return and standard deviation from all strategies. All results are shown in percentage.

5.4 Average portfolio weights on the data set for $\tau_{c}=(0.1,1,10,100)$.

A.1 This table reports the mean, standard deviation, maximum, minimum, empirical probability and ratio of the controlled experiments for the original Black-Litterman using the average of all 5 forecasters 97

A.2 This table reports the mean, standard deviation, maximum, minimum, empirical probability and ratio of the controlled experiments for the robust Black-Litterman with perfect information on all forecasters

A.3 This table reports the mean, standard deviation, maximum, minimum, empirical probability and ratio of the controlled experiments for the robust Black-Litterman for the ellipsoidal uncertainty set on the average return of the posterior distribution.

A.4 This table reports the mean, standard deviation, maximum, minimum, empirical probability and ratio of the controlled experiments for the robust Black-Litterman for the ellipsoidal uncertainty set of the average views.

A.5 This table reports the mean, standard deviation, maximum, minimum, empirical probability and ratio of the controlled experiments for the robust Black-Litterman using Bertsimas and Sim's uncertainty set for $\Gamma=1$

A.6 This table reports the mean, standard deviation, maximum, minimum, empirical probability and ratio of the controlled experiments for the robust Black-Litterman using Bertsimas and Sim's uncertainty set for $\Gamma=2$

A.7 This table reports the mean, standard deviation, maximum, minimum, empirical probability and ratio of the controlled experiments for the robust Black-Litterman for the ellipsoidal uncertainty set of the views with information on its covariance matrix. 


\section{Introduction}

In 1952 Professor Harry Markowitz published one of his most notorious work named "Portfolio Selection" (Markowitz, 1952), which is considered one of the main articles in quantitative finance and states the beginning of modern portfolio theory. His innovative approach goes beyond the traditional asset management, which focused on predicting stock price changes using fundamental and technical analysis. According to Markowitz, portfolio selection problem consists on finding the optimal trade off between risk and return. Moreover, his results form the theoretical foundation of a concept that practitioners and academics have always known, that diversification reduces risk on a given portfolio.

Markowitz's model requires distribution information concerning the behavior of future assets returns. However, returns are not completely known by academics or practitioners, therefore approximate return models are used to describe its dynamics. Thus, in order to implement the mean-variance approach proposed by Markowitz, one needs to estimate means and covariances of asset returns and plug these estimates into an optimization problem modeled by the investor. This leads to an important drawback of the conventional mean-variance approach, the estimation error from data samples. However, a significant number of researchers have tried to diminish the impact of estimation errors in the optimal allocation (see DeMiguel et al. (2009); Chopra and Ziemba (1993); Best and Grauer (1992)). Some of the techniques proposed are portfolio re-sampling and Bayesian shrinkage, for more details on the topic we refer to Jorion (1986) and Basak et al. (2009).

These practical drawbacks motivated Fisher Black and Robert Litterman while working at Goldman Sachs to develop a new asset allocation methodology. As a result, the idea to combine equilibrium estimates of asset returns with investor's private opinions about future returns was introduced at Black and Litterman (1992). Their approach employed a Bayesian analytical methodology to estimate new asset returns and a covariance matrix. Computational tests have shown that the optimal portfolios resulted by this method are more intuitive, stable and diversified, when compared to the conventional Markowitz methodology. As a result, the model has found much favor with practitioners. In the Brazilian financial market, robot advisor firms (Vérios, Warren) have published on their website that they use the Black-Litterman model at the 
core of their investment analytics. For further details on the model see Walters (2011).

Black and Litterman's original paper (Black and Litterman, 1992) only explained the main ideas, leaving it to other researchers to better explain the implications of their model. Subsequent research on the Black-Litterman model was done by Satchell and Scowcroft (2000); Walters (2011); He and Litterman (2002), where they provide a more complete survey on the model and explains it in further detail. Also, a complete applied perspective of the Black-Litterman model was conducted by Mankert (2010). Other authors have focused on extensions of the original model, as in Herold (2005); Idzorek (2002); Fernandes et al. (2013); Meucci (2008); Silva et al. (2017).

A very dynamic area of research in asset management is robust portfolio optimization. This approach acknowledges the impacts of estimation error and seeks for the optimal portfolio under the worst-case realizations of estimation uncertainty. Among many studies on portfolio robust optimization, Lobo and Boyd (2000) provide an introduction to robust portfolio optimization formulations, listing uncertainty sets that are convex and tractable to model asset returns. Moreover, Halldórsson and Tütüncü (2003) introduce a robust formulation for the mean-variance model, that allocates the solution in the worst-case performance within the set of values for the mean and covariance matrix in the uncertainty set. More recently, Fernandes et al. (2016), proposed a new adaptive robust portfolio model. Their asset allocation model uses datadriven polyhedral uncertainty sets to construct robust loss constraints on a rolling horizon scheme. Moreover, through empirical results using realistic transaction costs in the Brazilian Market, they show that this new strategy can introduce a new perspective of robust optimization for industry practitioners. For a thorough discussion related to robust portfolio management see Fabozzi et al. (2007), Kim et al. (2013), Fabozzi et al. (2009) and Fernandes et al. (2016).

\section{1}

\section{Contributions}

The objective of portfolio managers is to achieve results beyond market benchmarks by using information and techniques that is not broadly available to general investors. In this thesis we provide a robust optimization approach on the Black-Litterman model that can significantly improve the performance and risk management of practitioners. We summarize the main contributions of this thesis as follows:

1. Using concepts from robust optimization, we propose a general robust 
allocation model based on the Black-Litterman framework. In particular, our framework enables to incorporate robustness through uncertainty sets on the views from different forecasters and on the market model.

2. We provide computational evidence using synthetic data that robust black-litterman portfolios can present better risk-adjusted performance profiles compared to the original model. By introducing robustness on views, we empirically show that the overall performance of the portfolios are less sensitive to accuracy on portfolio views. We also show that incorporating the overall uncertainty structure of multiple forecasters can improve portfolio allocation.

3. Our framework was tested using real market data from the asset classes available to Brazilain investors. The views were modeled using a database of macroeconomics forecasts from market participants provided by the Central Bank of Brazil. The results suggests using the total uncertainty structure of market views from different experts can provide a better out of sample risk-reward performance compared to the original BlackLitterman model.

\section{2 \\ Organization}

The remainder of this thesis is structured as follows. In Chapter 2 we review the theoretical background. First, we introduce the general setting of second order cone optimization problems that represents the foundations of the models we further propose. Then, we discuss recent advances on robust portfolio optimization. We show that the modified original problem can be transformed into a continuous and convex problem using robust optimization techniques. Finally, we briefly discuss the Black-Litterman Model and set an asset allocation example using the model.

Chapter 3 derives the robust allocation models based on the BlackLitterman approach under the assumption of conflicting information on the views and shows a simple example of how to use these techniques in practice. Chapter 4 provides numerical results illustrating the capabilities of these new models on synthetic data. Chapter 5 we use real market data and source of conflicting views from the Market Expectation System to backtest the robust models we have proposed. Finally, Chapter 6 contains our conclusions on the work shown in this thesis. 


\section{3}

\section{Notation}

Througout this article we use bold characters to represent vectors and matrices. Matrices are denoted by upper case letters (e.g. A), lower case letters (e.g. a) are used to represent vectors and ordinary letters (e.g. $R, t, \epsilon, \ldots$ ) to represent scalars. Boldface are also used for column and row vectors of a matrix, for instance $\mathbf{A}_{i}$ stands for the $i$ th column vector of the matrix $\mathbf{A}$, and lower case $\mathbf{a}_{i}$ are used for the $i$ th row vector. Apostrophe (e.g. $\mathbf{a}^{\prime}$ ) is used for transposed matrix or vector. Hat (e.g. $\hat{a}$ ) denotes the nominal value of a. The bold character number 1 denotes a vector of ones. Overline (e.g. $\bar{a}$ ) and underline (e.g. $\underline{a}$ ) denote the upper and lower bound of a given variable. To represent identity matrix of dimension $n$ is used $\mathbf{I}_{n}$. Finally, o denotes element-wise products between matrices or vectors, also known as the Hadamard product. 


\section{2}

\section{Literature Review}

In this chapter, we present and discuss the theoretical background for the current work. The literature review is organized in three major sections. The first covers a review of second-order cone programming. The following section reviews robust optimization techniques and relates it to the discussion associated with second-order cone programming problems. Finally, we present the Black-Litterman method and recent relevant extensions of the method.

\section{1}

\section{Second-order Cone Programming}

Many problems in financial applications are designed as convex problems, in particular a wide variety of practical problems can be formulated as secondorder cone programming (SOCP). A few examples of SOCPs that occur in finance include variants of Markowitz asset allocation framework, problems with loss constraints and portfolio problems with transaction cost models. In this section our objective is to present an overview on second-order cone programming. Here we follow the work done in Lobo et al. (1998), Fabozzi et al. (2007), Lutgens (2004) and Nemirovski (2013) on second-order cone programming and conic programming.

Second-order cone programming is a class of conic optimization problems that generalizes linear and quadratic programming. Some of the problems that can be modeled as an SOCPs include quadratic constrained quadratic programming (QCQP), sum of norms and maximum of norms and hyperbolic constraints. Moreover, as linear programming (LP), SOCPs can be solved efficiently using primal-dual interior point methods.

First, consider a general conic programming (CP) problem

$$
\begin{array}{cl}
\underset{\mathbf{x}}{\operatorname{minimize}} & \mathbf{f}^{\prime} \mathbf{x} \\
\text { subject to } & \mathbf{A x}=\mathbf{b} \\
& \mathbf{x} \succeq_{C} 0,
\end{array}
$$

where $C$ is a proper cone (i.e. closed, convex, solid and pointed), $\mathbf{x} \in \mathbb{R}^{n}$ is the decision variable, and $\mathbf{f} \in \mathbb{R}^{n}, \mathbf{b} \in \mathbb{R}^{m}$ and $\mathbf{A} \in \mathbb{R}^{m \times n}$ are the parameters related to the problem. If $C$ is defined properly, this standard form can represent any convex optimization problem. For instance, when $C$ is taken 
as the nonnegative orthant $\left(\mathbb{R}_{+}^{n}\right)$ the problem reduces to a linear optimization problem.

The vector inequality $\mathbf{x} \succeq_{C} 0$ is often used to define a generalized inequality. For a proper cone $C$, this inequality induces a partial ordering on $\mathbb{R}^{n}$ which satisfies some of the properties on the standard ordering of the reals. From the definition of this ordering we have that

$$
\mathbf{x}-\mathbf{y} \succeq_{C} 0 \Longleftrightarrow \mathbf{x} \succeq_{C} \mathbf{y} \Longleftrightarrow \mathbf{x}-\mathbf{y} \in C
$$

Here we mention a few of the basic properties that is satisfied by a generalized inequality $\succeq_{C}$

- Transitivity: if $\mathbf{y} \succeq_{C} \mathbf{x}$ and $\mathbf{z} \succeq_{C} \mathbf{y}$ then $\mathbf{z} \succeq_{C} \mathbf{x}$;

- Reflexivity: $\mathbf{x} \succeq_{C} \mathbf{x}$;

- Anti-symmetry: if $\mathbf{y} \succeq_{C} \mathbf{x}$ and $\mathbf{x} \succeq_{C} \mathbf{y}$ then $\mathbf{x}=\mathbf{y}$;

- Homogeneity: if $\mathbf{x} \succeq_{C} \mathbf{y}$ and $\alpha$ is a nonnegative scalar then $\alpha \mathbf{x} \succeq_{C} \alpha \mathbf{y}$;

- Additivity: if $\mathbf{y} \succeq_{C} \mathbf{x}$ and $\mathbf{z} \succeq_{C} \mathbf{v}$ then $\mathbf{y}+\mathbf{z} \succeq_{C} \mathbf{v}+\mathbf{x}$.

Observe that when $C$ is defined as the nonnegative orthant (i.e. $C=$ $\mathbb{R}_{+}^{n}$ ), the associated generalized inequality $\mathbf{x} \succeq_{\mathbb{R}_{+}^{n}} \mathbf{y}$ is equivalent to an ordinary component-wise inequality between vectors, meaning that $x_{i} \geq y_{i}$. Furthermore, in $\mathrm{CP}$ a specific cone $C$ is associated to conic problem in the form of equation (2-1). Thus, the only difference between this program and an LP problem is the particular choice of cone and its implications. Also, the $\mathrm{CP}$ formulation allows to represent a broader class of problems that cannot be done by a LP problem. Here we restrict our applications to SOCP.

In a second-order cone programming, problem (2-1) is minimized over the cartesian products of second-order cones (which is also known as Lorentz cone, ice-cream cone and quadratic cone), where the standard second-order cone with dimension $\mathrm{k}$ has the form

$$
L_{k}=\left\{\left[\begin{array}{l}
\mathbf{u} \\
t
\end{array}\right] \mid \mathbf{u} \in \mathbb{R}^{k-1}, t \in \mathbb{R}_{+},\|\mathbf{u}\|_{2} \leq t\right\} .
$$

In figure 2.1 we illustrate a second-order cone in $\mathbb{R}^{3}$, note that by intersecting it with a hyperplane at different angles one obtain spherical and ellipsoidal sets. More interestingly, we will show that any quadratic constraint can be represented by a second-order cone. 


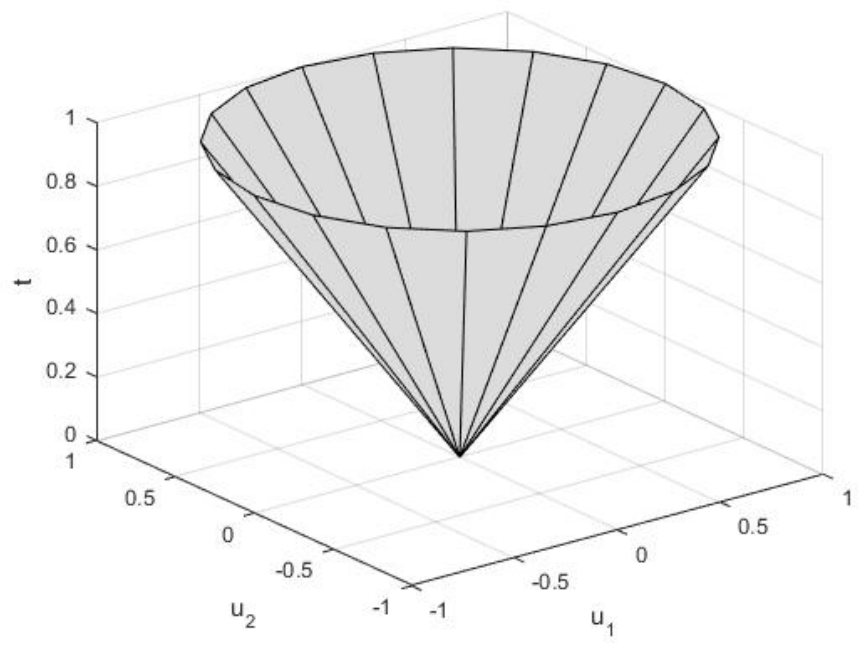

Figure 2.1: Illustration of a $L_{3},\left\{\left(u_{1}, u_{2}, t\right) \mid\left(u_{1}+u_{2}\right)^{1 / 2} \leq t\right\}$

Formally, the a second-order cone optimization problem is expressed when problem (2-1) takes the following form

$$
\begin{aligned}
\underset{\mathbf{x}}{\operatorname{minimize}} & \mathbf{f}^{\prime} \mathbf{x} \\
\text { subject to } & \left\|\mathbf{A}_{i} \mathbf{x}+\mathbf{b}_{i}\right\|_{2} \leq \mathbf{c}_{i}^{\prime} \mathbf{x}+d_{i}, i=1, \ldots, N,
\end{aligned}
$$

where $\mathbf{x} \in \mathbb{R}^{m}$ are the decision variables, and $\mathbf{b}_{i} \in \mathbb{R}^{n_{i}}, d_{i} \in \mathbb{R}, \mathbf{A}_{i} \in \mathbb{R}^{n_{i} \times m}$ and $\mathbf{c}_{i} \in R^{n_{i}}$ are the parameters associated to the problem. In addition, the constraint $\left\|\mathbf{A}_{i} \mathbf{x}-\mathbf{b}_{i}\right\|_{2} \leq \mathbf{c}_{i}^{\prime} \mathbf{x}-d_{i}$ is denoted as an second-order constraint, since satisfying this constraint is the same as restricting an affine function to lie in a unit second order cone of dimension $k+1$

$$
\left\|\mathbf{A}_{i} \mathbf{x}+\mathbf{b}_{i}\right\|_{2} \leq \mathbf{c}_{i}^{\prime} \mathbf{x}+d_{i} \Longleftrightarrow\left[\begin{array}{c}
\mathbf{A}_{i} \mathbf{x}+\mathbf{b}_{i} \\
\mathbf{c}_{i}^{\prime} \mathbf{x}+d_{i}
\end{array}\right] \in L_{k+1} .
$$

This class of conic optimization problem can be used to represent several usual convex programming problems. For instance, a special type of second order cone is the linear cone, which is defined for $k=1$ and the unit second order cone becomes $L_{1}=\left\{t \mid t \in \mathbb{R}_{+}, t \geq 0\right\}$. Thus, by taking $L_{n_{i}}=L_{1}$, for each constraint $i=1, \ldots, N$, the standard SOCP in problem (2-4) becomes a LP

$$
\begin{array}{ll}
\underset{\mathbf{x}}{\operatorname{minimize}} & \mathbf{f}^{\prime} \mathbf{x} \\
\text { subject to } & \mathbf{c}_{i}^{\prime} \mathbf{x}+d_{i} \geq 0, i=1, \ldots, N .
\end{array}
$$

Moreover, another class of problems that can be modeled as SOCPs is the quadratically constrained linear programming (QCLP). By assuming that the $i$ th $c_{i}=0$ for all constraints $i=1, \ldots, N$, it becomes equivalent convex 
quadratic constraints and the problem reduces to a quadratically constrained linear program

$$
\begin{array}{ll}
\underset{\mathbf{x}}{\operatorname{minimize}} & \mathbf{f}^{\prime} \mathbf{x} \\
\text { subject to } & \left\|\mathbf{A}_{i} \mathbf{x}+\mathbf{b}_{i}\right\|_{2}^{2} \leq d_{i}^{2}, i=1, \ldots, N,
\end{array}
$$

From these two problem transformations, it is clear that the SOCPs problems are more general than LPs and QCLPs. In fact, many other nonlinear convex optimization problems can formulated into SOCPs as well.

\subsection{1}

\section{SOCP Dual Problem}

Duality theory arise in mathematical programming as a methodology to find the best bound on the optimal value of an optimization problem. Often this original problem is known as primal and the one that its solution provides the best bound is called dual problem. The difference between their optimal values is called dual gap. Under special circumstances the duality gap is zero for convex optimization problems.

To illustrate, as a first example consider a standard LP as the primal problem

$$
\begin{array}{ll}
\underset{\mathbf{x}}{\operatorname{minimize}} & \mathbf{c}^{\prime} \mathbf{x} \\
\text { subject to } & \mathbf{A x} \geq \mathbf{b},
\end{array}
$$

here this would be considered the primal problem and its optimal is denoted as $p^{*}$. Furthermore, applying an inner product on both sides of the inequality in problem (2-8), we arrive at the following

$$
\langle\mathbf{y}, \mathbf{A x}\rangle \geq\langle\mathbf{y}, \mathbf{b}\rangle \Longleftrightarrow \mathbf{y}^{\prime} \mathbf{A} \mathbf{x} \geq \mathbf{y}^{\prime} \mathbf{b}
$$

where the elements of the vector $\mathbf{y}$ are nonnegative. This is a general result for LP problems and this inequality actually holds for every feasible solution of problem (2-8). Moreover, when the left hand side of the inequality (2-9) is equal to the objective function, i.e. $\mathbf{c}^{\prime} \mathbf{x}$, which is whenever the given equality is satisfied for all nonnegative $\mathbf{y}$

$$
\mathbf{A}^{\prime} \mathbf{y}=\mathbf{c}
$$

the result of inequality (2-9) lead to a lower bound on the objective function of (2-8) for all decision variables $\mathbf{x}$. As a result, it is also a lower bound to the optimal value $p^{*}$

$$
\mathbf{c}^{\prime} \mathbf{x} \geq p^{*} \geq \mathbf{y}^{\prime} \mathbf{b}
$$

The dual problem is defined as the optimization problem that yields the best lower bound of $p^{*}$. Moreover, we are interested in maximizing $\langle\mathbf{y}, \mathbf{b}\rangle$ 
satisfying the relation given on the scalar inequality (2-11), which is equivalent to solving the following problem

$$
\begin{array}{cl}
\underset{\mathbf{y}}{\operatorname{maximize}} & \mathbf{y}^{\prime} \mathbf{b} \\
\text { subject to } & \mathbf{A}^{\prime} \mathbf{y}=\mathbf{c} \\
& \mathbf{y} \geq 0 .
\end{array}
$$

An analog methodology can be applied to develop the dual problem of other conic programming problems, including SOCP. First, consider a rewritten form of problem (2-8) as a generic CP problem

$$
\begin{array}{ll}
\underset{\mathbf{x}}{\operatorname{minimize}} & \mathbf{c}^{\prime} \mathbf{x} \\
\text { subject to } & \mathbf{A x} \geq_{C} \mathbf{b} .
\end{array}
$$

In standard conic problems there are special weight vectors $\mathbf{y}$ that satisfies the subsequent scalar inequality

$$
\langle\mathbf{y}, \mathbf{A x}\rangle \geq\langle\mathbf{y}, \mathbf{b}\rangle
$$

and at the same time, these special vectors preservers the generalized inequality $\mathbf{A x} \geq_{C} \mathbf{b}$ presented in (2-13), for all decision variables $\mathbf{x}$. In the particular case of LP problems, the cone $C$ is the $\mathbb{R}_{+}^{n}$ and the admissible vectors are those with nonnegative elements, which is the same as writing $\mathbf{y} \in C$ or $\mathbf{y} \in \mathbb{R}_{+}^{n}$. However, when the inequality $\geq_{C}$ is associated with a cone $C$ different from the nonnegative orthant, these acceptable vectors are not necessarily the as the one we have seen for LP problems. We are actually looking for a particular set of vectors $\mathbf{y}$ such that

$$
\forall \mathbf{d} \geq_{C} 0: \quad\langle\mathbf{y}, \mathbf{d}\rangle \geq 0,
$$

which are the same vectors from the set

$$
C^{*}=\left\{\mathbf{y} \in \mathbb{R}^{n} \mid \mathbf{y}^{\prime} \mathbf{d} \geq 0, \forall \mathbf{d} \in C\right\} .
$$

The set $C^{*}$ is called dual cone, as the name indicates it is a cone and since $C^{*}$ is a intersection of halfspaces, it is a closed convex set, even when the original cone is not. Also, several other important properties relates $C$ to its dual cone $C^{*}$, some of them are

- If the interior of $C$ is nonempty, then $C^{*}$ is pointed;

- $C_{1} \subseteq C_{2}$, then $C_{2}^{*} \subseteq C_{1}^{*}$;

- If $C$ is a closed convex set, then the interior of $C^{*}$ is nonempty;

- If $C$ is closed and convex set, then the dual cone of $C^{*}$ is the original cone $C$. 
Using the definition of the dual cone, we are set to derive the dual problem of the CP problem (2-13). First, we use the same idea that was developed for the LP problem, whenever $\mathbf{x}$ is a feasible solution for problem (2-13) and $\mathbf{y}$ is a vector in the dual cone, then the scalar inequality is satisfied

$$
\langle\mathbf{y}, \mathbf{A x}\rangle \geq\langle\mathbf{y}, \mathbf{b}\rangle, \text { for all } \mathbf{y} \geq_{C^{*}} 0 \text { and } \mathbf{A x} \geq_{C} \mathbf{b}
$$

As in the LP example, whenever $\mathbf{y}$ is an admissible vector in the dual cone and it also satisfies the following equality expression

$$
\mathbf{A}^{\prime} \mathbf{y}=\mathbf{c}
$$

we have that

$$
\mathbf{c}^{\prime} x=\mathbf{y A}^{\prime} \mathbf{x}=\langle\mathbf{y}, \mathbf{A x}\rangle \geq\langle\mathbf{y}, \mathbf{b}\rangle, \text { for all } \mathbf{y} \geq_{C^{*}} 0,
$$

where for all decision variable $\mathbf{x}$ the $\mathbf{y}^{\prime} \mathbf{b}$ is a lower bound on the optimal value. Hence, the best bound one can achieve for the CP problem is the maximum value of the dual program (2-20)

$$
\begin{array}{cl}
\underset{\mathbf{y}}{\operatorname{maximize}} & \mathbf{y}^{\prime} \mathbf{b} \\
\text { subject to } & \mathbf{A}^{\prime} \mathbf{y}=\mathbf{c} \\
& \mathbf{y} \geq_{C^{*}} 0
\end{array}
$$

Now we are ready to derive the dual of SOCP, which is the case where $C$ is defined as second-order cone.

\subsection{2}

\section{Problems Transformations}

In this section we show some of the techniques for transforming a robust feasible set, which is defined by an infinite number of constraints. Such constraints come from restricting a set of uncertain parameters in a predefined uncertainty set, usually convex and tractable. The result of this transformation is a finite number of constraints that have an equivalent representation of these robust feasible sets. These representations are useful for solving robust optimization problems, however, unfortunately it can only be properly defined for specific combinations of constraints and uncertainty sets. Here we give an illustration of combinations of uncertainty sets and constraints that will be used throughout this dissertation.

First, consider the feasible region restricted by the following linear constraint

$$
\mathcal{X}(\boldsymbol{\eta})=\left\{\mathbf{x}: \mathbf{x}^{\prime} \boldsymbol{\eta} \geq \mathbf{b}\right\}
$$


here $\mathbf{x}$ is the vector decision variables and $\boldsymbol{\eta}$ is the vector uncertainty parameters associated to them. Additionally, assume that the uncertainty parameters are confined in an ellipsoidal uncertainty set

$$
\mathcal{U}_{\boldsymbol{\eta}}=\left\{\boldsymbol{\eta}:(\boldsymbol{\eta}-\hat{\boldsymbol{\eta}})^{\prime} \boldsymbol{\Sigma}_{\eta}^{-1}(\boldsymbol{\eta}-\hat{\boldsymbol{\eta}}) \leq \epsilon^{2}\right\},
$$

where $\boldsymbol{\Sigma}_{\eta}$ is a positive definite matrix, usually taken as the covariance matrix of the uncertainty coefficients and $\epsilon^{2}$ is the tolerance level set by the user. Notice that the size of $\epsilon^{2}$ states the distance (scaled by a positive definite matrix) that the uncertain parameter can deviate from its nominal value $\hat{\boldsymbol{\eta}}$. A useful equivalent representation of this uncertainty set is given by

$$
\mathcal{U}_{\boldsymbol{\eta}}=\left\{\boldsymbol{\eta}:\left\|\boldsymbol{\Sigma}_{\eta}^{-1 / 2}(\boldsymbol{\eta}-\hat{\boldsymbol{\eta}})\right\|_{2} \leq \epsilon\right\},
$$

where $\Sigma_{\eta}^{-1 / 2}$ is lower triangular matrix resulted from the Cholesky factorization of $\Sigma_{\eta}$.

Observe that the feasible set $\left(\mathcal{X}_{\mathbf{R}}\right)$ which satisfies all possible realizations of the uncertainty parameter is defined by a infinite number of linear constraints

$$
\mathcal{X}_{\mathbf{R}}=\bigcap_{\boldsymbol{\eta} \in \mathcal{U}_{\boldsymbol{\eta}}} \mathcal{X}(\boldsymbol{\eta})=\left\{\mathbf{x}: \mathbf{x}^{\prime} \boldsymbol{\eta} \geq \mathbf{b}, \forall \boldsymbol{\eta} \in \mathcal{U}_{\boldsymbol{\eta}}\right\} .
$$

To derive useful transformations for robust optimization problems we illustrate a technique based on conic duality theory. First, we look for the worst-case of the constraint (2-21) over the uncertainty set $\mathcal{U}_{\boldsymbol{\eta}}$, which resumes to the following SOCP problem

$$
\begin{array}{ll}
\underset{\boldsymbol{\eta}}{\operatorname{minimize}} & \mathbf{x}^{\prime} \boldsymbol{\eta} \\
\text { subject to } & \left\|\boldsymbol{\Sigma}_{\eta}^{-1 / 2}(\boldsymbol{\eta}-\hat{\boldsymbol{\eta}})\right\|_{2} \leq \epsilon .
\end{array}
$$

The dual problem of the SOCP (2-24) is defined as

$$
\begin{array}{cl}
\underset{\mathbf{z}, w}{\operatorname{maximize}} & \mathbf{z}^{\prime} \boldsymbol{\Sigma}_{\eta}^{-1 / 2} \hat{\boldsymbol{\eta}}-w \epsilon \\
\text { subject to } & \mathbf{z}^{\prime} \boldsymbol{\Sigma}_{\eta}^{-1 / 2}=\mathbf{x}^{\prime} \\
& \|\mathbf{z}\|_{2} \leq w,
\end{array}
$$

where $w$ and $\mathbf{z}$ are the dual variables. Now, substituting the equality constraint of problem (2-25) on the objective function the dual problem can be rewritten as the following

$$
\begin{array}{ll}
\underset{w}{\operatorname{maximize}} & \mathbf{x}^{\prime} \hat{\boldsymbol{\eta}}-w \epsilon \\
\text { subject to } & \left\|\boldsymbol{\Sigma}_{\eta}^{1 / 2} \mathbf{x}\right\|_{2} \leq w .
\end{array}
$$

Observe that the objective function assumes its maximum value when the dual variable $w$ takes its lowest possible value. Since $w$ is bounded by 
$\left\|\boldsymbol{\Sigma}_{\eta}^{1 / 2} \mathbf{x}\right\|_{2}$, the maximum is achieved when the constraint is satisfied with equality, therefore

$$
w=\left\|\boldsymbol{\Sigma}_{\eta}^{1 / 2} \mathbf{x}\right\|_{2} .
$$

Due to conic strong duality theory the optimal objective function will be the same as the value of the primal problem, thus the minimum value of problem (2-24) for any decision variable $\mathbf{x}$ will be

$$
\mathbf{x}^{\prime} \hat{\boldsymbol{\eta}}-\epsilon\left\|\Sigma_{\eta}^{1 / 2} \mathbf{x}\right\|_{2} .
$$

Replacing (2-28) in the original constraint we obtain the worst-case scenario under the ellipsoidal uncertainty set for the original constraint in terms of the decision variables $\mathbf{x}$. Therefore, the feasible constraint is equivalent to

$$
\mathcal{X}_{\mathbf{R}}=\left\{\mathbf{x}^{\prime} \hat{\boldsymbol{\eta}}-\epsilon\left\|\Sigma_{\eta}^{1 / 2} \mathbf{x}\right\|_{2} \geq b\right\} .
$$

This constraint reformulation is very common in robust optimization and is equivalent to solving the original constraint under the feasible set. These and other useful transformations have been reported by other authors. For instance Ben-Tal and Nemirovski (1998) show that affine constraints combined with uncertainty sets defined by a finite number of second order cone problems can be transformed to a finite number of conic constraints on the original problem. This a general definition of the transformation that was demonstrated here.

Also, Ben-Tal et al. (2002) and Sturm and Zhang (2003) derive a semidefinite programming formulation to quantify the robust feasible sets of quadratic constraints under uncertainty sets defined by a quadratic inequality. Sturm and Zhang (2003) have studied uncertainty sets given by strictly convex or concave quadratic function. Moreover, the complexity to solve problems using ellipsoidal uncertainty sets was studied in Ben-Tal and Nemirovski (1998), where they show that its combination with semidefinite programming constraints are in general NP-hard.

\section{2}

\section{Robust Optimization}

Real optimization problems often have uncertainty parameters. Parameters can be naturally stochastic or uncertain due to errors (e.g., measurement, estimation errors). Preceding to the establishment of robust optimization, data uncertainty problems were often modeled using stochastic optimization. Stochastic optimization assumes that the probability distribution is known or estimated. If it is plausible to assume this condition and the reformulated optimization problem is computationally tractable, then stochastic optimization is 
a possible methodology to solve this problem. For further details in stochastic optimization, see Shapiro et al. (2009) and Birge and Louveaux (1997).

Conversely, robust optimization does not assume that the probabilities distributions are known, instead, it assumes that the parameters uncertainty lies in a predefined uncertainty set. The first idea of uncertainty set was introduced by Soyster (1973), who suggested a linear optimization model in which its optimal value is feasible to all data within a convex set. In exchange for a robust solution to all possible scenarios, this model is known in the literature to produce optimal solutions that are too conservative.

Even though the first published work dates back to the 1970s Soyster (1973), it was many years later that a major development in the theory of robust optimization was taken by Ben-Tal and Nemirovski (1998, 1999), Ghaoui and Lebret (1997) and Ghaoui et al. (1998). Their work provided a detailed analysis on robust optimization framework, in either linear optimization and general convex optimization. To solve the conservativeness issue, Ben-Tal and Nemirovski (1999) introduced a less conservative model, by considering a linear optimization problem with ellipsoidal uncertainties which involved solving a robust counterpart of the nominal problem. They showed that ellipsoidal uncertainty sets resulted in a tractable robust convex problem that could be solved as second-order conic program. Using the concepts of robust optimization, Bertsimas and Sim (2004) provided a new framework to control conservatism of the optimal solution while maintaining the advantages of the linear formulation proposed by Soyster (1973).

Robust optimization reflects the trade-off between robustness and each possible realization of the uncertainty parameter. Since the probability distribution of the parameter is unknown, the general approach is to specify the size and shape of the set around the uncertainty parameter. Where the size of the set determines the probability that the uncertain parameter takes on a value in the set, and the shape dictates the complexity of the optimization problem Fabozzi et al. (2009).

\subsection{1}

\section{Robust Optimization Concepts}

As an example we consider an uncertain linear optimization model, however the discussions that arise from this problem can be extended to other classes of uncertain convex optimization problems. The standard uncertain linear optimization problem takes the following form 


$$
\begin{array}{ll}
\underset{\mathbf{x}}{\operatorname{minimize}} & \mathbf{f}^{\prime} \mathbf{x} \\
\text { subject to } & \mathbf{A x} \leq \mathbf{b}
\end{array}
$$

$$
(\mathbf{A}, \mathbf{b}) \in \mathcal{U} \text {, }
$$

where $\mathbf{x} \in \mathbb{R}^{n}$ are the decision variables, $\mathbf{A} \in \mathbb{R}^{m \times n}, \mathbf{b} \in \mathbb{R}^{m}$ are the uncertain coefficients related to the LO problem and $\mathcal{U}$ is a uncertainty set that is specified by the user. Notice that this problem is equivalent to a collection of LO problems with a common structure that the parameters may varying in a given uncertainty set.

Robust optimization problems that we mention throughout this work are modeled to focus on problems with three main characteristics Nemirovski (2012). First, all decision variables $\mathbf{x} \in \mathbb{R}^{n}$ are "here and now" decisions, meaning that each decision variable must be specified before the uncertainty parameters unfold. The decision maker takes responsibility for the consequences of his decisions when, and only when, the actual data lies in the uncertainty set $\mathcal{U}$ that was previously established. Finally, the decision maker cannot bear violations of the constraints when the data is within the given uncertainty set $\mathcal{U}$, in the literature these type of constraints are known as "hard" constraints Ben-Tal and Nemirovski (1999).

Based on the assumption that the problem must be protected against all uncertainty realizations, we introduce the concept of robust feasibility, that is, the optimization problem should be feasible within all realizations of the uncertainty set. Therefore, a vector $x \in \mathbb{R}^{n}$ is robust feasible if it satisfies the constraints for all realizations of uncertainty, as follows

$$
\mathbf{A x} \leq \mathbf{b} \quad \forall(\mathbf{A}, \mathbf{b}) \in \mathcal{U} .
$$

The idea of robust feasibility naturally leads to these worst-case oriented optimization problems. A central concept around robust optimization methodology is the robust counterpart of an uncertain problem, which is defined as the optimization problem that seeks for the best robust feasible solution over the uncertainty set. The robust counterpart of (2-30) is equivalent to

$$
\begin{array}{cl}
\underset{\mathbf{x}}{\operatorname{minimize}} & \mathbf{f}^{\prime} \mathbf{x} \\
\text { subject to } & \mathbf{A x} \leq \mathbf{b}, \forall(\mathbf{A}, \mathbf{b}) \in \mathcal{U} .
\end{array}
$$

Notice that the robustness with respect to the uncertainty set $\mathcal{U}$ can always be formulated constraint-wise. For some of the examples we may focus on a single constraint, thus for problem (2-32) a constraint-wise uncertainty can be modeled as

$$
(\mathbf{a}+\mathbf{P} \boldsymbol{\eta})^{\prime} \mathbf{x} \leq b, \forall \boldsymbol{\eta} \in \mathcal{U}_{\boldsymbol{\eta}}
$$


where $\boldsymbol{\eta}$ is a constraint-wise realization of the uncertainty set that belongs to the predefined set $\mathcal{U}_{\boldsymbol{\eta}}$. Moreover, in this formulation a robust feasible solution $\mathbf{x} \in \mathbb{R}^{n}$ satisfies all uncertainty constraints $(\mathbf{A}(\boldsymbol{\eta}) \mathbf{x} \leq \mathbf{b})$, for all realizations of $\eta \in \mathcal{U}_{\eta}$.

\subsection{2}

\section{Solving the Robust Counterpart}

Observe that problem (2-32) can be defined as a problem with infinitely many constraints due to the worst case formulation, which makes it intractable in its current form. However, there are robust reformulation techniques to transform it into a one-level optimization problem. Here we describe the details of this approach.

The robust reformation technique is the main procedure in Robust Optimization, which consists of three steps. And as result, we obtain a computationally tractable robust counterpart, which contains a finite number of constraints.

To illustrate the three steps to derive the Robust Counterpart we use a polyhedral uncertainty set:

$$
\mathcal{U}=\{\boldsymbol{\eta}: \mathbf{D} \boldsymbol{\eta}+\mathbf{q} \geq 0\} .
$$

Step 1 (worst case reformulation): Observe that (2-33) can be reformulated in a worst case perspective as

$$
\mathbf{a}^{\prime} \mathbf{x}+\max _{\boldsymbol{\eta} \in \mathcal{U}}\left(\mathbf{P}^{\prime} \boldsymbol{\eta}\right)^{\prime} \mathbf{x} \leq b
$$

Step 2 (duality): In the next step we obtain the dual of the inner maximization problem. Due to strong duality, the dual (minimization problem) is an upper bound of the primal problem (maximization problem) and their optimal value coincides. Therefore, the constraint (2-34) is equivalent to

$$
\mathbf{a}^{\prime} \mathbf{x}+\min _{\mathbf{w}}\left\{\mathbf{q}^{\prime} \mathbf{w}: \mathbf{D}^{\prime} \mathbf{w}=-\mathbf{P}^{\prime} \mathbf{x}, \mathbf{w} \geq 0\right\} \leq b
$$

Step 3 (Robust Counterpart): It is important to mention that the inner minimization problem can be omitted from the constraint. By strong duality, the dual problem is also bounded and feasible, in addition the constraint holds for at least one $\mathbf{w} \in \mathbb{R}^{m}$. Therefore, the final equivalent formulation of the Robust Counterpart (2-32) for this uncertainty set becomes the following

$$
\begin{array}{cl}
\underset{\mathbf{x}, \mathbf{w}}{\operatorname{minimize}} & \mathbf{f}^{\prime} \mathbf{x} \\
\text { subject to } & \mathbf{a}^{\prime} \mathbf{x}+\mathbf{q}^{\prime} \mathbf{w} \leq b \\
& \mathbf{D}^{\prime} \mathbf{w}=-\mathbf{P}^{\prime} \mathbf{x} \\
& \mathbf{w} \geq \mathbf{0},
\end{array}
$$


note that the constraints for (2-36) are linear in $\mathbf{x} \in \mathbb{R}^{n}$ and $\mathbf{w} \in \mathbb{R}^{m}$ and the objective function is also linear, therefore this equivalent problem is tractable. This simple example is just to illustrate this powerful setup to deal with problems that have hard constraints. Moreover, using this same three steps that was described, one can arrive at tractable robust counterparts for different conic uncertainty sets.

\subsection{3}

\section{Defining Uncertainty Sets}

One way of modeling uncertainty is to generate possible outcomes for the uncertain parameters, for instance, one could define a range of values for future asset returns. Optimization under uncertainty is dealt in the robust optimization framework by specifying an uncertainty set, which is a collection of possible scenarios for the uncertain parameters. Moreover, the robust counterpart of the original problem would then contain a set of constraints for each uncertain parameter, and ensure that the original constraint is satisfied for the worst-case scenario under the predefined uncertainty set. Typically the uncertainty sets are chosen such that it satisfies two important properties:

- The robust constraint $\mathbf{a}(\boldsymbol{\eta})^{\prime} \mathbf{x} \leq b \quad \forall \boldsymbol{\eta} \in \mathcal{U}_{\boldsymbol{\eta}}$ is computationally tractable

- For a predefined level of confidence $\xi$, the uncertainty set can be modeled such that the constraints hold with at least a probability $\xi$. This property implicates that for all $\mathbf{x} \in \mathbb{R}^{n}$ and $b \in \mathbb{R}$ the chance constraint holds, therefore

If $\mathbf{a}(\boldsymbol{\eta})^{\prime} \mathbf{x} \leq b \quad \forall \boldsymbol{\eta} \in \mathcal{U}_{\boldsymbol{\eta}}$, then $\mathbf{x}$ also satisfies $\mathbb{P}_{\boldsymbol{\eta}}\left(\mathbf{a}(\boldsymbol{\eta})^{\prime} \mathbf{x} \leq b\right) \geq 1-\xi$.

Usually uncertainty sets that are used in practice range from polytopes to more sophisticated conic-representable sets, that are derived from different assumptions about the uncertainty parameter. For instance, a confidence interval can be defined for an uncertainty parameter, which leads to a polyhedral set known as box uncertainty set. For an uncertainty parameter $\boldsymbol{\eta} \in \mathbb{R}^{n}$, the box uncertainty set is given as follows

$$
\mathcal{U}_{\boldsymbol{\eta}}=\left\{\boldsymbol{\eta}:\left|\eta_{i}-\hat{\eta}_{i}\right| \leq \epsilon_{i}, i=1, \ldots, n\right\},
$$

where $\hat{\boldsymbol{\eta}}$ is the nominal estimated value for $\boldsymbol{\eta}$ and $\boldsymbol{\epsilon}$ denotes the absolute distance difference around the nominal value. This uncertainty set contains the full range of realizations for each uncertainty parameter, therefore it guarantees that each constraint is hardly ever violated $(\xi=0)$. On the other hand, there is a small chance that all uncertain parameters assume their the worst case values at once. The conservativeness of this set led to the development of 
smaller uncertainty sets that still guarantees that each constraint holds in almost every possible scenario.

When additional information, such as moments, symmetry or unimodality about the distributions of uncertainty parameter are available, smaller uncertainty sets can be used. For example, the ellipsoidal uncertainty set proposed by Ben-Tal and Nemirovski (2000) allows to include second moment information on the uncertainty set. Most generally this uncertainty set can be written as the following

$$
\mathcal{U}_{\boldsymbol{\eta}}=\left\{\boldsymbol{\eta}:(\boldsymbol{\eta}-\hat{\boldsymbol{\eta}})^{\prime} \boldsymbol{\Sigma}_{\eta}^{-1}(\boldsymbol{\eta}-\hat{\boldsymbol{\eta}}) \leq \epsilon\right\},
$$

where $\boldsymbol{\Sigma}_{\eta}$ is usually assumed to be the covariance matrix of the parameter $\boldsymbol{\eta}$. The authors have also proved that if $\boldsymbol{\eta}$ are symmetric distributed independent random variables the robust constraint is violated at most with probability $\exp \left(-\epsilon^{2} / 2\right)$. In figure $(2.2)$, we illustrate an example of a two dimensional ellipsoidal uncertainty set.

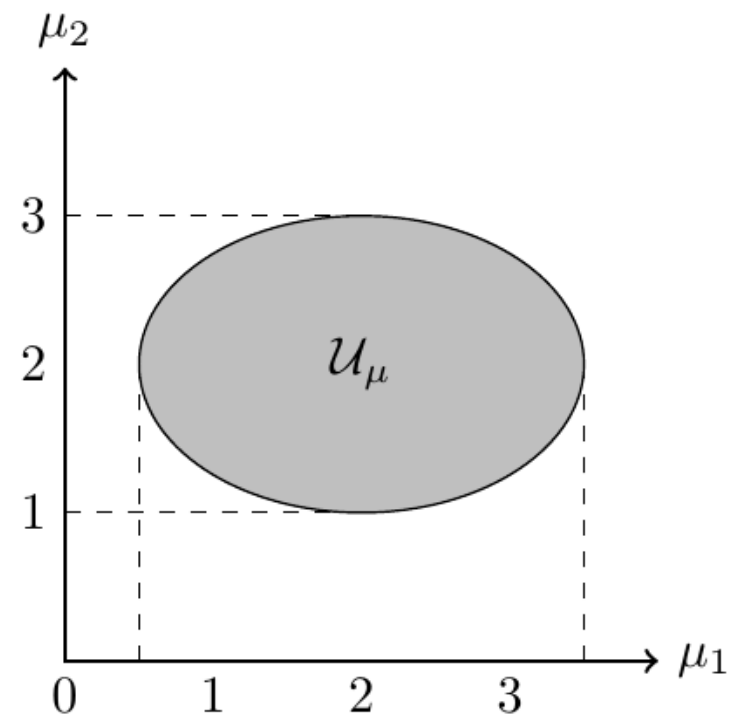

Figure 2.2: Example of an ellipsoidal uncertainty set

A second polyhedron set was proposed by Bertsimas and Sim (2004), they introduced the concept of budgeted uncertainty set. Following the assumption that not all uncertain parameters would go to its worst-case value simultaneously, they introduce a parameter called budget of uncertainty, $\Gamma$, which controls the number of uncertain parameters $(\boldsymbol{\eta})$ that are allowed to deviate from its nominal value. This uncertainty set is given by

$$
\mathcal{U}_{\boldsymbol{\eta}}=\left\{\boldsymbol{\eta}:\left|\eta_{i}-\hat{\eta}_{i}\right| \leq \epsilon_{i} z_{i}, \sum_{i=1}^{n} z_{i} \leq \Gamma, 0 \leq z_{i} \leq 1, i=1, \ldots, n\right\}
$$


here $\boldsymbol{\eta} \in \mathbb{R}^{n}$ and if $\boldsymbol{\eta}$ are independent and symmetrically distributed the confidence level is at most $\exp \left(-\Gamma^{2} /(2 n)\right)$. Note that when $\Gamma=0$ the constraint is equivalent to the constraint in the nominal problem and when assumes the same value as the number of uncertainties we have the box uncertainty set. This is the reason $\Gamma$ is called budget of uncertainty, after all its value exposes the trade off between the nominal problem and the more conservative box uncertainty. It is also important to mention that this uncertainty set leads to a linear programming problem, therefore more tractable than the ellipsoidal uncertainty set.

As an example, we illustrate how the budget of uncertainty affects this uncertainty set and its relation with the box uncertainty set. First, consider a Bertsimas uncertainty set in two dimensions:

$$
\mathcal{U}_{\boldsymbol{\mu}}=\left\{\boldsymbol{\mu}:\left|\mu_{i}-2\right| \leq 1 z_{i}, \sum_{i=1}^{n} z_{i} \leq \Gamma, 0 \leq z_{i} \leq 1, i=1,2\right\} .
$$

In figure 2.3 we project this uncertainty set in a two dimensional space, for $\Gamma=1$ and $\Gamma=2$. Notice that, for $\Gamma=2$, the uncertainty set is equivalent to the uncertainty in equation 2-37, and as the budget of uncertainty becomes smaller the set also reduces. For the specific value of $\Gamma=1$, the set allows one of the parameters to take its nominal value (i.e. average in this example), and the other parameter assumes its worst case value.
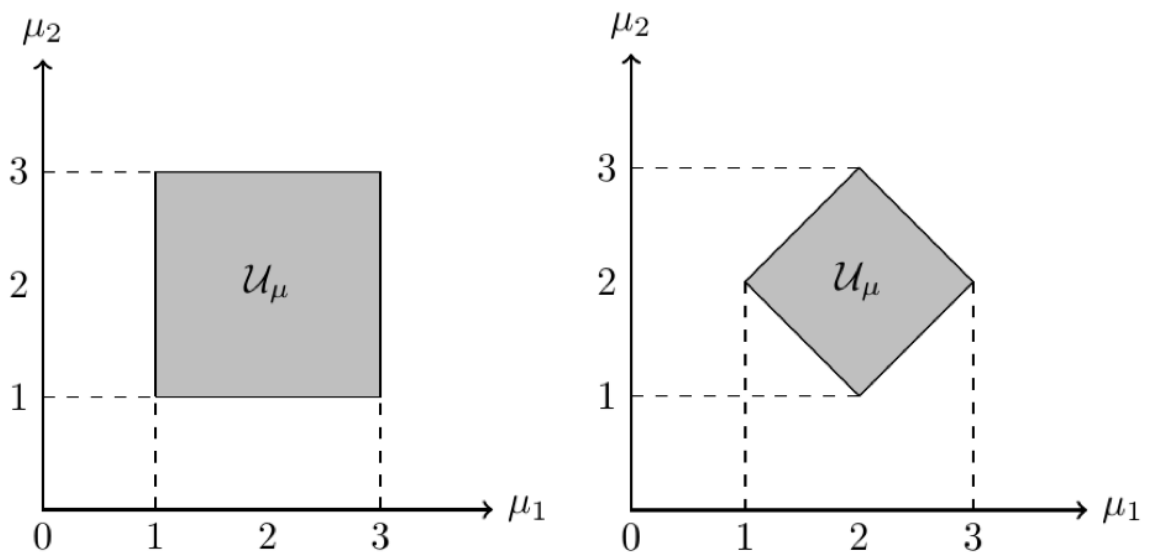

Figure 2.3: Example of Bertsimas uncertainty. On the left: $\Gamma=2$, on the right: $\Gamma=1$.

If regression techniques are used to estimate the uncertainty parameters, polyhedral and ellipsoidal sets comes naturally as potential uncertainty sets, and as was previously mentioned, it can also be associated to probability guarantees for each constraint. 
A newly data driven approach was introduced by Bertsimas et al. (2014). They propose a new methodology that uses data to construct uncertainty sets for robust optimization using hypothesis test. Moreover, on the same article is also provided a thorough guideline with recommendations for practitioners and illustrates applications with portfolio management and queuing.

In recent papers, Bertsimas and Brown (2009) and Natarajan et al. (2009) independently formulated coherent risk measure minimization as robust optimization problem and showed the relation between coherent risk measures and its equivalent uncertainty sets. Moreover, Bertsimas and Takeda (2015) study minimizing a coherent risk measure under a norm equality constraint using a robust optimization framework. To illustrate the correspondence between risk measures and robust optimization uncertainty sets, consider the uncertainty set associated with discrete Conditional Value at Risk (CVaR) generated by a discrete distribution of $\tilde{\boldsymbol{\eta}}$ such that $P\left(\tilde{\boldsymbol{\eta}}=\boldsymbol{\eta}_{i}\right)=p_{i}, i=1, \ldots, n$

$$
\mathcal{U}_{C V a R_{1-\alpha}}=\left\{\sum_{i=1}^{n} z_{i} \boldsymbol{\eta}_{i}: \sum_{i=1}^{n} z_{i}=1, \mathbf{0} \leq \mathbf{z} \leq \frac{1}{\alpha} \mathbf{p}\right\},
$$

In figure 2.4 we illustrate a CVaR uncertainty set of an equiprobable discrete distribution with 20 elements in its sample space (i.e. The set of possible out comes is $\left\{\boldsymbol{\eta}_{1}, \ldots, \boldsymbol{\eta}_{20}\right\}$ and $\left.P\left(\tilde{\boldsymbol{\eta}}=\boldsymbol{\eta}_{i}\right)=\frac{1}{20}, \forall i=1, \ldots, 20\right)$.

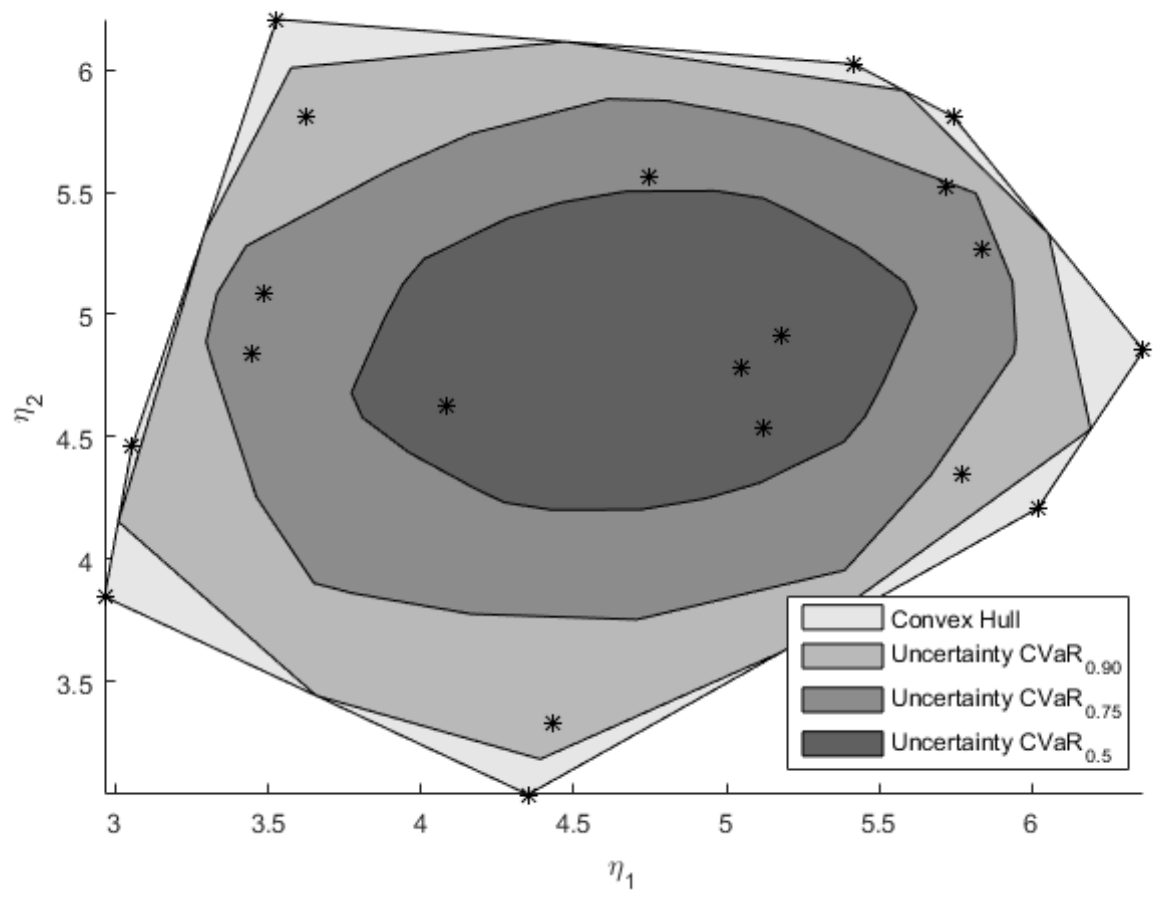

Figure 2.4: Example of CVaR uncertainty set. 
To conclude this section we draw attention to an important misconception regarding the interpretation of the uncertainty set. When an uncertainty set is constructed to include the true parameter with a confidence level of $\xi$, it implicates a stronger probability guarantee than it seems at first. For the reason that, the constraint realization holds at this probability for all realizations of the uncertain parameters outside of the uncertainty set, not only the worst-case scenarios, since it also includes the "good" scenarios. Hence, by solving a problem through a robust optimization perspective the probability guarantee is usually much higher than $1-\xi$.

\subsection{4}

\section{Robust Portfolio Optimization}

Estimated expected returns are likely to diverge from the actual future asset returns, however, we may assume a uncertainty set that can predict the actual future asset return with high probably margin. Hence, for expected returns, uncertainty sets describes a geometric structure around estimated values of future asset returns (Kim et al., 2013). In this dissertation we only consider the case when the covariance matrix of returns is known and the uncertainty relies on the expected returns.

The simplest choice of uncertainty sets for expected returns $(\mu)$ is a box, $\mathcal{U}_{\mu}=\left\{\boldsymbol{\mu}:\left|\mu_{i}-\hat{\mu}_{i}\right| \leq \epsilon_{i}, i=1, \ldots, n\right\}$, where $\epsilon_{i}$ is related to the confidence level around each estimated return. And, the robust portfolio optimization problem is formulated as

$$
\begin{array}{cl}
\underset{\mathbf{x}}{\operatorname{minimize}} & \mathbf{x}^{\prime} \boldsymbol{\Sigma} \mathbf{x} \\
\text { subject to } & \min _{\boldsymbol{\mu} \in \mathcal{U}_{\mu}} \boldsymbol{\mu}^{\prime} \mathbf{x} \geq \mu_{0},
\end{array}
$$

where $\mu_{0}$ is the required expected return from the portfolio. Notice that this is the same uncertainty set proposed by Soyster (1973). Moreover, this model can be reformulated as a one-level optimization problem

$$
\begin{array}{ll}
\underset{\mathbf{x}}{\operatorname{minimize}} & \mathbf{x}^{\prime} \boldsymbol{\Sigma} \mathbf{x} \\
\text { subject to } & \hat{\boldsymbol{\mu}}^{\prime} \mathbf{x}-\boldsymbol{\epsilon}^{\prime}|\mathbf{x}| \geq \mu_{0} .
\end{array}
$$

From problem (2-43) we can derive a intuitive explanation for the singlelevel robust optimization problem. When the weight of an asset $i$ is negative, the robust problem increases its required expected return, $\hat{\mu}+\epsilon_{i}$, on the other hand when it assumes positive values the expected return takes reduction, $\hat{\mu}-\epsilon_{i}$. Fabozzi et al. (2009) interpreted this fact as the risk adjustment by an investor that is averse to estimation error.

Another common structure for the uncertainty set is to consider it an ellipsoidal set, $\mathcal{U}_{\mu}=\left\{\boldsymbol{\mu}:(\boldsymbol{\mu}-\hat{\boldsymbol{\mu}})^{\prime} \boldsymbol{\Sigma}_{\mu}^{-1}(\boldsymbol{\mu}-\hat{\boldsymbol{\mu}}) \leq \epsilon^{2}\right\}$, where $\epsilon^{2}$ is often chosen 
as the quantile of a chi-squared distribution with $\mathrm{n}$ degrees of freedom and $\Sigma_{\mu}$ is the covariance matrix of the estimated expected return. Again, it can be shown by using SOCP duality that problem (2-42) can be formulated as

$$
\begin{array}{ll}
\underset{\mathbf{x}}{\operatorname{minimize}} & \mathbf{x}^{\prime} \mathbf{\Sigma} \mathbf{x} \\
\text { subject to } & \hat{\boldsymbol{\mu}}^{\prime} \mathbf{x}-\epsilon^{2}\left\|\boldsymbol{\Sigma}_{\boldsymbol{\mu}}^{1 / 2} \mathbf{x}\right\|_{2} \geq \mu_{0},
\end{array}
$$

which is a Second Order Cone Programming problem. Ceria and Stubbs (2006) observe that the term $-\epsilon^{2}\left\|\Sigma_{\mu}^{1 / 2} \mathbf{x}\right\|_{2}$ is related to the estimation error and its inclusion in the constraint minimize the effect of estimation error on the optimal decision.

More recently, Fernandes et al. (2016) proposed a new perspective on uncertainty sets for robust portfolio optimization. Their work focused on data-driven polyhedral uncertainty sets constructed with an intuitive loss constraint for asset returns in a rolling horizon scheme. They have also shown empirically that this methodology is able to capture market dynamics and the dependence structure between assets. To illustrate, let's consider a simple return maximization problem subject to an robust loss constraint

$$
\begin{array}{cl}
\underset{\mathbf{x}}{\operatorname{maximize}} & \hat{\boldsymbol{\mu}}^{\prime} \mathbf{x} \\
\text { subject to } & L(\mathbf{r}, \mathbf{x}) \leq \epsilon, \forall \mathbf{r} \in \mathcal{U}_{\mathbf{r}},
\end{array}
$$

where, $\mathbf{r}$ is the unknown vector of asset returns, $\mathbf{x}$ are the decision variables and $\epsilon$ is a scalar that defines the investor's maximum tolerance to a daily loss in his portfolio. Moreover, the loss constraint is defined as

$$
\mathbf{r}^{\prime} \mathbf{x} \geq \gamma, \forall \mathbf{r} \in \mathcal{U}_{\mathbf{r}}
$$

where $\gamma$ is a parameter that denotes the percentage of loss in the portfolio. Moreover, the uncertainty set $\mathcal{U}_{\mathbf{r}}$ is defined as the convex hull of past $n$ observed vectors of daily returns, which can be expressed as

$$
\mathcal{U}_{\mathbf{r}}=\left\{\mathbf{r}: \mathbf{r}=\sum_{t=1}^{n} \mathbf{r}_{t} \xi_{t}, \sum_{t=1}^{n} \xi_{t}=1,0 \leq \boldsymbol{\xi} \leq 1,\right\},
$$

here, $\mathbf{r}_{t}$ are $n$ sample historical returns.

The authors have shown that to guarantee robust feasibility of the loss constraint for any optimal decision $\mathbf{x}$ it is sufficient to include $n$ linear constraints (2-46) for each return sample $\mathbf{r}_{t}$. Therefore, problem (2-45) can be formulated in this framework as

$$
\begin{array}{ll}
\underset{\mathbf{x}}{\operatorname{maximize}} & \hat{\boldsymbol{\mu}}^{\prime} \mathbf{x} \\
\text { subject to } & \mathbf{r}_{t}^{\prime} \mathbf{x} \geq \gamma, \forall \mathbf{r}_{t}=1, \ldots, n,
\end{array}
$$

This approach enables the investor to adaptively generate polyhedral uncertainty sets that changes over time according to market dynamics. In 
figure 2.5 we illustrate this uncertainty set in different days using a sample of 252 daily returns observations. We can clearly see that the uncertainty set expands when the market is more volatile, as well is captures the negative correlation between both assets.
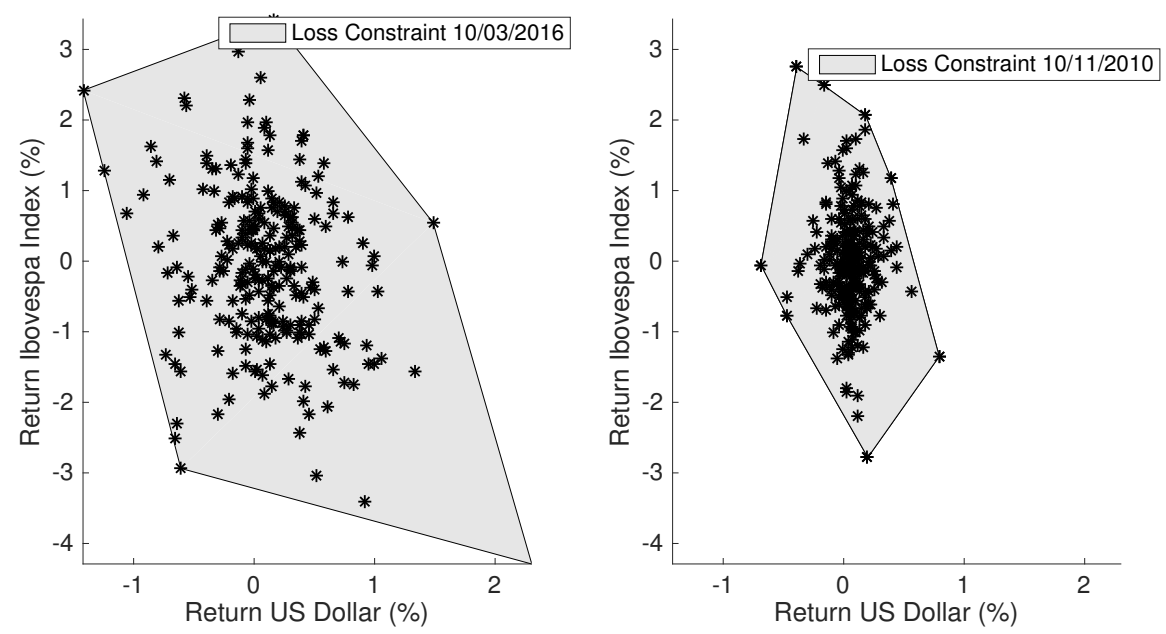

Figure 2.5: Example of robust loss constraint in different market conditions.

\section{3}

\section{Black-Litterman Model}

The Black-Litterman method (Black and Litterman, 1990) was created to be a practical and more stable portfolio management method. The portfolio is created to provide intuitive weights to for the investors that can be adjusted according to their opinions about the market. The methodology starts by defining a neutral market portfolio and views determined by the user, then these parameters are combined to construct a new updated market distribution. The optimal porfolio is achieved by using this new distribution as input to the classical mean-variance portfolio optimization problem.

This section reviews the Black-Litterman model, proposed by Black and Litterman (1990) and Black and Litterman (1992), for more information on the topic we also refer to Walters (2009), Meucci (2008) and Idzorek (2007). It is also presented here a extension of the Black-Litterman model done by Meucci (2008). 


\subsection{1}

\section{The Model}

Consider a market of $\mathrm{N}$ risky securities or asset classes where all investors maximize their portfolio return for a given limit of risk. That is, investors look to solve the classical Markowitz's portfolio optimization problem:

$$
\begin{array}{ll}
\underset{\mathbf{x}}{\operatorname{maximize}} & \boldsymbol{\mu}^{\prime} \mathbf{x} \\
\text { subject to } & \mathbf{x}^{\prime} \boldsymbol{\Sigma} \mathbf{x} \leq \sigma_{0}^{2},
\end{array}
$$

where $\boldsymbol{\Sigma}$ is the covariance matrix of asset returns, $\mathbf{x}$ is the amount of wealth invested on each security, $\boldsymbol{\mu}$ is the expected asset excess returns and $\sigma_{0}^{2}$ is the risk limit specified by the investor.

A common path that is taken to solve equation (2-49), is to estimate the covariance matrix and asset returns from an econometric model. However, finding a stable estimation is rather a difficult task. With that in mind, Black and Litterman (1990) suggested a framework that combines two set of inputs, the market equilibrium and investor's views.

\section{Market Equilibrium Model}

We start by considering a market with $n$ risky assets, where the returns follows a multivariate normal distribution:

$$
\mathbf{r} \sim N(\boldsymbol{\mu}, \boldsymbol{\Sigma}),
$$

where $\boldsymbol{\mu} \in \mathbb{R}^{n}$ is the expected return and $\boldsymbol{\Sigma} \in \mathbb{R}^{n \times n}$ is the covariance matrix, which is considered to be known and estimated from historical data.

The Black Litterman model assumes that distributions of asset returns are consistent with the market equilibrium. Hence, if all investors solve equation (2-49) there exists $\delta$, such that we can solve explicitly this problem and obtain the relationship between market equilibrium portfolio $\left(\mathbf{x}_{m k t}\right)$ and the reference expected returns $(\boldsymbol{\mu})$

$$
\boldsymbol{\mu}=2 \delta \boldsymbol{\Sigma} \mathbf{x}_{m k t}
$$

Now, multiplying (2-51) by $\mathbf{x}_{m k t}, \delta=\mathbf{x}_{m k t}^{\prime} \boldsymbol{\mu} /\left(2 \mathbf{x}_{m k t}^{\prime} \Sigma \mathbf{x}_{m k t}\right)$. This parameter is known in the literature as risk aversion level, as it measures the risk-return trade-off of the portfolio. Thus, we define a market price of risk, which for an unobservable value, $\sigma_{m k t}^{2}$, we have that the market allocation, $\mathbf{x}_{m k t}$, satisfies the optimal value of 2-49 and

$$
\hat{\delta}=\frac{\mathbf{x}_{m k t}^{\prime} \boldsymbol{\mu}}{2 \sigma_{m k t}^{2}}
$$


where $\hat{\delta}$ is the average portfolio risk aversion. The magnitude of $\hat{\delta}$ reflects investor's aversion to estimation risk. When $\hat{\delta}$ is small, the investor's aversion to risk is also small, which leads to more risky portfolios. From an optimization perspective, it happens because the portfolio variance is not penalized as much in the objective function. The Black-Litterman model aims to find the average risk aversion parameter of a given reference portfolio. Although there are multiple studies on $\hat{\delta}$, which affects directly the market equilibrium returns, there is no consensus on how to estimate $\hat{\delta}$. Moreover, these results are centered around the capital asset price model equilibrium (CAPM). For the classical proofs of these equations and further results on CAPM theory see Elton et al. (2009) and Sharpe (1964).

It is a common practice to calibrate $\hat{\delta}$ so that the portfolio can better represent the risk-return characteristics that is desired. Pachamanova and Fabozzi (2011) recommends to calibrate via backtests using the historical data. Furthermore, other authors specified the value of $\hat{\delta}$ that they have chosen. For instance, Bevan and Winkelmann (1998) calibrate the market equilibrium returns to an average target Sharpe Ratio based on their past experience, in their global fixed income example they used a Sharpe Ratio of 1.0. Black and Litterman (1992) used a Sharpe Ratio approximately 0.5 in the example shown in their paper. Allaj (2013) proposed a econometric methodology to estimate the risk averse parameter for the Black-Litterman framework. In practice, there is no consensus on how this parameter should be estimated.

The Black-Litterman model considers the true expected returns $\boldsymbol{\mu}$ of the securities are unknown and assumes that the CAPM serves as a reasonable estimate for the expected returns, as a result the equilibrium model is defined as

$$
\boldsymbol{\pi}=2 \hat{\delta} \boldsymbol{\Sigma} \mathbf{x}_{m k t}+\boldsymbol{\epsilon}_{m}, \boldsymbol{\epsilon}_{m} \sim N(\mathbf{0}, \tau \boldsymbol{\Sigma}),
$$

here $\tau \boldsymbol{\Sigma}$ represents the confidence on the equilibrium expected return model. For instance, a small value of $\tau$ implies a low confidence in our market equilibrium estimate. On the other hand a high value indicates a high confidence. As a result, the model states that $\boldsymbol{\mu}$ is normally distributed

$$
\boldsymbol{\mu} \sim N(\boldsymbol{\pi}, \tau \boldsymbol{\Sigma}),
$$

The parameter $\tau$ was proposed to deal with market equilibrium uncertainties, which is a scaling factor for the uncertainty of the estimated mean return (see He and Litterman (1999); Meucci (2008)). This parameter is considered one of the most confusing aspects of the Black-Litterman model. The original model presented in Black and Litterman (1992) does not specify how 
to estimate it. Despite that, in the literature there are several methodologies to estimate $\tau$. The first methodology provides an idea of the magnitude of $\tau$ by replacing it with $\frac{1}{T}$, where $T$ is the number of observations. However, this methodology is not well-founded, it is simply provides an idea of the magnitude of $\tau$. Moreover, Satchell and Scowcroft (2000) treats $\tau$ as a random variable and others scholars give only recommended values of $\tau$. Many of these authors argues that $\tau$ is greater than zero and smaller than one.

\subsection{2}

\section{Specifying Investor's Views}

An investor view is an information or opinion on the market that possibly diverges from the reference market model. Black Litterman model considers these views as expectations, $q_{1}, q_{2}, \ldots, q_{m}$, on different portfolios, $\mathbf{p}^{1}, \mathbf{p}^{2}, \ldots, \mathbf{p}^{m}$, which is represented as the matrix $\mathbf{P} \in \mathbb{R}^{m \times n}$. In the normal market these views corresponds to statements on the expected asset returns $\boldsymbol{\mu}$ Meucci (2008). Formally, the Black-Litterman model expresses the views as

$$
\mathbf{q}=\mathbf{P} \boldsymbol{\mu}+\boldsymbol{\epsilon}_{v}, \boldsymbol{\epsilon}_{v} \sim N(\mathbf{0}, \boldsymbol{\Omega}),
$$

where $\Omega$ is the covariance matrix of the views extimation error, which, in a sense, expresses the confidence of the investor on the views, $\mathbf{P}$ is the matrix of the portfolios which the investor has a view and and $\mathbf{q}$ states the expected return of each portfolio view. Originally the covariance matrix $\Omega$ can be expressed in two different ways, which is differentiated by the dependence between views.

- In the case of independent views, the matrix $\Omega$ is chosen in such a way that the off diagonal elements should be equal to zero, therefore

$$
\boldsymbol{\Omega}=\operatorname{diag}\left(\tau_{1}, \ldots, \tau_{n}\right)
$$

- In the case where there is dependence between each view, Meucci (2008) suggested to use the same dependent structure expressed by the estimated covariance matrix, modified by the portfolio matrix $\mathbf{P}$ to match the dimension of the original views $\mathbf{q}$.

$$
\boldsymbol{\Omega}=\frac{1}{\tau_{0}} \mathbf{P} \mathbf{\Sigma} \mathbf{P}^{\prime}
$$

Where, $\tau_{0} \in(0, \infty)$ represents the confidence on the views. When $\lim _{\tau_{0} \rightarrow 0} \tau_{0}$ it neglects the market and only consider the views and with $\lim _{\tau_{0} \rightarrow \infty} \tau_{0}$ expresses full confidence on the CAPM model. 


\subsection{3 \\ Market Distribution Update}

After the market equilibrium and investor's views are specified, we proceed to update the returns distributions. There are two equivalent approaches that can be used to arrive at the Black-Litterman formulation, which is known as the posterior distribution. Here we follow the derivation shown at Fabozzi et al. (2007) which based on a standard econometrical technique, known as mixed estimation technique described by Theil (1971). First, we combine the investor views and market equilibrium equations in a standard linear model for the expected returns

$$
\mathbf{y}=\mathbf{X} \boldsymbol{\mu}+\boldsymbol{\epsilon}, \boldsymbol{\epsilon} \sim N(\mathbf{0}, \mathbf{W}),
$$

with each respective term being

$$
\mathbf{y}=\left[\begin{array}{l}
\boldsymbol{\pi} \\
\mathbf{q}
\end{array}\right], \mathbf{X}=\left[\begin{array}{l}
\mathbf{I}_{n} \\
\mathbf{P}
\end{array}\right], \mathbf{W}=\left[\begin{array}{cc}
\tau \boldsymbol{\Sigma} & 0 \\
0 & \Omega
\end{array}\right],
$$

where $\mathbf{I}_{n}$ is an identity matrix of the same dimension as the number of assets. From the following optimization problem we calculate the Generalized Least Squares (GLS) estimatior for $\boldsymbol{\mu}$

$$
\underset{\boldsymbol{\mu}}{\operatorname{minimize}}\left\|\mathbf{W}^{-1}(\mathbf{y}-\mathbf{X} \boldsymbol{\mu})\right\|_{2}^{2}
$$

From the solution of the optimization problem (2-60) we obtain the estimated expected return of the Black-Litterman model, where $\hat{\boldsymbol{\mu}}_{B L}=$ $\left(\mathbf{X}^{\prime} \mathbf{W}^{-1} \mathbf{X}\right)^{-1} \mathbf{X}^{\prime} \mathbf{W}^{-1} \mathbf{y}$. Applying this result to the original values of $\mathbf{y}, \mathbf{X}$ and $\mathrm{W}$ we arrive at

$$
\hat{\boldsymbol{\mu}}_{B L}=\left[(\tau \boldsymbol{\Sigma})^{-1}+\mathbf{P}^{\prime} \boldsymbol{\Omega}^{-1} \mathbf{P}\right]^{-1}\left[(\tau \boldsymbol{\Sigma})^{-1} \boldsymbol{\pi}+\mathbf{P}^{\prime} \mathbf{\Omega}^{-1} \mathbf{q}\right] .
$$

And, the variance estimated by the Bayesian update is given by

$$
\Sigma_{B L}^{\mu}=\left[(\tau \boldsymbol{\Sigma})^{-1}+\mathbf{P}^{\prime} \mathbf{\Omega}^{-1} \mathbf{P}\right]^{-1}
$$

However we are interested in the posterior distribution of the risky securities, not the posterior distribution of the mean estimate. To find this distribution we can equivalently rewrite (2-50) as $\mathbf{r} \stackrel{d}{=} \boldsymbol{\mu}+\boldsymbol{\epsilon}_{r}$, where $\boldsymbol{\epsilon}_{r} \sim$ $N(\mathbf{0}, \boldsymbol{\Sigma})$. Hence, assuming that $\boldsymbol{\mu}$ and $\boldsymbol{\epsilon}_{r}$ are independent, the posterior covariance matrix of reference model is

$$
\boldsymbol{\Sigma}_{B L}=\boldsymbol{\Sigma}+\left[(\tau \boldsymbol{\Sigma})^{-1}+\mathbf{P}^{\prime} \mathbf{\Omega}^{-1} \mathbf{P}\right]^{-1}
$$

From equation (2-61) we see that the Black-Litterman expected return is a weighted linear combination of market equilibrium $\boldsymbol{\pi}$ and the investor's views q. As we will later show, our approach uses this fact to develop robust formulations of the Black-Litterman model under conflicting views 
$\mathbf{q} \in \mathcal{Q}_{q}$, where $\mathcal{Q}_{q}$ is the uncertainty set of the views created from multiple forecasters. In addition, one could also extend our models to an uncertain market equilibrium $\boldsymbol{\pi} \in \mathcal{P}_{\pi}$, in the same manner, $\mathcal{P}_{\pi}$ is the uncertainty set of the market equilibrium.

Example 1 To ilustrate, we consider a simplified case of a Brazilian hedge fund that invests in four asset classes: Brazilian fixed income bonds, Brazilian inflation-linked bonds, U.S. Dollar (BRLUS) and Brazilian stock market index (IBOV). To represent the historical daily return series of the fixed income securities it is used ANBIMA fixed income index IRF-M and ANBIMA inflation-linked box index IMA-B. We consider a data set starting from October '16 and use 12 months of past daily returns observations to estimate de covariance matrix.

The estimated historical variances (annualized) on the asset classes mentioned are approximately $\boldsymbol{\sigma} \approx\left[\begin{array}{llll}3.5 \% & 7.1 \% & 18.1 \% & 26.9 \%\end{array}\right]^{\prime}$ and the correlation matrix

$$
\boldsymbol{C}=\left[\begin{array}{cccc}
1 & 0.84 & -0.27 & 0.51 \\
& 1 & -0.28 & 0.49 \\
& & 1 & -0.42 \\
& & & 1
\end{array}\right]
$$

To determine the reference model excess mean returns $\boldsymbol{\pi}$ we start from a hypothetical market portfolio weights $\mathbf{x}_{m k t}=\left[\begin{array}{lllll}20 \% & 26 \% & 17 \% & 37 \%\end{array}\right]^{\prime}$. Next, we follow He and Litterman (1999) and exogenously define $\hat{\delta}=1.25$. Now, from (2-53) we obtain

$$
\boldsymbol{\pi}=\left[\begin{array}{llll}
0.6 \% & 1.1 \% & -0.8 \% & 6.4 \%
\end{array}\right]^{\prime}
$$

We have taken $\tau=1 / 252$, corresponding to using 252 historical observations and in equation (2-57) we set $\tau_{0}=2 / \tau$, representing a conviction 2 times stronger in views compared to the equilibrium estimate model. Also, we assume that the investor has two views, the first view is an absolute view and the second one is a relative view:

1. Inflation-indexed bonds will have an excess return of $1.5 \%$.

2. U.S Dollar will outperform Brazilian stock market index by $1 \%$.

Mathematically, these views are expressed as 


$$
\left[\begin{array}{c}
1.5 \% \\
1 \%
\end{array}\right]=\left[\begin{array}{cccc}
0 & 1 & 0 & 0 \\
0 & 0 & 1 & -1
\end{array}\right]\left[\begin{array}{c}
\mu_{I R F-M} \\
\mu_{I M A-B} \\
\mu_{D O L} \\
\mu_{I B O V}
\end{array}\right]+\left[\begin{array}{l}
\epsilon_{1} \\
\epsilon_{2}
\end{array}\right] .
$$

Moreover, using the Black-Littermaan framework the excess return vector becomes

$$
\boldsymbol{\mu}_{B L}=\left[\begin{array}{llll}
0.6 \% & 1.3 \% & 1.4 \% & 3.17 \%
\end{array}\right]^{\prime} .
$$

With the updated distribution, it is now possible to solve Markowitz problem (2-49), possibly under a set of additional convex constraints, such as boundaries on asset classes, turnover, or risk exposure constraint. This secondorder cone programming problem can be easily solved numerically. In this example, in our portfolio optimization model we assume no short sales and leverage allowed, i.e. $\mathbf{x} \geq 0$ and $\mathbf{x}^{\prime} \mathbf{1}=1$.

In figure 2.6 we plot the optimal allocation for the reference model and the posterior model. Consistently with the views, the exposure to US Dollar increases to lower risk levels of risk aversion, also the exposure to inflationindexed bonds increases across all levels of risk aversion whereas at the same time the exposure to fixed income bonds and equity index decreases for the same levels of volatility. 

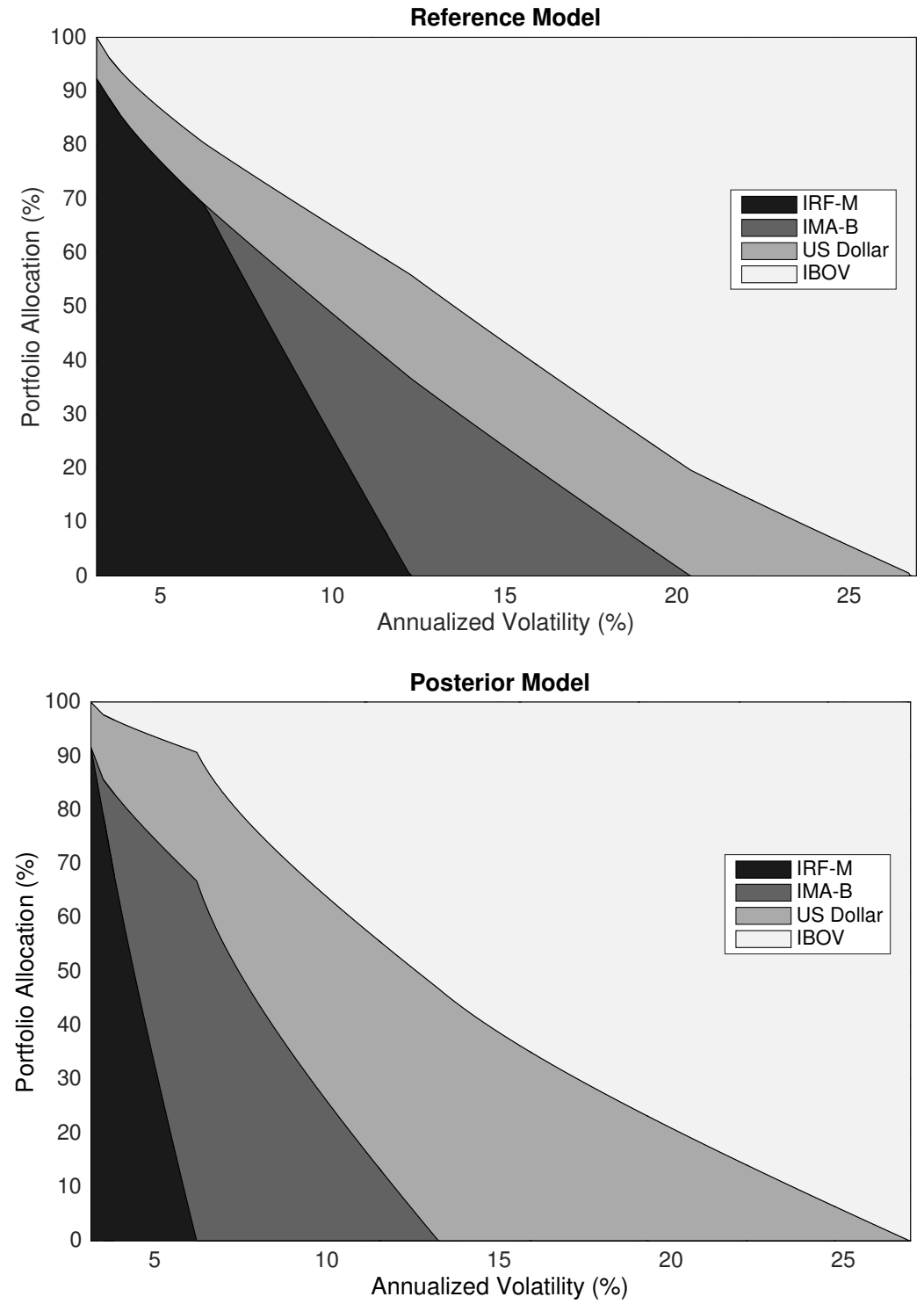

Figure 2.6: Black-Litterman allocation model with and without views

\subsection{4}

\section{Black-Litterman Model Extensions: View on Risk Factors}

Meucci (2008) proposed minor modifications to the original model, which can improve its range of applications. Meucci proposes a model that expresses the views on the market $\mathbf{r}$, not directly on the expected return $\boldsymbol{\mu}$.

On the extreme condition when $\Omega \rightarrow \infty$, intuitively one would presume that the estimated distribution would be equal to the market reference model (2-50). However, in the Black-Litterman model under this condition the estimated distribution becomes

$$
\mathbf{r}_{B L} \sim N(\boldsymbol{\pi},(1+\tau) \boldsymbol{\Sigma})
$$


Therefore, for a specific reference market model the posterior distribution is distorted, unless $\tau=0$, which might go against the user's intuition. To solve this problem Meucci (2008) uses the same framework of the original BlackLitterman model. However, he assumes that the expected return $\boldsymbol{\mu}$ is not a random variable, therefore $\tau=0$ and $\boldsymbol{\mu}=\boldsymbol{\pi}$. In addition, the investor has views on linear functions of the market $\mathbf{q}=\mathbf{P r}$, where $\mathbf{P}$ is the portfolio matrix as in (2-55). As in the original Black-Litterman, the views are assumed to be normal random variables

$$
\mathbf{q}=\operatorname{Pr}+\boldsymbol{\epsilon}_{v}, \boldsymbol{\epsilon}_{v} \sim N(\mathbf{0}, \boldsymbol{\Omega}),
$$

where $\Omega$ represents the uncertainty related to the investor's views on the market reference model, as in (2-55). Moreover, applying the same methodology showed for original model, we obtain an similar market posterior distribution, given as follows

$$
\mathbf{r} \mid \mathbf{q} ; \boldsymbol{\Omega} \sim N\left(\boldsymbol{\mu}_{B L}^{\mathbf{r}}, \boldsymbol{\Sigma}_{B L}^{\mathbf{r}}\right),
$$

where, the mean and covariance matrix are

$$
\begin{aligned}
& \boldsymbol{\mu}_{B L}^{\mathbf{r}}=\left[(\boldsymbol{\Sigma})^{-1}+\mathbf{P}^{\prime} \boldsymbol{\Omega}^{-1} \mathbf{P}\right]^{-1}\left[(\boldsymbol{\Sigma})^{-1} \boldsymbol{\pi}+\mathbf{P}^{\prime} \boldsymbol{\Omega}^{-1} \mathbf{q}\right] \\
& \boldsymbol{\Sigma}_{B L}^{\mathbf{r}}=\left[(\boldsymbol{\Sigma})^{-1}+\mathbf{P}^{\prime} \boldsymbol{\Omega}^{-1} \mathbf{P}\right]^{-1}
\end{aligned}
$$

These extension proposed by Meucci is very similar to the original Black-Litterman model. However, there are a couple advantages of using this formulation. First, parameter $\tau$ is never used here, which eliminates part of the subjectivity underling the original model. Second, in the market posterior model there is no need to include the original covariance matrix as in (2-63). In addition, under extreme confidence level (i.e. $\Omega \rightarrow \infty$ ) these formulation results in a model that is consistent with the market reference. In other words, the posterior distribution would be the same as the prior distribution. 


\section{Proposed Robust Model based on Black-Litterman Approach}

In this chapter we propose robust models based on the Black-Litterman framework, where the investor incorporates conflicting views on the same asset and sets a confidence region for the market equilibrium. We propose models that construct uncertainty sets on the views with complete and incomplete information. To model uncertainty, we adapt the uncertainty sets presented in (2.2.3) to different possible scenarios from an investor's perspective. We divide this chapter in three sections: in the first section we present our general robust model based on Black-Litterman approach, in the second one we propose an uncertainty set based on complete information on the views from the forecasters, therefore the investor is perfectly informed of all portfolio views from the forecasters, before the realization of future asset returns. Whereas in the last part of this chapter we focus on uncertainty sets constructed with partial information about the views, which is assumed that the decision maker has some statistical information about the forecasters.

\section{1}

\section{General Robust Black-Litterman Model}

We have seen in section 2.3 that the Black-Litterman model expected return is a linear combination of the market reference model and the expected return implied by the views, this result is shown in equation (2-61). The linear combination are given by the following matrices

$$
\begin{aligned}
& \mathbf{A}=\left[(\tau \boldsymbol{\Sigma})^{-1}+\mathbf{P}^{\prime} \boldsymbol{\Omega}^{-1} \mathbf{P}\right]^{-1}(\tau \boldsymbol{\Sigma})^{-1} \\
& \mathbf{B}=\left[(\tau \boldsymbol{\Sigma})^{-1}+\mathbf{P}^{\prime} \boldsymbol{\Omega}^{-1} \mathbf{P}\right]^{-1}\left[\mathbf{P}^{\prime} \boldsymbol{\Omega}^{-1}\right],
\end{aligned}
$$

and, for $n$ assets we have that $\mathbf{A}+\mathbf{B P}=\mathbf{I}$, where $\mathbf{I} \in \mathbb{R}^{n \times n}$ is an identity matrix. Following this line of thought, we start by defining our proposed general mean-variance robust Black-Litterman framework

$$
\begin{array}{cl}
\underset{\mathbf{x}}{\operatorname{minimize}} & \mathbf{x}^{\prime} \boldsymbol{\Sigma}_{B L} \mathbf{x} \\
\text { subject to } & \boldsymbol{\mu}_{B L}(\boldsymbol{\pi}, \mathbf{q})^{\prime} \mathbf{x} \geq \mu_{0}, \forall \boldsymbol{\pi} \in \mathcal{P}_{\pi}, \mathbf{q} \in \mathcal{Q}_{q}
\end{array}
$$

where $\mu_{0} \in \mathbb{R}$ is the expected return constraint and $\boldsymbol{\mu}_{B L}(\boldsymbol{\pi}, \mathbf{q}) \in \mathbb{R}^{n}$ is the return implied by the Black-Litterman model, defined as $\boldsymbol{\mu}_{B L}(\boldsymbol{\pi}, \mathbf{q})=\mathbf{A} \boldsymbol{\pi}+\mathbf{B q}$ and $\mathbf{q}$ and $\boldsymbol{\pi}$ belongs uncertainty sets. This general model considers a possible robustness on both the market equilibrium and investor's opinion. To deal only 
with conflicting views in a robust optimization framework, we rewrite (3-1) as the following

$$
\begin{array}{cl}
\underset{\mathbf{x}}{\operatorname{minimize}} & \mathbf{x}^{\prime} \boldsymbol{\Sigma}_{B L} \mathbf{x} \\
\text { subject to } & (\mathbf{a}+\mathbf{B q})^{\prime} \mathbf{x} \geq \mu_{0}, \forall \mathbf{q} \in \mathcal{Q}_{q},
\end{array}
$$

where $\mathbf{a}=\mathbf{A} \boldsymbol{\pi}$ (i.e. the market equilibrium is defined as a point-wise estimate of CAPM) and $\mathcal{Q}_{q}$ is the uncertainty set defined by the user. Here we interpret $\mathcal{Q}_{q}$ as an uncertainty set of conflicting views on the same universe of asset classes.

In problem (3-2), observe that $\boldsymbol{\mu}_{B L}$ is an affine function of the views $\mathbf{q}$ and the expected return constraint is linear in both the decision variables $\mathbf{x}$ and the uncertainty parameter $\mathbf{q}$. Moreover, assuming that $\mathcal{Q}_{q}$ is a compact convex set allows to derive a tractable robust formulation applying the three steps described in section 2.2.2. In the next sections, we propose three models that explore uncertainty sets regarding the views $\mathbf{q}$ under this framework. For each model, we also try to give some intuition and how they would fit for practical use.

\section{2}

\section{Black-Litterman with Multiple Forecasters}

In the first model, suppose that the portfolio manager receives $n$ views from $f$ different analysts $, \mathbf{q}_{1}, \mathbf{q}_{2}, \ldots, \mathbf{q}_{f}$ for the same portfolio $\mathbf{P} \in \mathbb{R}^{m \times n}$, on different assets and each one of these views has to satisfy the robust problem (3-2). Collectively the uncertainty set can be represented as a convex hull of the analysts views

$$
\mathcal{Q}_{q}=\left\{\mathbf{q} \in \mathbb{R}^{n} \mid \mathbf{q}=\theta_{1} \mathbf{q}_{1}+\cdots+\theta_{f} \mathbf{q}_{f}, \boldsymbol{\theta} \in \Delta_{p}\right\},
$$

where $\Delta_{p}$ describes the probability simplex, which is given by

$$
\Delta_{p}=\left\{\boldsymbol{\theta} \in \mathbb{R}^{f} \mid \boldsymbol{\theta} \geq \mathbf{0}, \boldsymbol{\theta}^{\prime} \mathbf{1}=1\right\} .
$$

Notice that $\mathcal{Q}_{q}$ is a polyhedron set, and it can also be expressed by a set of linear equalities and inequalities. Thus, under this uncertainty set we can write the robust constraint of problem (3-2) as

$$
\sum_{i=1}^{f} \theta_{i}\left(\mathbf{a}+\mathbf{B q}_{i}\right)^{\prime} \mathbf{x} \geq \mu_{0}, \quad \forall \boldsymbol{\theta} \in \Delta_{p}
$$

hence, in the worst case perspective, constraint (3-5) can be formulated as

$$
\min _{\boldsymbol{\theta} \in \Delta_{p}}\left\{\sum_{t=1}^{f} \theta_{i}\left(\mathbf{a}+\mathbf{B q}_{i}\right)^{\prime} \mathbf{x}\right\} \geq \mu_{0} .
$$


To guarantee that (3-6) is satisfied for all forecasters and for any allocation $\mathbf{x}$, it is enough to include $f$ constraints which will bound the robust constraint to the convex hull denoted by uncertainty set $\mathcal{Q}_{q}$.

Proof. First, consider inner optimization problem of (3-6)

$$
\begin{array}{cl}
\underset{\boldsymbol{\theta}}{\operatorname{minimize}} & \sum_{t=1}^{f} \theta_{i}\left(\mathbf{a}+\mathbf{B q}_{i}\right)^{\prime} \mathbf{x} \\
\text { subject to } & \boldsymbol{\theta}^{\prime} \mathbf{1}=1: \phi \\
& \boldsymbol{\theta} \geq \mathbf{0},
\end{array}
$$

where $\phi$ is a dual variable. Then, the dual problem of (3-7) corresponds to the following LP

$$
\begin{array}{ll}
\underset{\phi}{\operatorname{maximize}} & \phi \\
\text { subject to } & \phi \leq\left(\mathbf{a}+\mathbf{B q}_{i}\right)^{\prime} \mathbf{x}, \forall i=1, \ldots, f,
\end{array}
$$

which yields to an equivalent robust constraint

$$
\max _{\phi}\left\{\phi \mid \phi \leq\left(\mathbf{a}+\mathbf{B} \mathbf{q}_{i}\right)^{\prime} \mathbf{x}, \forall i=1, \ldots, f,\right\} \geq \mu_{0} .
$$

Therefore, following step 3 of section (2.2.2), we can omit the inner maximization problem. In addition, we apply Fourier-Motzkin scheme to generate an equivalent set of constraints and eliminate the dual variable $\phi$ from the robust constraint. Hence, the final formulation of the robust counterpart becomes

$$
\left(\mathbf{a}+\mathbf{B} \mathbf{q}_{i}\right)^{\prime} \mathbf{x} \geq \mu_{0}, \forall i=1, \ldots, f .
$$

Now, we can rewrite the original optimization problem (3-1) as a singlelevel equivalent problem

$$
\begin{array}{cl}
\underset{\mathbf{x}}{\operatorname{minimize}} & \mathbf{x}^{\prime} \boldsymbol{\Sigma}_{B L} \mathbf{x} \\
\text { subject to } & \hat{\boldsymbol{\mu}}_{B L, i}^{\prime} \mathbf{x} \geq \mu_{0}, \forall i=1, \ldots, f
\end{array}
$$

where $\hat{\boldsymbol{\mu}}_{B L, i}=\mathbf{a}+\mathbf{B q}_{i}$. Notice, in problem (3-11) the optimal value satisfies the return constraint for all forecasters.

Example 2 We illustrate this model using the same Brazilian hedge fund case study presented in Example (1). However, now let's consider that the Brazilian fund wants to add one conflicting view to the Black-Litterman framework from a different analyst. Let's recall the views and the Black-Litterman excess return vector

$$
\left[\begin{array}{c}
1.5 \% \\
1 \%
\end{array}\right]=\left[\begin{array}{cccc}
0 & 1 & 0 & 0 \\
0 & 0 & 1 & -1
\end{array}\right]\left[\begin{array}{c}
\mu_{I R F-M} \\
\mu_{I M A-B} \\
\mu_{D O L} \\
\mu_{I B O V}
\end{array}\right]+\left[\begin{array}{c}
\epsilon_{1} \\
\epsilon_{2}
\end{array}\right]
$$




$$
\hat{\boldsymbol{\mu}}_{B L, 1}=\left[\begin{array}{llll}
0.6 \% & 1.3 \% & 1.4 \% & 3.17 \%
\end{array}\right]^{\prime} .
$$

Suppose that the second analyst is more optimistic about the stock market and less optimistic about inflation-indexed bonds. Thus, he expresses his views mathematically in the Black-Litterman framework as

$$
\left[\begin{array}{l}
0.5 \% \\
-4 \%
\end{array}\right]=\left[\begin{array}{cccc}
0 & 1 & 0 & 0 \\
0 & 0 & 1 & -1
\end{array}\right]\left[\begin{array}{c}
\mu_{I R F-M} \\
\mu_{I M A-B} \\
\mu_{D O L} \\
\mu_{I B O V}
\end{array}\right]+\left[\begin{array}{l}
\epsilon_{1} \\
\epsilon_{2}
\end{array}\right]
$$

using these views the calculation of Black-Litterman excess returns leads to

$$
\hat{\boldsymbol{\mu}}_{B L, 2}=\left[\begin{array}{llll}
0.4 \% & 0.76 \% & -0.05 \% & 5.0 \%
\end{array}\right]^{\prime} .
$$

To demonstrate that the robust approach also leads to rather different portfolio allocations compared to the classical setting, we perform analogous calculations as in Example 1. In figure 3.1, we see this uncertainty set resulted in a significant change in the portfolio allocations in comparison to the classical one. In our example, the robust problem reduced the allocation in fixed income and inflation-index bonds, due to the pessimistic view of analyst 2 . Furthermore, the allocation in US Dollar had an expressive increase for high levels of volatility, attributed to its negative correlation with all other assets, which is consistent with diversification principles 

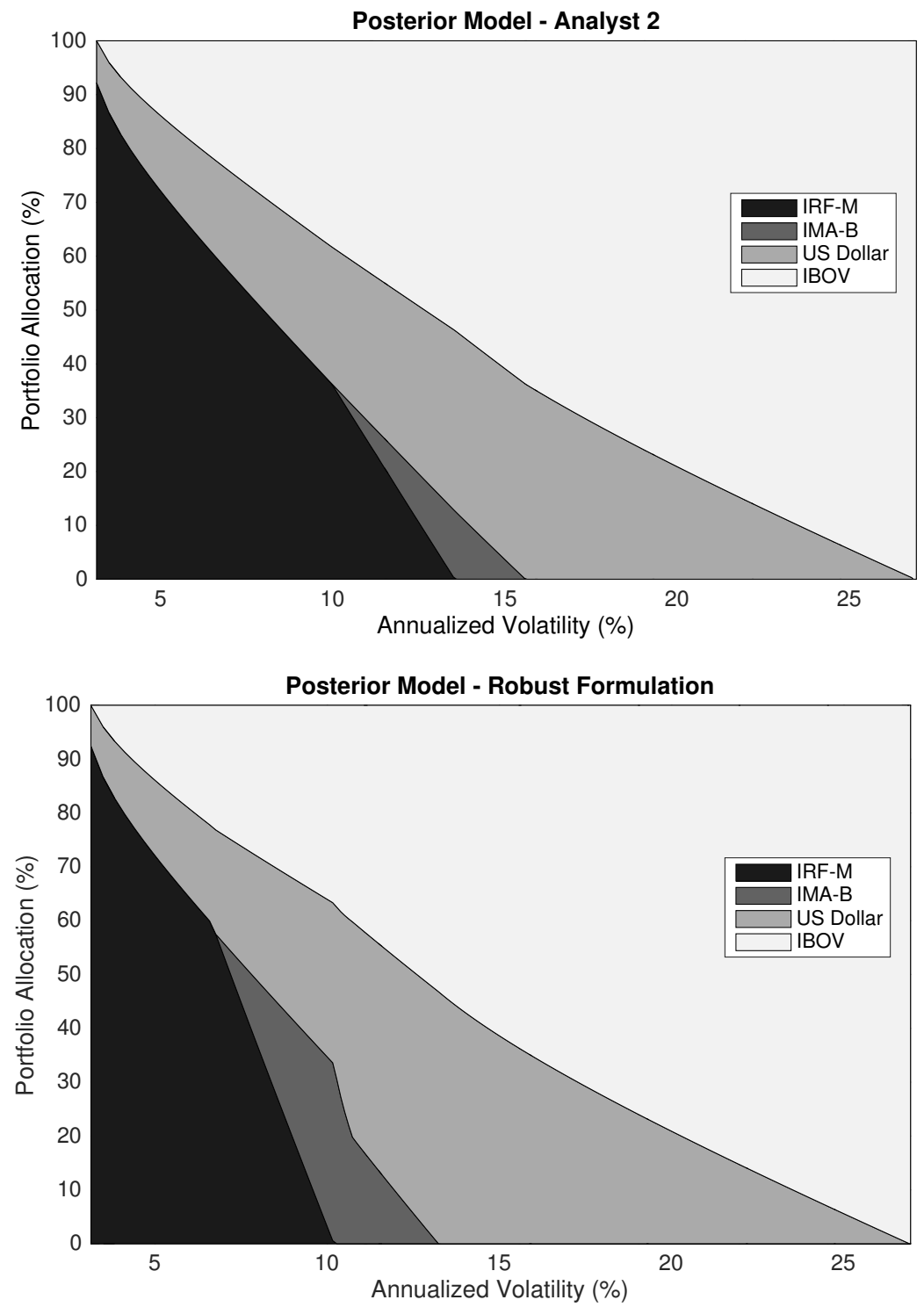

Figure 3.1: Classical and Robust Black-Litterman allocations

\section{3}

\section{Robust Black-Litterman with Incomplete Information}

In this section we start to explore models with incomplete information about the views. The ideas presented in this section can be used by investors to employ views from market polls, in the robust Black-Litterman model. We provide two methodologies to model uncertainty sets from a sample of market participants. 


\subsection{1}

\section{Bertsimas and Sim's Uncertainty Set}

Suppose now that there are incomplete information about the views and the investor only has the maximum, minimum and nominal values (i.e. average or median value) of the views on the future asset returns from the forecasters, for the same portfolio $\mathbf{P} \in \mathbb{R}^{m \times n}$ and covariance matrix $\Omega \in \mathbb{R}^{m \times m}$. In this scenario, our second model is based on the robust optimization framework proposed by Bertsimas and Sim (2004). Their approach retains the advantages of the linear formulation proposed by Soyster (1973), in addition offers a methodology to control the degree of robustness for every constraint by introducing the parameter $\Gamma$, that, in our model, take a real value on the interval $[0, m]$. The problem is formulated to protect deterministically against worst case violation of the $i$ constraint, only when a predetermined number of $\Gamma$ uncertainty coefficients are allowed to change. In other words, $\Gamma$ controls the number of uncertainty coefficients that may deviate from the nominal value.

Using the robust formulation proposed by Bertsimas and Sim (2004), the uncertainty set for a general correlated set of views can be modeled as

$$
\mathcal{Q}_{q}=\left\{\mathbf{q}: \mathbf{q}=\hat{\mathbf{q}}+\mathbf{C}_{q}^{-1 / 2} \boldsymbol{\eta} \mid \mathbf{z} \circ(\overline{\mathbf{q}}-\hat{\mathbf{q}}) \geq \boldsymbol{\eta} \geq \mathbf{z} \circ(\underline{\mathbf{q}}-\hat{\mathbf{q}}), \mathbf{z}^{\prime} \mathbf{1} \leq \Gamma, 0 \leq \mathbf{z} \leq 1\right\},
$$

where $\mathbf{C}_{q}^{-1 / 2}$ is the Cholesky decomposition of the views's correlation matrix $\mathbf{C}_{q}, \boldsymbol{\eta}$ is the parameter that controls the uncertainty on the views $\mathbf{q}, \Gamma$ is the parameter introduced by Bertsimas and Sim (2004) known as uncertainty budget, $\overline{\mathbf{q}}$ is the upper bound, $\underline{\mathbf{q}}$ is the lower bound, $\hat{\mathbf{q}}$ is the nominal value and $\circ$ is an element-wise product of two matrices (Hadamard Product).

In order to formulate problem (3-2) as a one-level optimization problem under this uncertainty set, consider the following linear optimization model

$$
\begin{array}{cl}
\underset{z, \eta}{\operatorname{minimize}} & \left(\mathbf{a}+\mathbf{B}\left(\hat{\mathbf{q}}+\mathbf{C}_{q}^{-1 / 2} \boldsymbol{\eta}\right)\right)^{\prime} \mathbf{x} \\
\text { subject to } & \eta_{i} \geq z_{i}\left(\underline{q}_{i}-\hat{q}_{i}\right), \forall i: \overline{\boldsymbol{\phi}} \\
& \eta_{i} \leq z_{i}\left(\bar{q}_{i}-\hat{q}_{i}\right), \forall i: \underline{\boldsymbol{\phi}} \\
& \sum_{i=1}^{m} z_{i} \leq \Gamma: \lambda \\
& \mathbf{z} \leq \mathbf{1}: \boldsymbol{\delta} \\
& \mathbf{z} \geq \mathbf{0},
\end{array}
$$

where $\overline{\boldsymbol{\phi}}, \underline{\boldsymbol{\phi}}, \lambda$ and $\boldsymbol{\delta}$ are the dual variables associated to each constraint of the problem. The dual problem of (3-14) is given by 


$$
\begin{array}{cl}
\underset{\lambda, \overline{\boldsymbol{\phi}}, \underline{\phi}, \delta}{\operatorname{maximize}} & \hat{\boldsymbol{\mu}}_{B L}^{\prime} \mathbf{x}-\Gamma \lambda-\mathbf{1}^{\prime} \boldsymbol{\delta} \\
\text { subject to } & \bar{\phi}_{i}\left(\bar{q}_{i}-\hat{q}_{i}\right)-\underline{\phi}_{i}\left(\underline{q}_{i}-\hat{q}_{i}\right)+\lambda+\delta_{i} \geq 0, \forall i \\
& \underline{\boldsymbol{\phi}}-\overline{\boldsymbol{\phi}}=\mathbf{C}_{q}^{1 / 2} \mathbf{B}^{\prime} \mathbf{x}, \\
& \overline{\boldsymbol{\phi}} \geq 0, \underline{\boldsymbol{\phi}} \geq 0, \lambda \geq 0, \boldsymbol{\delta} \geq 0
\end{array}
$$

Since problem (3-14) is convex, feasible and bounded for all $\Gamma \in[0, m]$, by strong duality problem (3-15) is also bounded and feasible and their optimal values are the same. Therefore, substituting problem (3-14) to its dual we arrive at the following equivalent one-level allocation problem

$$
\begin{array}{cl}
\underset{\lambda, \bar{\phi}, \underline{\phi}, \delta}{\operatorname{minimize}} & \mathbf{x}^{\prime} \boldsymbol{\Sigma}_{B L} \mathbf{x} \\
\text { subject to } & \hat{\boldsymbol{\mu}}_{B L}^{\prime} \mathbf{x}-\Gamma \lambda-\mathbf{1}^{\prime} \boldsymbol{\delta} \geq \mu_{0} \\
& \bar{\phi}_{i}\left(\bar{q}_{i}-\hat{q}_{i}\right)-\underline{\phi}_{i}\left(\underline{q}_{i}-\hat{q}_{i}\right)+\lambda+\delta_{i} \geq 0, \forall i \\
& \underline{\boldsymbol{\phi}}-\overline{\boldsymbol{\phi}}=\mathbf{C}_{q}^{1 / 2} \mathbf{B}^{\prime} \mathbf{x} \\
& \overline{\boldsymbol{\phi}} \geq 0, \underline{\boldsymbol{\phi}} \geq 0, \lambda \geq 0, \boldsymbol{\delta} \geq 0 .
\end{array}
$$

This general robust formulation allows the decision maker to input upper bounds and lower bounds on each view. In addition, using the budget of uncertainty $\Gamma$, it is also possible to control the number of views that might take their worst value simultaneously.

\subsection{2}

\section{Ellipsoidal Uncertainty Sets}

We motivate our next model as follows. Let's consider two possible scenarios, first the manager has only the average return view $\hat{\mathbf{q}} \in \mathbb{R}$ of $N$ different independent identically distributed portfolio views, on the same portfolio $\mathbf{P} \in \mathbb{R}^{m \times n}$ and known confidence covariance matrix $\Omega \in \mathbb{R}^{m \times m}$. In the second case, we also assume that the investor has information on the covariance matrix of the forecasts, which we denote $\mathbf{S}_{\mathbf{q}} \in \mathbb{R}^{m \times m}$. Note that the covariance matrix in average return forecasts is not necessarily the same as the confidence covariance matrix. Throughout this section we provide insights on how to use these informations on our general robust model (3-2).

We start with the first scenario. To use the information on average return of the views and the number of forecasters $N$ as a robust form of the BlackLitterman model, we provide a new perspective in light of hypothesis testing. For that, consider the hypotheses

$$
H_{o}: \boldsymbol{\mu}_{B L}=\hat{\boldsymbol{\mu}}_{B L}^{M L} \quad \text { vs } \quad H_{a}: \boldsymbol{\mu}_{B L} \neq \hat{\boldsymbol{\mu}}_{B L}^{M L},
$$

where $\hat{\boldsymbol{\mu}}_{B L}^{M L}$ is the maximum likelihood estimated Black-Litterman return con- 
sidering the average return of $N$ forecasters. We consider that the distribution of the maximum likelihood estimator $\hat{\boldsymbol{\mu}}_{B L}$, based on an i.i.d sample of $N$ investors is given by

$$
\hat{\boldsymbol{\mu}}_{B L}^{M L} \sim \mathcal{N}\left(\hat{\boldsymbol{\mu}}_{B L}, \frac{\hat{\boldsymbol{\Sigma}}_{B L}^{\mu}}{N}\right)
$$

where, $\Sigma_{B L}^{\mu}$ is the covariance matrix obtained from the Bayesian update estimator in equation (2-62).

To create an uncertainty set around the vector of the posterior mean returns $\boldsymbol{\mu}_{B L}$ or, in the case of the Black-Litterman model, around the estimate $\hat{\boldsymbol{\mu}}_{B L}$ since the true market parameter is unknown, we need the distribution of $\hat{\boldsymbol{\mu}}_{B L}$. In case of elliptical distribution, information about the first two moments is sufficient to determine an ellipsoidal confidence interval. Therefore, it is possible to create a confidence ellipsoid centered at the point estimate $\hat{\boldsymbol{\mu}}_{B L}$ and using to describe the shape $\hat{\boldsymbol{\Sigma}}_{B L}$, thus

$$
\begin{aligned}
\mathcal{U}_{\boldsymbol{\mu}_{B L}} & \left.=\left\{\boldsymbol{\mu}_{B L} \in \mathbb{R}^{n} \mid\left(\boldsymbol{\mu}_{B L}-\mathbb{E}\left[\hat{\boldsymbol{\mu}}_{B L}^{M L}\right]\right)^{\prime}\left(\mathbf{C o v}\left[\hat{\boldsymbol{\mu}}_{B L}^{M L}\right]\right)^{-1}\left(\boldsymbol{\mu}_{B L}-\mathbb{E}\left[\hat{\boldsymbol{\mu}}_{B L}^{M L}\right]\right)\right) \leq \delta^{2}\right\} \\
& =\left\{\boldsymbol{\mu}_{B L} \in \mathbb{R}^{n} \mid\left(\boldsymbol{\mu}_{B L}-\hat{\boldsymbol{\mu}}_{B L}\right)^{\prime}\left(\frac{\boldsymbol{\Sigma}_{B L}^{\mu}}{N}\right)^{-1}\left(\boldsymbol{\mu}_{B L}-\hat{\boldsymbol{\mu}}_{B L}\right) \leq \delta^{2}\right\} \\
& =\left\{\boldsymbol{\mu}_{B L} \in \mathbb{R}^{n} \mid\left(\boldsymbol{\mu}_{B L}-\hat{\boldsymbol{\mu}}_{B L}\right)^{\prime}\left(\boldsymbol{\Sigma}_{B L}^{\boldsymbol{\mu}}\right)^{-1}\left(\boldsymbol{\mu}_{B L}-\hat{\boldsymbol{\mu}}_{B L}\right) \leq \delta^{2} / N\right\},
\end{aligned}
$$

where the size of $\delta^{2}$ determines the size of the uncertainty set and defines the desired confidence from the investor. In case of a multivariate normal distributions, $\left(\boldsymbol{\mu}_{B L}-\hat{\boldsymbol{\mu}}_{B L}\right)^{\prime}\left(\boldsymbol{\Sigma}_{B L}^{\mu}\right)^{-1}\left(\boldsymbol{\mu}_{B L}-\hat{\boldsymbol{\mu}}_{B L}\right)$ follows a chi-squared distribution with $n$ degrees of freedom. Thus, the size of $\delta^{2}$ can be defined appropriate by a confidence level $\alpha \in(0,1)$, such that $\delta^{2}=\chi_{n}^{2}(\alpha)$. In figure 3.2 we illustrate a multivariate normal setting in two dimensions originating ellipsoidal uncertainty sets for different values of $\alpha$. 

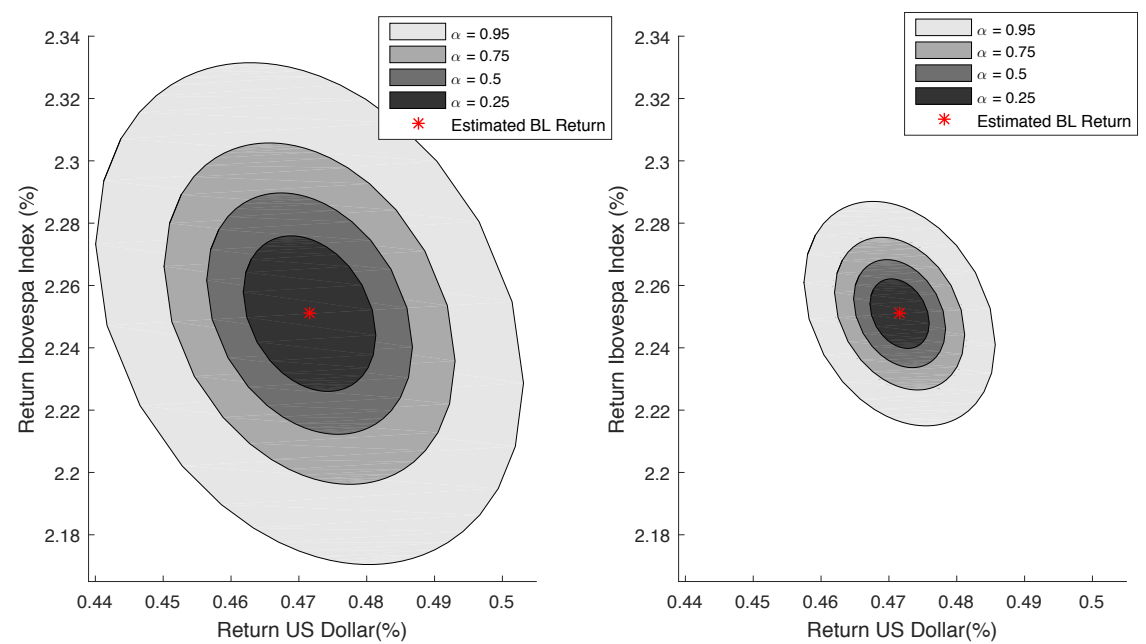

Figure 3.2: Example of Black-Litterman model using confidence ellipsoid. On the left: $\mathrm{N}=2$, on the right: $\mathrm{N}=10$.

Observe that from this figure we could also infer that the US Dollar has a smaller estimated volatility compared to Ibovespa index, as its respective axis of the ellipse is quite shorter. Moreover, the ellipse coordinate axes are tilted downward showing that the two assets are negatively correlated. And, more importantly, we notice that the uncertainty set reduces as the number of forecasters $\mathrm{N}$ increases, resembling a higher confidence for a larger sample. Therefore, this setting is more appropriate when the decision maker is able to infer more accurate results for larger samples of forecasters without any extra information about the forecasts besides average return.

As was shown for problem (2-44), using an ellipsoidal uncertainty set the original mean variance problem (3-2) can be reduced to the following secondorder cone programming problem

$$
\begin{array}{ll}
\underset{\mathbf{x}}{\operatorname{minimize}} & \mathbf{x}^{\prime} \boldsymbol{\Sigma}_{B L} \mathbf{x} \\
\text { subject to } & \hat{\boldsymbol{\mu}}_{B L}^{\prime} \mathbf{x}-\frac{\chi_{n}^{2}(\alpha)}{N}\left\|\left(\boldsymbol{\Sigma}_{B L}^{\mu}\right)^{1 / 2} \mathbf{x}\right\|_{2} \geq \mu_{0} .
\end{array}
$$

A quite interesting result can be found in this particular setting: the gap between the robust efficient frontier and the classical Black-Litterman frontier increases with respect to the risk axis, to, the optimal porfolio tends to be relatively more conservative for higher levels of volatility. Also, for the same level of risk the investor always chooses a more conservative portfolio when performing a robust optimization. These results are illustrated in figure 3.3 using the same two assets from the previous example, confidence level $\alpha=0.90$ and neither short selling or leveraging allowed. 


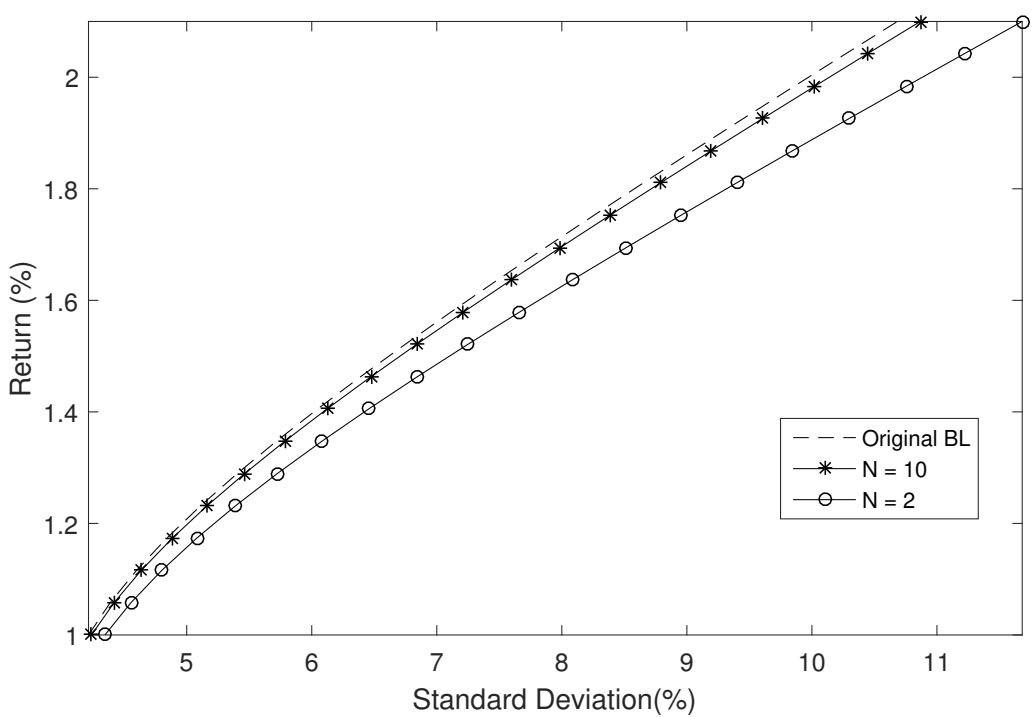

Figure 3.3: Efficient frontier for $\alpha=0.95$.

Besides this general ellipsoidal $\boldsymbol{\mu}_{B L}$ uncertainty set, one could use the robust optimization approach to model only the uncertainty related to the views $\mathbf{q} \in \mathbb{R}^{m}$, therefore not considering the variance of the mean return estimator. A similar approach to the previous model can be performed. We assume that the views forecast would describe an ellipsoidal uncertainty with the same shape of the confidence covariance matrix $\Omega$, is this case the uncertainty set would be:

$$
\mathcal{Q}_{q}=\left\{\mathbf{q} \in \mathbb{R}^{m} \mid(\mathbf{q}-\hat{\mathbf{q}})^{\prime} \mathbf{\Omega}^{-1}(\mathbf{q}-\hat{\mathbf{q}}) \leq \frac{\chi_{m}^{2}(\alpha)}{N}\right\}
$$

Using this uncertainty we can represent the inner problem of (3-2) as

$$
\begin{array}{ll}
\underset{\mathbf{q}}{\operatorname{minimize}} & (\mathbf{a}+\mathbf{B q})^{\prime} x \\
\text { subject to } & \left\|\boldsymbol{\Omega}^{-1 / 2}(\mathbf{q}-\hat{\mathbf{q}})\right\|_{2}^{2} \leq \frac{\chi_{n}^{2}(\alpha)}{N},
\end{array}
$$

where $\Omega^{-1 / 2} \in \mathbb{R}^{m \times m}$ is the lower triangular matrix from the Cholesky decomposition of $\Omega^{-1}$. Following the methodology presented in section 2.1.2, we find that the closed form solution of the SOCP dual problem is

$$
\hat{\boldsymbol{\mu}}_{B L}^{\prime} \mathbf{x}-\frac{\chi_{m}^{2}(\alpha)}{N}\left\|\boldsymbol{\Omega}^{1 / 2} \mathbf{B}^{\prime} \mathbf{x}\right\|_{2}
$$

hence, the robust problem formulation of (3-2) becomes

$$
\begin{array}{ll}
\underset{\mathbf{x}}{\operatorname{minimize}} & \mathbf{x}^{\prime} \boldsymbol{\Sigma}_{B L} \mathbf{x} \\
\text { subject to } & \hat{\boldsymbol{\mu}}_{B L}^{\prime} \mathbf{x}-\frac{\chi_{m}^{2}(\alpha)}{N}\left\|\boldsymbol{\Omega}^{1 / 2} \mathbf{B}^{\prime} \mathbf{x}\right\|_{2} \geq \mu_{0} .
\end{array}
$$


In these two robust models we see a very intuitive interpretation of the uncertainty set. As the number of forecasters $N$ increases, the uncertainty set reduces and the robust model converges to the original Black-Litterman model. However for a small number of forecasters $N$, the uncertainty set becomes larger enough to account for possible estimation errors.

In the second case we assume now that we actually have the information about the covariance matrix of the forecasts. An investor with multiple forecasts information may be uncomfortable specifying the average view $\hat{\mathbf{q}}$. Rather, one might specify an uncertainty set that captures the actual dispersion of the views and constrain the portfolio to incur in all the possible scenarios set by the analysts with a particular level of confidence. In this case, the investor might form a portfolio robust to uncertainty market views.

Suppose that the decision maker believes that the conflicting forecasts are distributed as a multivariate normal distribution with covariance matrix $\mathbf{S}_{\mathbf{q}}$ and average $\hat{\mathbf{q}}$. Thus, it is natural to assume an ellipsoidal uncertainty set for the views

$$
\mathcal{Q}_{q}=\left\{\mathbf{q} \in \mathbb{R}^{m} \mid(\mathbf{q}-\hat{\mathbf{q}})^{\prime} \mathbf{S}_{\mathbf{q}}^{-1}(\mathbf{q}-\hat{\mathbf{q}}) \leq \chi_{m}^{2}(\alpha)\right\} .
$$

With this uncertainty set problem (3-2) reduces to the following

$$
\begin{array}{ll}
\underset{\mathbf{x}}{\operatorname{minimize}} & \mathbf{x}^{\prime} \boldsymbol{\Sigma}_{B L} \mathbf{x} \\
\text { subject to } & \hat{\boldsymbol{\mu}}_{B L}^{\prime} \mathbf{x}-\chi_{m}^{2}(\alpha)\left\|\mathbf{S}_{\mathbf{q}}^{1 / 2} \mathbf{B}^{\prime} \mathbf{x}\right\|_{2} \geq \mu_{0} .
\end{array}
$$

Analogous to the models presented in the previous sections, this model allow to define an uncertainty structure of $\mathbf{q}$ that is independent of the inputs defined in the Black-Litterman model, leading to optimal solutions. In the next chapters we empirically study each robust model that we have proposed and present situations where each model might be useful. 


\section{4}

\section{Controlled Tests}

In this section we empirically study how the models behave with a small number of forecasters (i.e. 5) as the uncertainty of their views increased by the parameter, which here we denominate as $\tau_{\mathbf{q}}$. We also investigate how the precision on the views influences each robust model and the original BlackLitterman. The robust approach generalizes the traditional Black-Litterman methodology, where the uncertainty sets are defined as a single point estimate. However, the role of these numerical tests is not to evaluate which is the best model, but rather to help understand when and how each model can be used as a better alternative.

For simplicity, we consider the scenario where the returns follow a multivariate normal distribution. Furthermore, we assume that the CAPM equilibrium model is estimated by a simulated returns from this distribution. Then, we test the proposed robust models and the original using synthetic data and manipulated examples. Using this setting, our controlled numerical tests are divided in two experiments. First, we compare the performance of the models when views are static and in average correct to a similar case where the views are static and in average incorrect, for various levels of uncertainty in the views. This example aims to simulate different scenarios of specialist views (i.e. correct and incorrect in average) and see how it effects each model.

In our second test we want to measure the actual impact of views's accuracy on the expected returns. In this case we consider fixed levels for the uncertainty parameter $\tau_{\mathbf{q}}$ and stress the accuracy of the forecasts. In this experiment, we also compare the out of sample performance of the portfolios using all robust methods. In the next section, we present in detail the assumptions used in each experiment.

\section{1}

\section{Experiment Setup}

We illustrate both examples using a model with 4 risky assets and a riskfree asset. To simplify, the rate of return of the risk-free asset is fixed at zero. We consider that the investor is not allowed to short sell positions, thus the wealth allocation has the following setting

$$
\mathcal{X}=\left\{\mathrm{x} \in \mathbb{R}^{4} \mid \mathrm{x} \geq 0, \mathbf{1}^{\prime} \mathbf{x}=1\right\}
$$


The returns of the four risky assets are assumed as multivariate normal. In percentages, the nominal returns $(\boldsymbol{\mu})$ and variances $(\boldsymbol{\sigma})$ of each asset are taken as

$$
\begin{aligned}
\boldsymbol{\mu} & =\left[\begin{array}{llll}
0.85 & 0.89 & 3.87 & 0.40
\end{array}\right]^{\prime}, \\
\boldsymbol{\sigma} & =\left[\begin{array}{llll}
8.7 & 18.4 & 26.56 & 9.56
\end{array}\right]^{\prime},
\end{aligned}
$$

and, correlation matrix chosen as

$$
\boldsymbol{C}=\left[\begin{array}{cccc}
1 & -0.25 & 0.45 & -0.15 \\
& 1 & -0.35 & 0.18 \\
& & 1 & -0.15 \\
& & & 1
\end{array}\right]
$$

The assumption of a normal market implies that the mean-variance framework is the optimal allocation for any set of investor preferences. For instance, consider a portfolio with target variance $\sigma_{\text {target }}=8 \%$ per annum, then, the theoretical optimal portfolio would be $x_{\text {opt }}=(0.27,0.29,0.43,0)$. We are interested to see how each model performs under different scenario.

The experiments will go as follows:

- Market and Black-Litterman Assumptions

1. We simulate a sample of $N=60$ observations from the multivariate distribution with mean and covariance matrix as defined;

2. We maximize the return in the Robust mean-variance BlackLitterman model for standard deviation target of $8 \%$ (i.e. $\sigma \leq 8 \%$ ). Without loss of generality we solve the following reformulation of general robust problem 3-1

$$
\begin{array}{ll}
\underset{\mathbf{x}}{\operatorname{maximize}} & t \\
\text { subject to } & \boldsymbol{\mu}_{B L}(\mathbf{q})^{\prime} \mathbf{x} \geq t, \forall \mathbf{q} \in \mathcal{Q}_{q} \\
& \mathbf{x}^{\prime} \hat{\boldsymbol{\Sigma}}_{B L} \mathbf{x} \leq(8 \%)^{2} \\
& \mathbf{x}^{\prime} \mathbf{1}=1 \\
& \mathbf{x} \geq 0
\end{array}
$$

3. We set the CAPM as the sample mean and sample covariance matrix from the simulated data, thus

$$
\begin{gathered}
\boldsymbol{\pi}=\frac{1}{N} \sum_{i=1}^{N} \boldsymbol{\mu}_{i}, \\
\hat{\boldsymbol{\Sigma}}=\frac{1}{N-1} \sum_{i=1}^{N}\left(\boldsymbol{\mu}_{i}-\overline{\boldsymbol{\mu}}\right)\left(\boldsymbol{\mu}_{i}-\overline{\boldsymbol{\mu}}\right)^{\prime},
\end{gathered}
$$


and the Black-Litterman parameter $\tau=0.05$ as in He and Litterman (1999).

4. We use the same market assumption and optimization model in both experiments

- Views and Forecasters Assumptions

1. We assume a scenario of a hedge fund with 5 analysts, where each of them has two views on the following portfolio $\mathbf{P}$ :

$$
\mathbf{P}=\left[\begin{array}{cccc}
1 & 1 & 0 & -1 \\
0 & -1 & 1 & 0
\end{array}\right]
$$

2. The views on the aforementioned portfolio are randomly simulated as the following distribution:

$$
\mathbf{q}_{i}=\hat{\mathbf{q}}+\boldsymbol{e}_{q} \sim N\left(0, \tau_{\mathbf{q}} \mathbf{P} \Sigma \mathbf{P}^{\prime}\right),
$$

where, $\tau_{\mathbf{q}}$ is the parameter is added to the experiment to control the dispersion of the views, notice that it is independent of $\tau$ which is a parameter that determines the uncertainty of the average market equilibrium returns. Therefore, for larger values of $\tau_{\mathbf{q}}$ the uncertainty sets on the robust models increases correspondingly. Moreover, the average view controls the accuracy of the forecasts, for example, when $\hat{\mathbf{q}}=\mathbf{P} \boldsymbol{\mu}$ the forecasters are generally correct about the returns. The covariance matrix of the forecasters's views are not completely random, we assume that it follows the market dynamics scaled by the uncertainty parameters $\tau_{\mathbf{q}}$.

3. The confidence matrix for each generated view is defined as $\Omega=$ $\mathbf{P}(\tau \hat{\boldsymbol{\Sigma}}) \mathbf{P}^{\prime} / 10$, implying a belief ten times stronger than the CAPM estimate with the same market dynamics.

- Views on Experiment 1 - Sensitivity to Uncertainty of the Views

1. In this experiment we consider two realizations for the average forecast on the portfolio. In the first scenario, we assume in average a perfect foresight, therefore, generating unbiased expected returns in the posterior distribution. For simulations purposes, we consider that each forecast $\mathbf{q}_{i}$ follows a multivariate normal distribution: 


$$
\begin{aligned}
\mathbf{q}_{i} & =\mathbf{P} \boldsymbol{\mu}+\boldsymbol{e}_{q} \sim N\left(0, \tau_{\mathbf{q}} \mathbf{P} \boldsymbol{\Sigma} \mathbf{P}^{\prime}\right) \\
& =\left[\begin{array}{l}
2.1 \\
3.0
\end{array}\right]+\boldsymbol{e}_{q} \sim N\left(0, \tau_{\mathbf{q}} \mathbf{P} \boldsymbol{\Sigma} \mathbf{P}^{\prime}\right)
\end{aligned}
$$

2. The second setting assumes that analysts are systematically wrong about their views, which we express as the following

$$
\mathbf{q}_{i}=\left[\begin{array}{l}
0 \\
0
\end{array}\right]+\boldsymbol{e}_{q} \sim N\left(0, \tau_{\mathbf{q}} \mathbf{P} \boldsymbol{\Sigma} \mathbf{P}^{\prime}\right) .
$$

The parameter $\tau_{\mathbf{q}}$ defines the size of the uncertainty sets. Our objective here is to test the uncertainty sets under different values of $\tau_{\mathbf{q}}$ and from this experiment to have a better understanding of their benefits. To illustrate, in figure 4.1 we display the $99 \%$ confidence interval of $\mathbf{q}$ for $\tau_{\mathbf{q}}=\{0.1,0.25\}$.

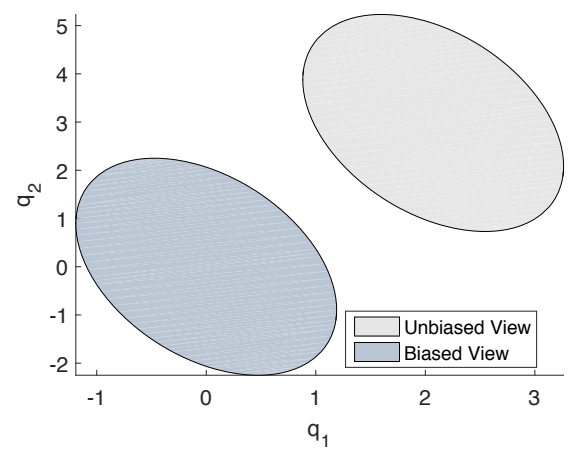

a) $\tau_{\mathbf{q}}=0.1$

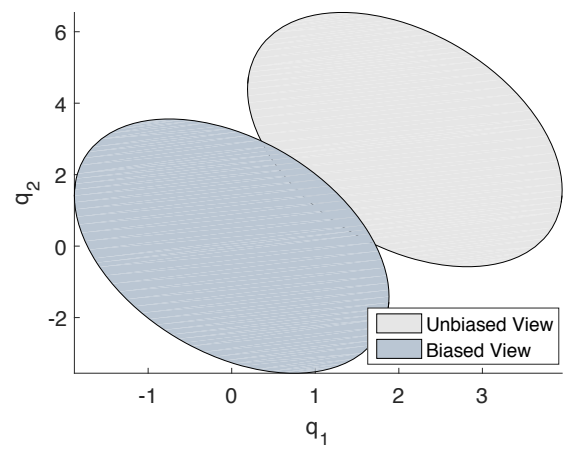

b) $\tau_{\mathbf{q}}=0.25$

Figure 4.1: Confidence interval for $\tau_{\mathbf{q}}$.

3. We simulate this experiment for $\tau_{\mathbf{q}}$ varying from 0.005 to 5 .

- Views on Experiment 2 - Sensitivity to Accuracy of the Forecasters

1. In the second experiment we consider multiples scenarios for $\hat{\mathbf{q}}$ as a linear function of the optimal value $\mathbf{P} \boldsymbol{\mu}$. Thus, the views on the portfolio $\mathbf{P}$ are simulated as

$$
\mathbf{q}_{i}=\hat{\mathbf{q}}\left(\xi_{\mathbf{q}}\right)+\boldsymbol{e}_{q} \sim N\left(0, \tau_{\mathbf{q}} \mathbf{P} \boldsymbol{\Sigma} \mathbf{P}^{\prime}\right)
$$

where $\hat{\mathbf{q}}\left(\xi_{\mathbf{q}}\right)=\xi_{\mathbf{q}}$ and $\xi_{\mathbf{q}}$ is used to control the accuracy of the forecasters.

2. We perform this experiment varying the accuracy parameter $\xi_{\mathbf{q}}$ linearly from -2 to 2 . 
3. We repeat this experiment for the following values of the uncertainty parameter: $\tau_{\mathbf{q}}=(0.5,1,2,4)$.

- Simulations and Statistics

1. We simulate the market returns and forecasts 500 times, for each simulation we calculate the out of sample return and standard deviation from the optimal strategy;

2. We compute the following statistics from the data set of portfolio returns:

- Mean: average observed out of sample portfolio return for 500 simulations;

- Standard deviation: standard deviation of the portfolio return over all simulations;

- Minimum return and maximum return portfolio returns observed over all simulations;

- Sharpe Ratio: ratio between mean and standard deviation;

- Empirical constraint-violation probability: empirical probability that the portfolio out of sample standard deviation goes above $8 \%$. It is obtained by the ratio between the number of observations that violated the standard deviation constraint (i.e. $\sigma>8 \%$ ) and the number of simulations (i.e. 500).

- Assumptions on Uncertainty sets

1. Multiple Forecasters: we assume perfect information on all five views from the simulated forecasters;

2. Bertsimas and Sims uncertainty set: in this case, the investor only has access to the average, maximum and minimum value of each view;

3. Ellipsoidal uncertainty set: we use the three proposed models. Therefore, we assume that the there is only information about the number of investors and their average view on the simulations for models 3-18 and 3-21 and for model 3-22 we also assume that the investor has information about the covariance matrix of the forecasters. For all models we assume a confidence level $\alpha$ of 0.95 ;

4. We compare the uncertainty sets under this assumptions against the original Black-Litterman using the average of all 5 views as an input. 


\section{2}

\section{Sensitivity to Uncertainty of the Views}

In this section we analyze the results of robust and traditional BlackLitterman methodologies, when applying the empirical tests from a multivariate Normal distribution. All the numerical results are reported in tables A.1 - A.7, from appendix A. Here, we refer to experiments where the forecasters are consistently wrong about the views with the label "With bias", and experiments when on average the forecasters are right about the views as "Without Bias". We also distinguish the ellipsoidal uncertainty sets thoughtout the figures of this section, we use Ellipsoidal $\left(\mu_{B L}\right)$, Ellipsoidal $(q)$ and $\operatorname{Ellipsoidal}\left(S_{q}\right)$ to refer models 3-18, 3-21 and 3-22 from section 3.3.2.

In figure 4.2 we see that with and without bias the average return decreases as the uncertainty parameter $\tau_{\mathbf{q}}$ grows. This result is expected. Intuitively as the views get more disperse, we observe a negative impact on the average out of sample results. However, the impacts change depending on the robustness of the model and on the precision of the views.

We observe that the average returns of the simulations in the models with multiple forecasters, Bertsimas uncertainty and Ellipsoidal $\left(S_{q}\right)$ sets are more sensitive to the uncertainty parameter $\tau_{\mathbf{q}}$. This is because the uncertainty sets on these models naturally grow with the dispersion of the views, thus, making more conservative allocations for a given level of volatility. And, in both cases the most conservative model (i.e. Bertsimas $\Gamma=2$ ) presented the lowest average return. We also observe the impact of the bias on the average return. With bias on the forecast the models have a high impact in low values of $\tau_{\mathbf{q}}$ and get more stable as the parameter increases, and Without bias the average return decreases almost monotonically for all models.

On the other hand, the uncertainty set of the ellipsoidal sets $\mu_{B L}, q$ and the original Black-Litterman remains the same for all $\tau_{\mathbf{q}}$. In this case, the worst impact is seen when the forecasts are done with bias. Whereas, when the views are in average right we note that the average returns are almost stable, only decreasing by a low rate. The stability of the average return also contrasts in both tests. In addition, for the value of the parameter $\tau$ and confidence of the views that was assumed, the results suggested that these models are more vulnerable to the impact of estimation errors of views. These first results are intended to show the trade-off between performance and robustness. It becomes clear that the robust models are more conservative, in case of where the return is maximized and the risk is constrained. 


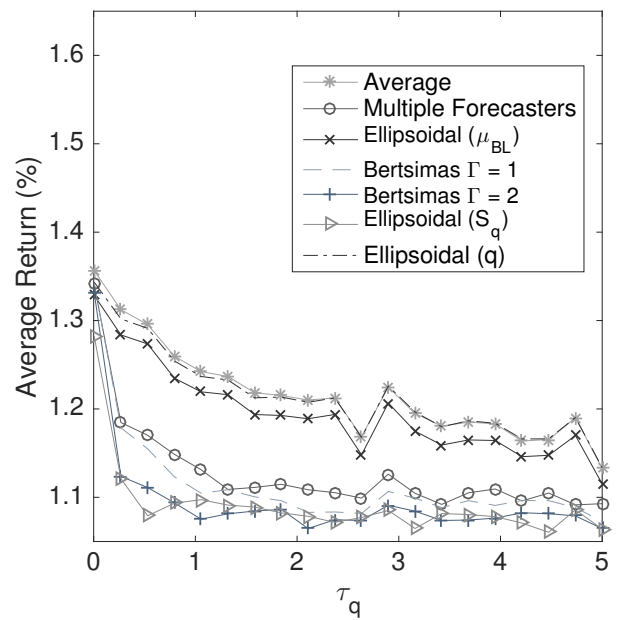

a) With bias

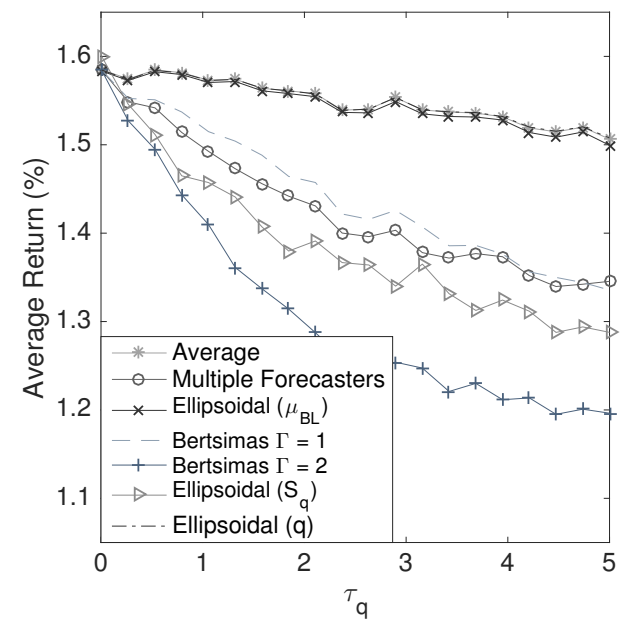

b) Without bias

Figure 4.2: Average return $\times \tau_{\mathbf{q}}$

The empirical probability gives a measure of how the feasibility of the variance constraint behaves under different possible scenarios. In figure 4.3, we observe that the empirical probability decreases for the models with multiple forecasters and Bertsimas and Sim's uncertainty sets and it drifts upwards for the other models. As the uncertainty set expands with $\tau_{\mathbf{q}}$, the robust models with multiple forecasters and Bertsimas uncertainty set reduces the risk of the allocations accordingly which increases the chances of an out sample variance below $8 \%$. Comparing with bias and without bias in these three robust models, we see a similar graphical pattern to the one observed in figure 4.2. This empirically shows that there is trade-off between average return and the price of robustness, which is independent of the bias. Moreover, we note that the price of robustness observed in the average return of the simulations might come at low cost for investors with tight volatility constraints. 


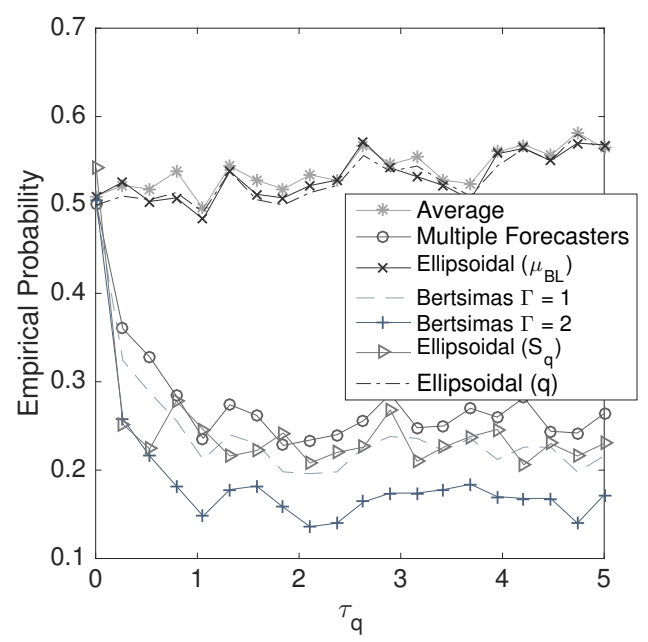

a) With bias

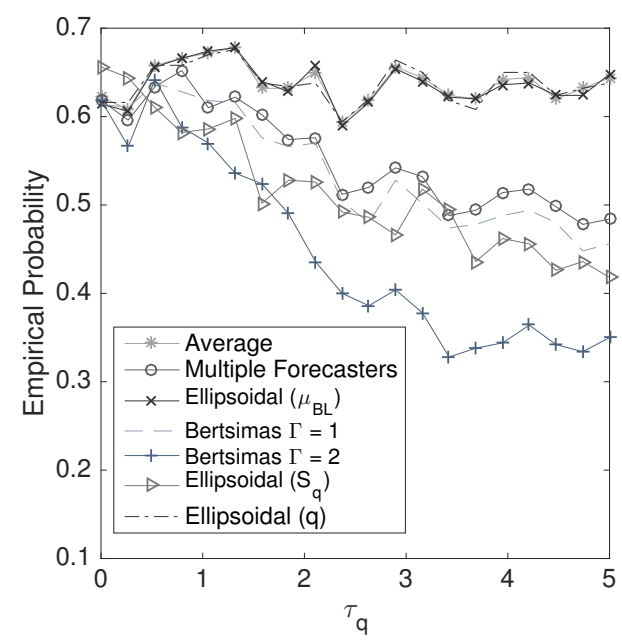

b) Without bias

Figure 4.3: Empirical probability $\times \tau_{\mathbf{q}}$

In order to obtain a higher average return, the original Black-Litterman and the robust models with ellipsoidal uncertainty sets $\mathbf{q}$ and $\mu_{B L}$ take more risk. This is corroborated by the higher levels of out of sample variance and empirical probabilities that can be seen with and without bias. We observe the extra risk is taken without taking into account the increasing uncertainty on the views. As a matter of fact, comparing the behavior of figure $4.3 \mathrm{a}$ ) and figure $4.2 a$ ), we see that the average return is highly penalized, whereas, the probability of constraint violation slightly increases. These results suggest that the approaches that considers more information about the forecasters are more robust to inaccuracy regarding the views.

Visual illustration of the results are presented in figures 4.4 and 4.5. We have taken $\tau_{\mathbf{q}}=5$. In the robust models with Multiple forecasts and Bertsimas uncertainty sets, we observe a concentration of the sample results around the $8 \%$ volatility level. These results are more evident when the forecasters are biased and on more conservative uncertainty sets (e.g. Bertsimas uncertainty sets in figure 4.4). 

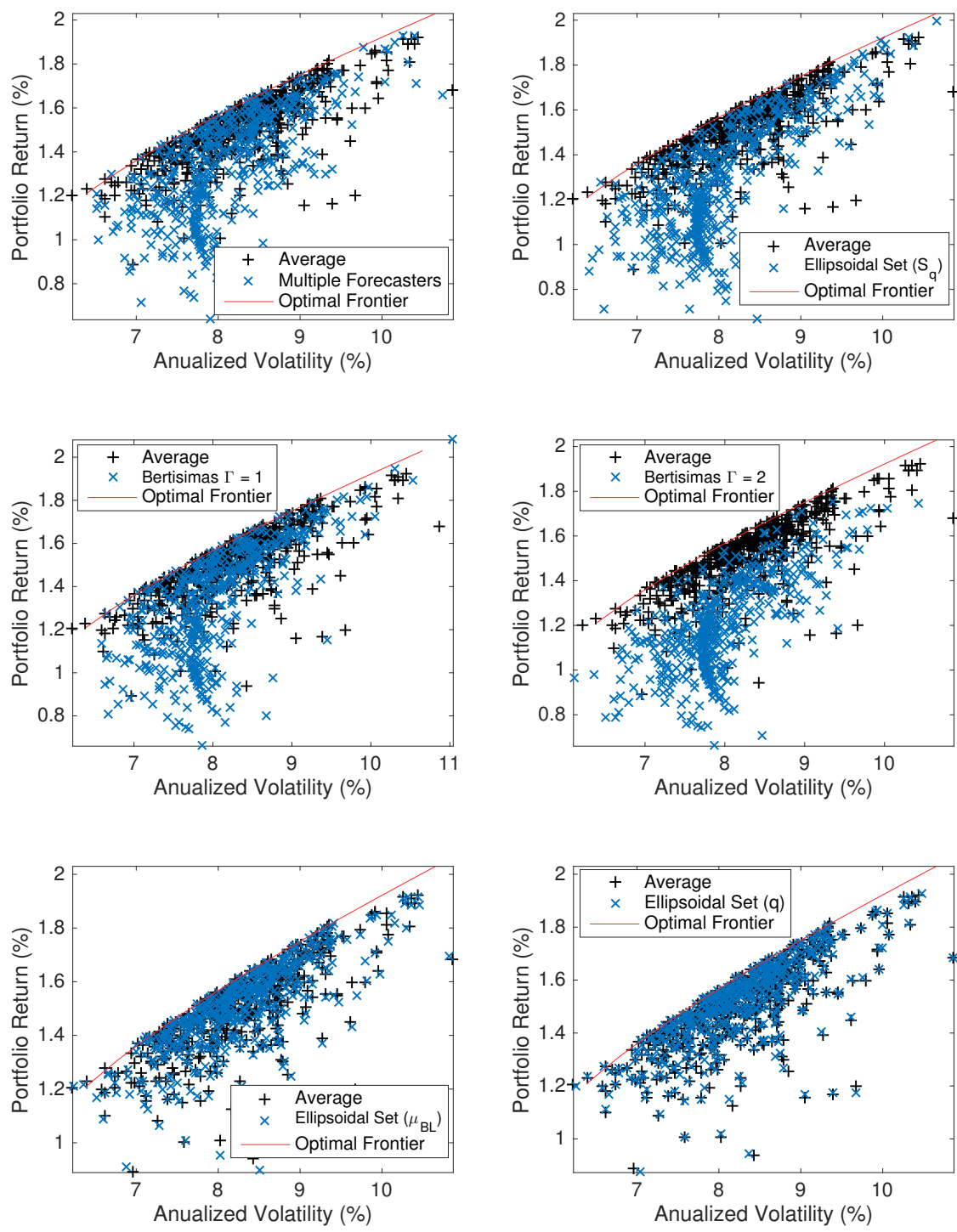

Figure 4.4: Out of sample standard deviation and portfolio returns without bias views for $\tau_{\mathbf{q}}=5$.

The models with ellipsoidal uncertainty sets $\left(\mu_{B L}\right.$ and $\left.q\right)$ display a more scattered behavior, similar to the single point average Black-Litterman in figure 4.5. In particular, these results occurred because these robust feasible sets are a function of the confidence covariance matrix $\Omega$ and the parameter $\tau$, which remained the same as the accuracy on the views gets worse. Therefore, the impact of a large uncertainty parameter $\tau_{\mathbf{q}}$ is not incorporated in the uncertainty sets. Consequently, the empirical probability was higher than 
the ones observed on other robust models, as it was previously mentioned. Furthermore, we see an agglomeration of points around the optimal frontier in figure 4.4. This is consistent with our intuition, when the investor is on average correct about his views we would expect a good overall performance. In figures 4.4 and 4.5 , we can visualize these insights.
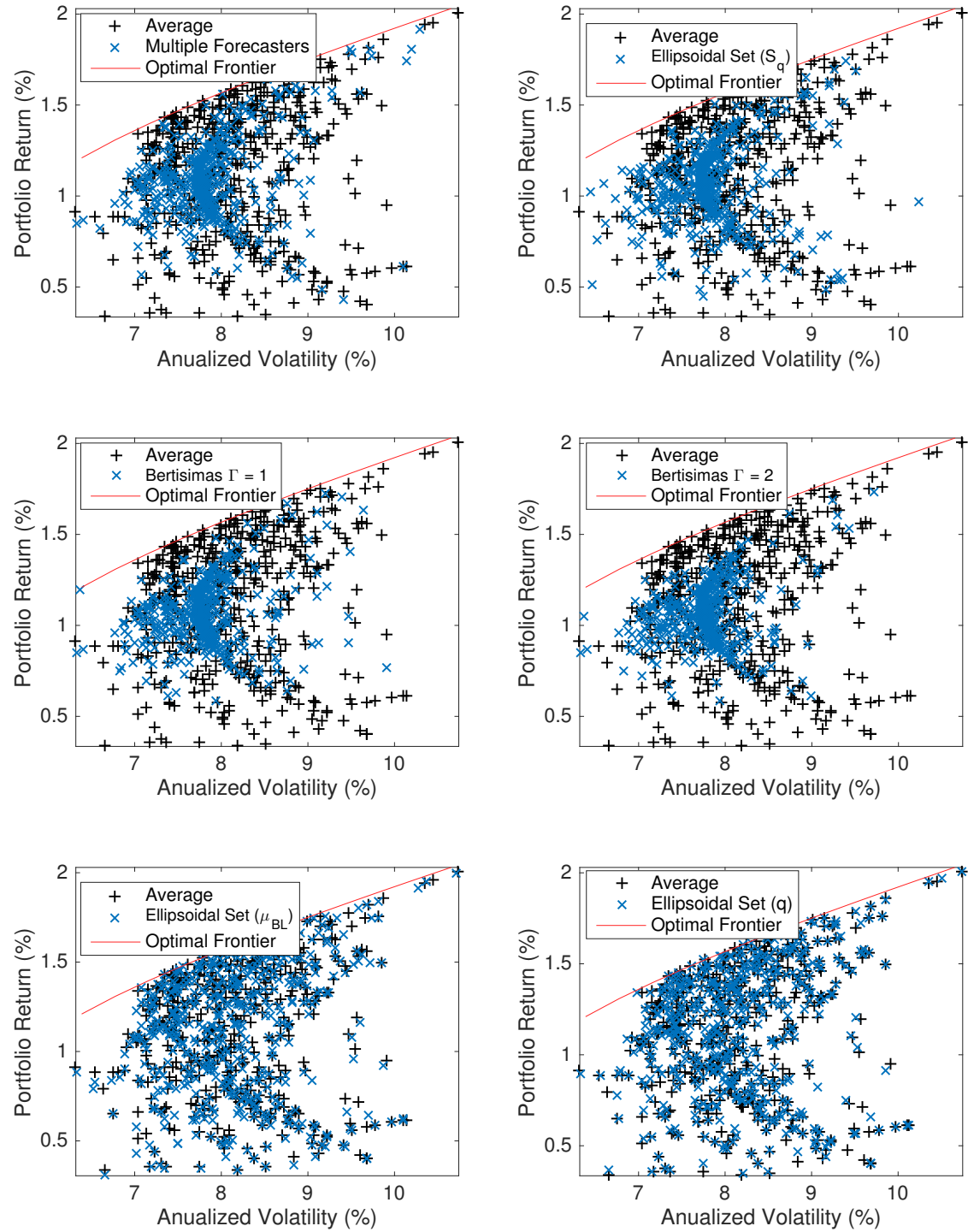

Figure 4.5: Out of sample standard deviation and portfolio returns with bias views for $\tau_{\mathbf{q}}=5$.

Figure 4.6 illustrates the evolution of the Sharpe ratio, which measures 
the efficiency of the robust models on each assumption. We observe there are opposite behaviors of the robust Black-Litterman methods as the uncertainty parameter increases. There are substantial Sharpe ratio loss associated with both the precision of the views and ignoring its uncertainty structure. Robust models takes into account $\tau_{q}$ has a more stable behavior when the views are biased, we even observe that for large values of $\tau_{q}$ the Sharpe ratio with or without bias converges to similar values. However, without bias we note that these models suffer a stronger impact, which is mostly due to a better overall result of the ellipsoidal uncertainty sets $q, \mu_{B L}$ and average Black-Litterman model under this assumption.

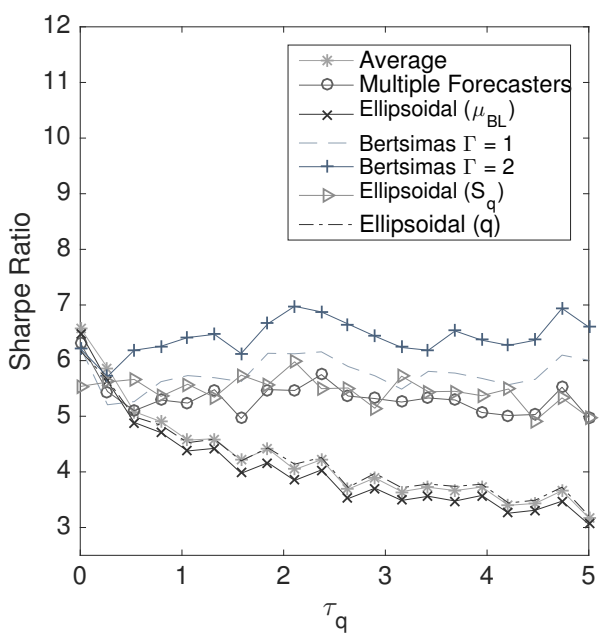

a) With bias

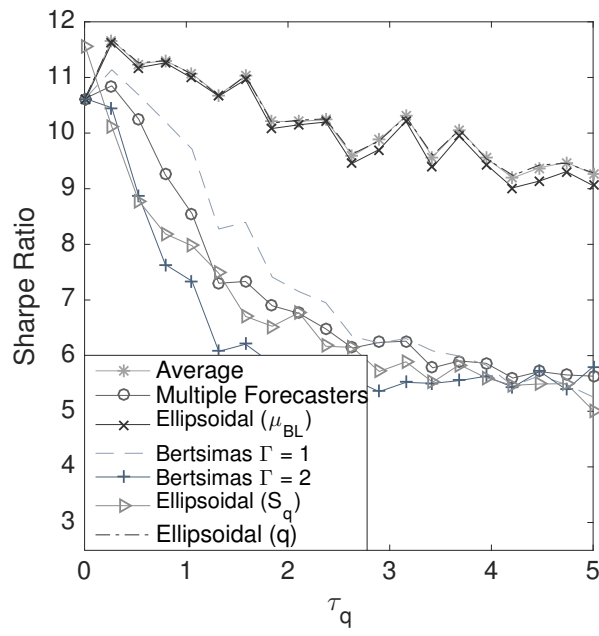

b) Without bias

Figure 4.6: Sharpe Ratio $\times \tau_{\mathbf{q}}$

The results for standard deviation and risk are quite surprising. In figure 4.7 , we see that as $\tau_{\mathbf{q}}$ increases the standard deviation of models Multiple Forecasters, Bertsimas and Ellipsoidal $\left(S_{q}\right)$ converges to almost the same values with and without bias. The robustness becomes more apparent as the uncertainty in that view increases. In special, Bertsimas $\Gamma=2$ has the lowest standard deviation when the views are biased. It is interesting to note that the largest uncertainty set becomes more conservative as uncertainty grows protecting against worst-case realizations of asset's returns.

As $\tau_{\mathbf{q}}$ increases, all three models that do not consider the uncertainty in the views have a lower standard deviation for unbiased forecasters. Note, however, in the biased scenario the standard deviation is higher compared to the robust models and never stabilizes, it keeps increasing as $\tau_{\mathbf{q}}$ goes to 5 . These results suggest that the robust approaches are appropriate to deal with inaccuracy and uncertainty in the views. 


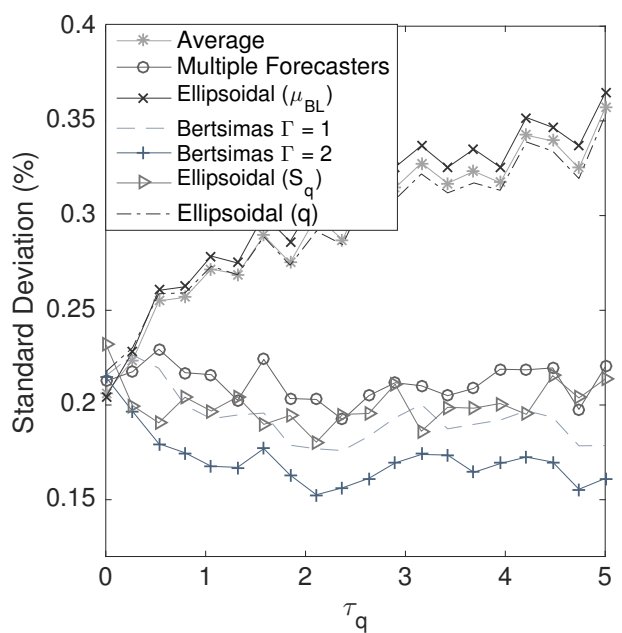

a) With bias

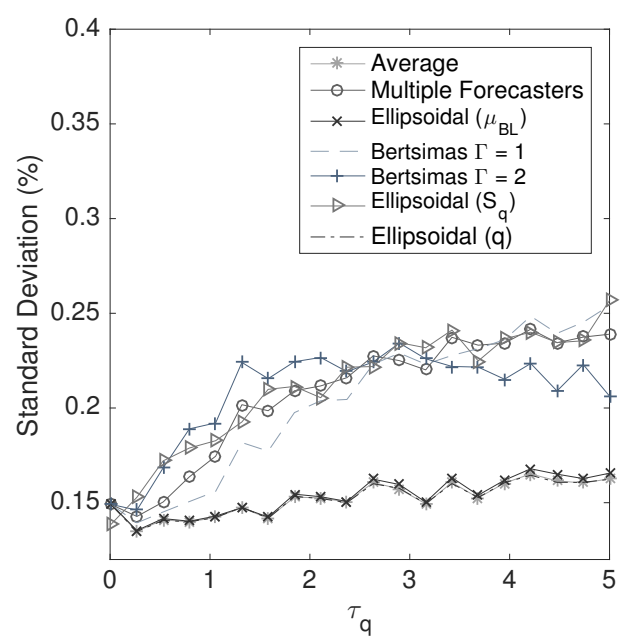

b) Without bias

Figure 4.7: Standard deviation of the simulations $\times \tau_{\mathbf{q}}$

Summarizing, we observe that the performance indicator of both original and robust Black-Litterman strategies are very sensitive to the uncertainty of the views. Specifically, the original model perform poorly when the views are biased and well when the forecasters are generally correct. We also see that the robust models outperforms when the views are generally incorrect (i.e. with bias) and the performance gap becomes wider when the uncertainty parameter $\tau_{\mathbf{q}}$ increases. This insights may provide considerable benefits for investors that use the Black-Litterman model on their investment strategy.

\section{3}

\section{Sensitivity to Accuracy of the Forecasters}

In this section we test the point estimates for the classical BlackLitterman and our robust Black-Litterman models for different levels of accuracy on the views. The data used on the simulations are obtained from the data we simulate as described in section 4.1. Besides comparing the models on accuracy basis, we also check our insights for various levels of views's dispersion (i.e. $\left.\tau_{\mathbf{q}}\right)$. However, most of the results on the topic were presented on the previous section.

In figure 4.8 we investigate the empirical performance of our simulations. We also note that $\xi_{\mathbf{q}}=0$ is a turning point of our simulations, which is when robust models that consider views's dispersion start to perform worse. The reason is that, all of our hypothetical assets have positive expected return, thus, when the accuracy parameter $\left(\xi_{\mathbf{q}}\right)$ takes positive value the average view begins to capture the true direction of the returns. We would argue that this 
region is possibly the most realistic dynamic faced by practitioners, where market bets fluctuate between right and wrong directions. At this value of $\xi_{\mathbf{q}}$, we see a clear different of behavior on all statistical metrics.

An intuitive insight from this experiment is that, the relative advantage of the robust Black-Litterman strategies to the traditional one depends on both the precision of the views and its dispersion. From the Black-Litterman models that does not incorporate views's uncertainty structure (i.e. Average, Ellipsoidal $(q)$ and Ellipsoidal $\left(\mu_{B L}\right)$, we observe that the precision is only relevant factor for the performance. In fact, there is minimal impact on the average performance as $\tau_{\mathbf{q}}$ varies. This behavior, however, is not observed on other robust models. On these models we see that they do not tilt as much on the exposure of the precision parameter.
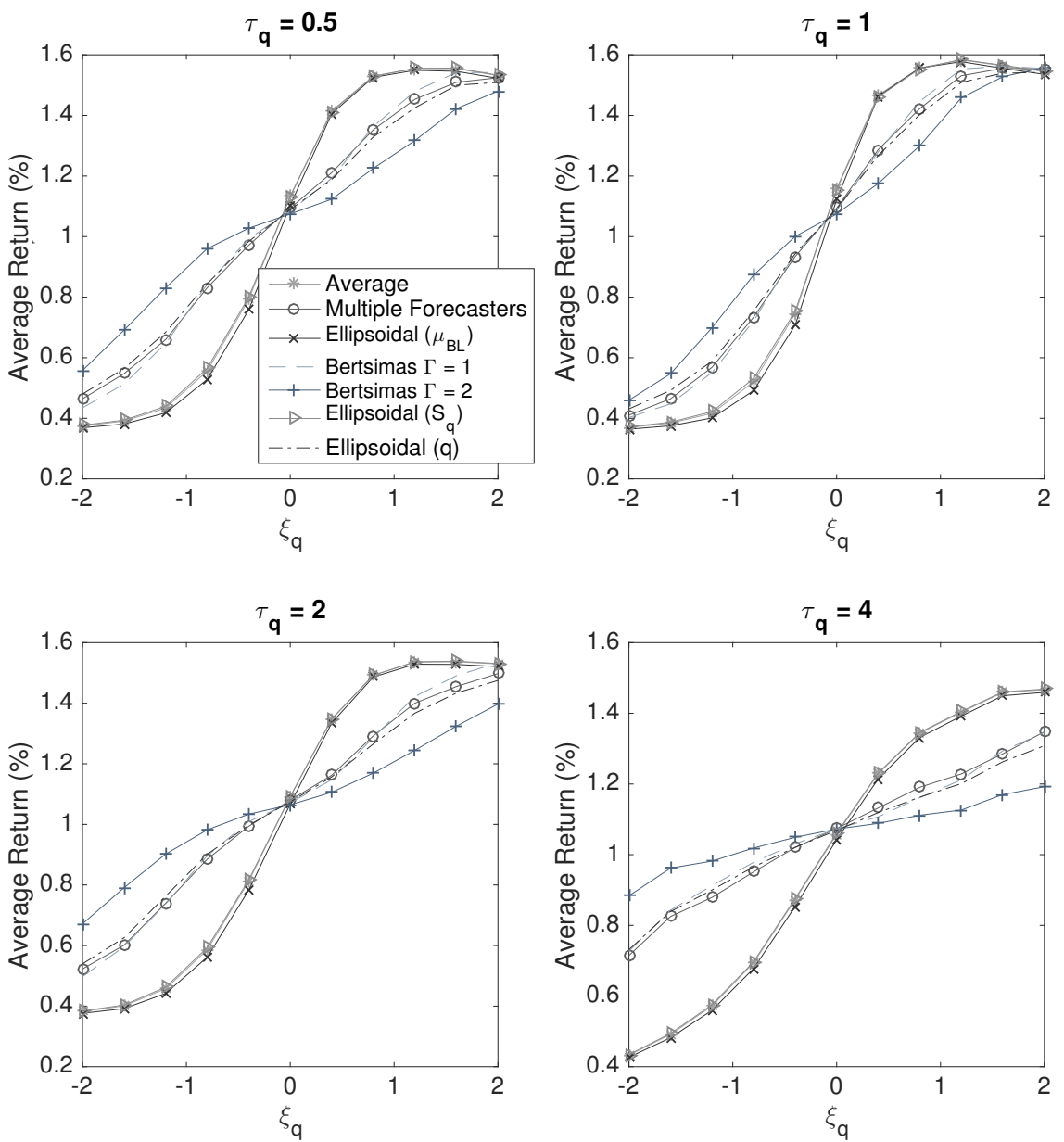

Figure 4.8: Average return of the simulations x $\xi_{\mathbf{q}}$

For Bertsimas and Sim, Multiple Forecasters and Elipsoidal $(q)$ we observe 
a similar pattern on the average returns. For these models the average return is not only a function of the accuracy factor $\xi_{\mathbf{q}}$, there is also a substantial part of the impact comes from the dispersion of the forecasters. In relative terms, we have that for $\xi_{\mathbf{q}}$ below zero the the average return is higher for greater levels of $\tau_{\mathbf{q}}$. On the other hand, we observe the opposite behavior when $\xi_{\mathbf{q}}$ is positive, the size of the uncertainty set has a negative contribution on the performance. This corroborates with the idea we have mentioned about the cost of robustness.
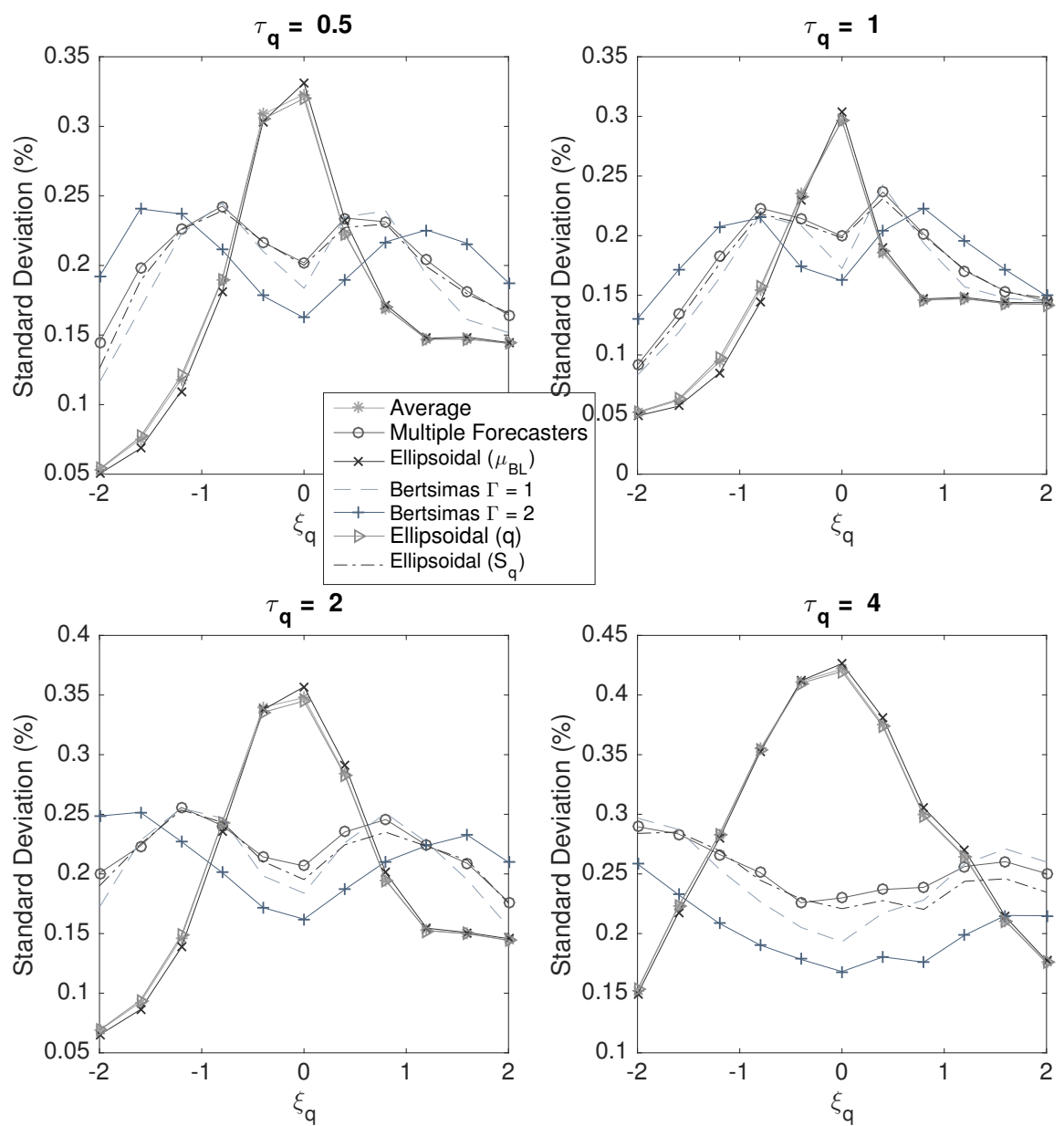

Figure 4.9: Standard deviation of the simulations $\times \xi_{\mathbf{q}}$

We plot in figure 4.9 the standard deviation of the simulations as a function of the accuracy parameter $\xi_{\mathbf{q}}$. Analogous to the results from the previous section, using the uncertainty structure of the views results on a more stable out of sample standard deviation. This general fact is observable in all considered robustifications that acknowledge the uncertainty structure, 
independently of the particular specification of the employed uncertainty set and degree of dispersion $\tau_{\mathbf{q}}$.

A more interesting finding is observed for values of $\xi_{\mathbf{q}}$ around zero. As we mentioned, this is a transition region of forecasts direction of the actual returns. In this region the original Black-Litterman model have a spike of volatility, which is due to the uncertainty around actual direction of the market's views. However, robust models have a smooth volatility transition around this region. These results empirically confirm an intuitive understanding that the robust strategy less sensitive to the accuracy of the views.
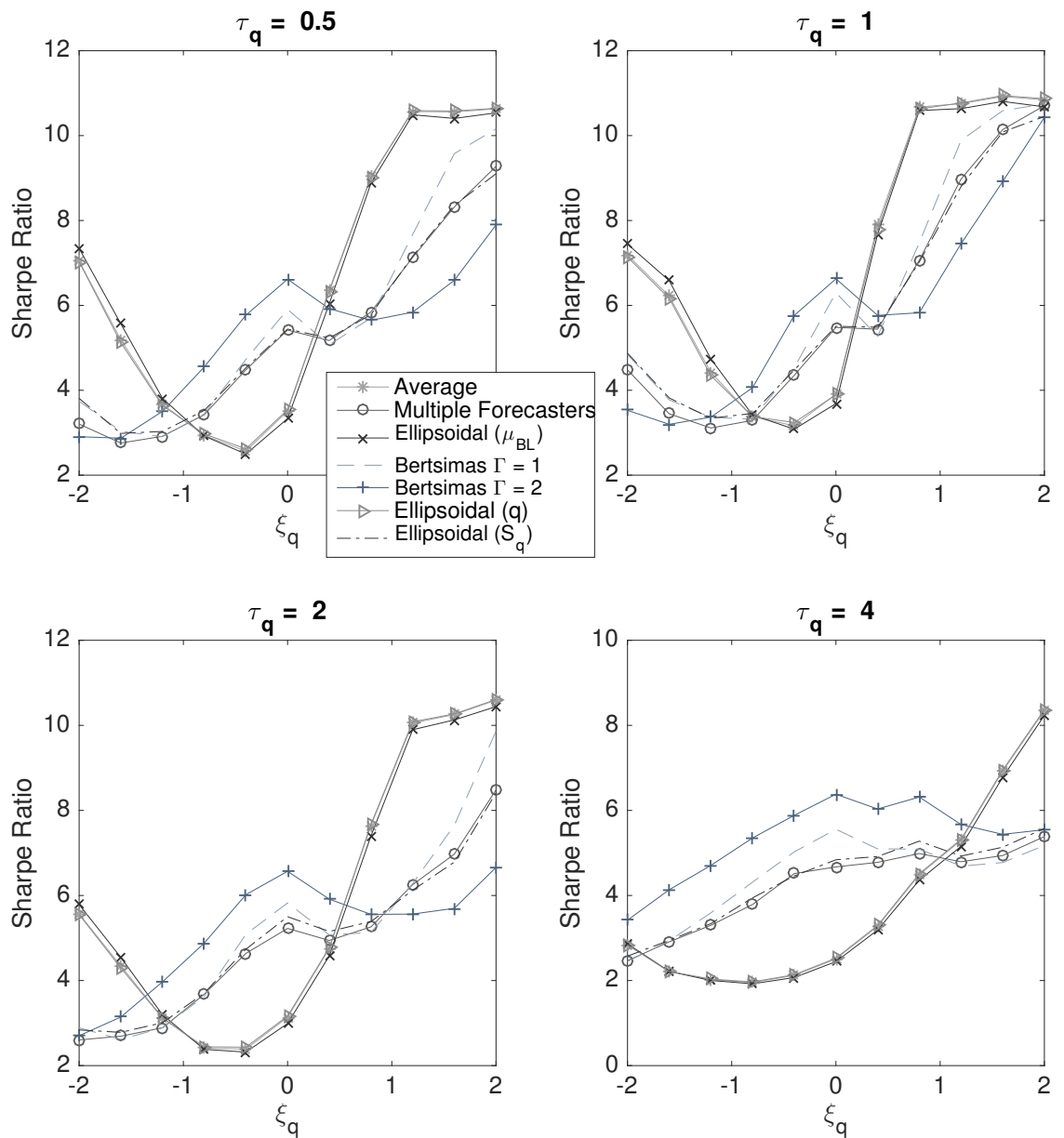

Figure 4.10: Sharpe ratio $\times \xi_{\mathbf{q}}$ 

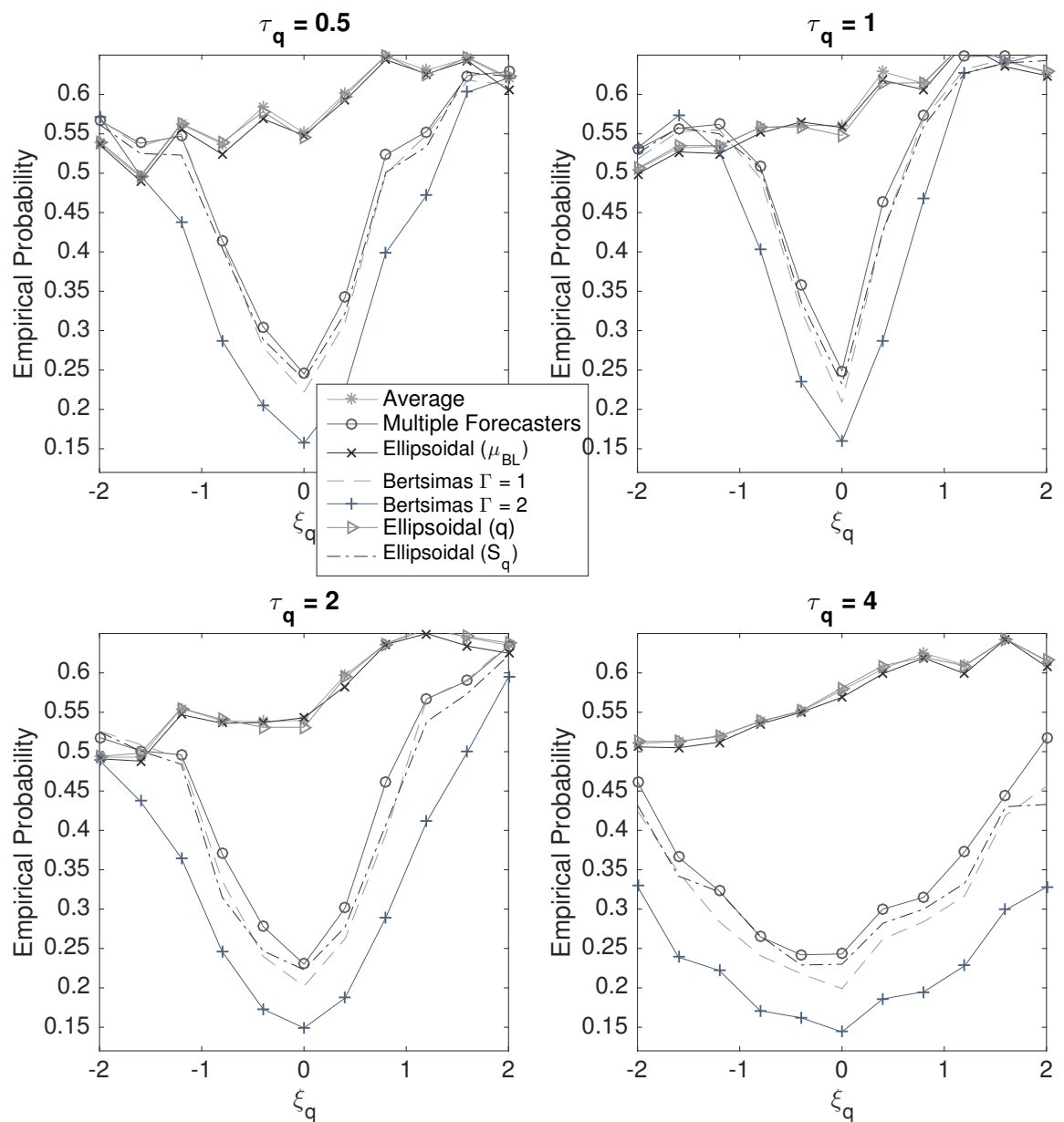

Figure 4.11: Empirical probability of the simulations $\times \xi_{\mathbf{q}}$

Figures 4.10 and 4.11 we plot the Sharpe ratio and empirical probability. These plots support the following observations:

(a) The robust strategies that consider the structure of the views have higher Sharpe ratios around $\xi_{\mathbf{q}}=0$ mark and lower ratios on extreme values of the parameter. For positive values of $\xi_{\mathbf{q}}$ it is mostly due to the better performance of the less robust models and the original Black-Litterman. Moreover, as the accuracy parameter assumes negative values the higher Sharpe ratio comes from the low volatility of these strategies, which actually have lower average return compared to the other models;

(b) The empirical probability is significantly dependent on the level of robustness. For larger uncertainty sets we observe a lower empirical probability in the results of figure 4.11. For example, we note that the empirical prob- 
ability of the most robust model Bertsimas with $\Gamma=2$ lower bounds all other models on most part of our simulations. We also observe a reduction of the empirical probability around $\xi_{\mathbf{q}}$ equal zero and as $\tau_{\mathbf{q}}$ increases.

The results presented in this section demonstrate several important points. First, our argument of the importance of considering a robust structure on the views of the Black-Litterman model. As we have show empirically, its influence can substantially effect both the standard deviation and average return of the portfolio. Second, our observations have shown that increasing the robustness of views decrease the performance dependency on the accuracy of the forecasts. Also, we have seen that the robust formulation has a cost when the forecasters are in general correct, a price to pay to be insured on multiple market views. Moreover, the robustness effect becomes more apparent when multiple forecasters are uncertain about the direction of the returns, which is the case for $\xi_{\mathbf{q}}$ equal to zero. Finally, To restate the computational tractability of our robust formulation, we generate a fictitious problem with 100 risky assets and 100 absolute views. This large scale problem was solved in $0.4 \mathrm{~s}$ and $0.8 \mathrm{~s}$ for Bertsimas and Ellipsoidal uncertainty sets respectively, which is an acceptable time for most practical portfolio problems. 


\section{5}

\section{Market Expectations System Backtest}

In this case study we analyze the performance of two proposed models (Bertsimas approach and ellipsoidal uncertainty) in an out-of-sample exercise applied to the Brazilian financial market data considering a hedge fund strategy. The views were modeled using the Market Expectations System, which was developed by the Central Bank of Brazil to collect macroeconomic projections from the industry. We divided this chapter in four parts. First, we explain what is the Market Expectations System. Second, we present the assumptions for the backtests and data sets that used throughout our studies. Then, we explain how we modeled the views on fixed income securities. Finally, we present the results from the backtests. The backtests were conducted in Julia Language using JuMP for mathematical optimization (Dunning et al., 2015).

\section{1 \\ Market Expectations System}

Here we briefly describe the Market Expectations System developed by the Central Bank of Brazil to aid in its monetary policy decisions. For more details on the platform we recommend Barbosa (2012).

The platform started in May 1999, as an online tool, where projections are input by institutions working in the financial market, such as banks, asset managers and consultants. In other cases, th are also added companies that have specialized teams who produce forecasts concerning the main macroeconomic variables. Its main objective is to assist the decision making by professionals of the Central Bank itself as well as other enterprises and citizens who may use the data to plan their actions. The projections are performed for variables related to economic activity, interest rates and exchange rates, the variation of price indices, the balance of payments and the fiscal sector of the Brazilian economy. However, the individual information of the participants are confidential and it is only is available to the Brazilian Monetary Policy Committee (COPOM) and the managers of the system itself.

Currently, there are around 130 logins active in the system. For statistics purposes, the system only considers the data provided in the last 30 days. Therefore, the system automatically disregards its projections when calculating the daily statistics, if the institution does not update its forecast in a frequency 
of at least 30 days. The statistics generated by the system are available to the public in a daily basis by the Central Bank, where they provide the median, average, standard deviation, coefficient of variation, maximum and minimum. Also, the Brazilian Central bank releases on a weekly basis Focus Market readout with a summary of these statistics. The projections can be done in a yearly, monthly or quarterly basis, depending on the macroeconomic variable. The Central Bank also ranks the top five institutions by their forecasts, classifying based upon their accuracy in short, medium and long term.

In this work we are only interested the projections of three macroeconomic variables, the Selic Rate ${ }^{1}$, Consumer Price Index (IPCA) and US Dollar exchange rate. Their reference dates (i.e. day of the prediction) are:

1. US Dollar Exchange Rate: there are two references date for the exchange rate forecast, the last day of the previous month and last business day before or on the 15th of the current month;

2. Selic rate: last business day before or on the Wednesday of the week preceding the Brazilian monetary policy committee meeting of the reference period and the last business day before or on the 4th Wednesday preceding the COPOM meeting of the reference period. For months with no meeting the forecasts are not registered in the system;

3. IPCA: last business day before IPCA-15 is released;

Statistics generated from these variables are inputs to model views in the Black-Litterman. Since it is not provided the forecast of each institution, we do not use the Robust Model with multiple forecasts.

\section{2}

\section{Interest Rate Forecast to Returns}

In this section we present how to price a government bond. Then, we briefly introduce the dynamics of interest rate term structure based on expectation hypothesis and how we used the forecasts on the spot rate to make views for the Black-Litterman model.

Bond portfolios are vulnerable to a considerable number of risk factors. However, for government fixed and index-linked bonds, Koivu and Pennanen (2014) show most of monthly portfolio returns are consistently explained by the yield to maturity and the underlying index. Which reduces the return

\footnotetext{
${ }^{1}$ Selic rate is an average of the interbank interest rates which are charged for the trade in government securities with a maturity of 1 day. Since March 1999, the Central Bank of Brazil is using this interest rate as benchmark short-term interest rate for its monetary policy.
} 
uncertainty into one and two factors, for further details see Fabozzi and Mann (2011); Fabozzi (2012); Koivu and Pennanen (2014). Here we explain how to use the interest rate and inflation predictions to forecast returns for government bond securities.

Fixed income security can be divided in two different groups, zerocoupon bonds, also known as discount bonds and coupon bonds. The difference between them is how the payment is delivered to its buyer, zero-coupon bonds makes a single payment at the maturity, whereas coupon bonds makes regular interest payment that are predetermined in the purchase and the last compensation is due at its maturity. Fabozzi and Mann (2011) defines a coupon bond as a set cash flows, that each redemption payment is equivalent to a zero coupon bond maturing at its respective date.

Consider a general portfolio of bonds with payments that is due at times $t_{1}, t_{2}, \ldots, t_{N} \in \mathbb{R}^{+}$. We denote the nominal payment as $C_{t_{n}} \in \mathbb{R}^{+}$, forward rate from $t$ to $t_{n}$ and linked index at time $t$ as,$Y_{t, t_{n}} \in \mathbb{R}$ and $I_{t} \in \mathbb{R}$ respectively. Thus, the bond portfolio market price, $P_{t} \in \mathbb{R}^{+}$, at $t<t_{1}$ is given by

$$
P_{t}=\sum_{n=1}^{N} \frac{I_{t} C_{t_{n}}}{\left(1+Y_{t, t_{n}}\right)^{\left(t_{n}-t\right)}},
$$

for continuous compounded interest rate the price is defined as

$$
P_{t}=\sum_{n=1}^{N} \exp \left(-y_{t, t_{n}}\left(t_{n}-t\right)\right) I_{t} C_{t_{n}} .
$$

The relation between continuously compounded rate and discrete rate is denoted as

$$
y_{t, t_{n}}=\ln \left(Y_{t, t_{n}}+1\right)
$$

For inflation-indexed bonds, $I_{t}$ is the consumer price index, which in Brazil is called IPCA and $Y_{t, t_{n}}$ is the real yield. In case of fixed income securities, there is no indexed cash flow, meaning that $I_{t}$ always takes value one and $Y_{t, t_{n}}$ is the nominal interest rate. Observe that the nominal payments is not a function of $t$, it is the future cash flow to be received at $t_{n}$.

\subsection{1}

\section{Interest Rate Curve Dynamics}

There are many empirical studies in the literature that aims to identify the expected return relationships between bond securities across time. According to Campbell et al. (1997), the expectations hypothesis is the most popular model of the term structure of interest rate. The theory determines a relationship between long term and short term interest rates. For a more thorough discussion in this subject, see Luenberger (1998), Campbell et al. (1997) and 
Tabak and Andrade (2001). To simplify, in the remaining of this section we discuss these concepts assuming zero-coupon bonds, however, it could also be generalized for coupon bonds.

The underlying idea of this method is that the implied forward rates from the current yield curve will be realized. In other words, long term bonds are defined as the expected future spot rates. One can also interpret that it assumes zero expected excess return on long-term bonds over short-term bonds. Under these assumptions we can imply future spot rates and forward rates from the current yield curve. In discrete time, one form to represent this hypothesis is assuming that a $n$-period bond return is a combination of the market's expectations for $m \tau$-period short interest bond. We can express it as the following:

$$
\left(1+Y_{t, t_{n}}\right)^{n}=\mathbb{E}\left[\left(1+Y_{t, t+\tau}\right)^{\tau}\left(1+Y_{t+\tau, t+2 \tau}\right)^{\tau} \ldots\left(1+Y_{t+(m-1) \tau, t_{n}}\right)^{\tau}\right],
$$

where $t_{n}=t+m \tau$ and the left side of the equality is the return guaranteed when buying the bond at time $t$ and holding until maturity at $t_{n}$.

In table 5.1 we illustrate these

A second form of the expectation hypothesis states that a $\tau$ period ahead expected return reflects the expectations on the forward rate from $\tau$ to $t_{n}$

$$
\mathbb{E}\left[\left(1+Y_{t, t+\tau}\right)^{\tau}\right]=\left(1+Y_{t, t_{n}}\right)^{n} \mathbb{E}\left[\left(1+Y_{t+\tau, t_{n}}\right)^{\tau-n}\right] .
$$

Although equations 5-5 and 5-4 represents different forms of the expectation hypothesis in discrete time, they are not equivalent whenever the interest rate are random. There are two issues in assuming that both equations are true. First we would have to make another assumption that the forward rates $\left(1+Y_{t+j \tau, t+(j+1) \tau}\right)^{\tau} \forall j=0, \ldots, m-1$ are independent. Also, the expected value of a random variable is not necessarily the inverse of its expected value.

Due to these issues, it is common to work with log returns of compounded interest rate random variables, which has the benefit of linearizing exponential affine functions. When the expectation hypothesis are formulated in this manner, all forms of expectation hypothesis become equivalent. Furthermore, it becomes possible to define comparable forward rates for independent values of the investment horizon $\tau$. Using $\log$ returns, the counterpart of equation 5-5 becomes

$$
\mathbb{E}\left[r_{t, t+\tau}\right]=\left(y_{t, t_{n}}-\mathbb{E}\left[y_{t+\tau, t_{n}}\right]\right) n+\mathbb{E}\left[y_{t+\tau, t_{n}}\right] \tau,
$$

where $r_{t, t+\tau}$ defines the log return from time $t$ to $t+\tau$. Moreover, for zerocoupon inflation-linked bonds we have the equivalent log return formulation 


$$
\mathbb{E}\left[r_{t, t+\tau}\right]=\left(y_{t, t_{n}}-\mathbb{E}\left[y_{t+\tau, t_{n}}\right]\right) n+\mathbb{E}\left[y_{t+\tau, t_{n}}\right] \tau+\mathbb{E}\left[\pi_{t, t+\tau}\right] \tau,
$$

where $\pi_{t, t+\tau}$ is the continuously compounded rate of inflation. The views returns on fixed-income securities are modeled using equations 5-6 and 5-7. In the following example we illustrate how to use forecasts on spot rates to generate return views on fixed rate bonds.

In this example we compare the implied SELIC rates from the current Brazilian Yield curve with forecasts predicted by market practitioners available on the Market Expectations System. We use the short term top five institutions. The implied SELIC rates are assumed as the forward rates between COPOM meetings, which are the dates when Brazil's Central bank announces a possible change on the current SELIC rate. To model the yield curve, we use data from fixed-float rate swap contracts of 02/07/2017. These contracts are traded in the Brazilian Mercantile and Futures Exchange (BM\&F Bovespa) on business days according to the Brazilian calendar.

Table 5.1 displays the yield curve data and the forecasts on the interest rate from the Market Expectations System. We detone $t_{0}$ as the current yield curve date $(02 / 07 / 2017), t_{1}, \ldots, t_{n}$ as the COPOM meetings dates and the annualized interest rates are all in percentage. The maximum, average and minimum forecasts are denoted as Max F., Mean F. and Min F. respectively.

\begin{tabular}{c|c|c|c|c|c|c}
\hline \hline COPOM $\left(t_{n}\right)$ & $Y_{t_{0}, t_{n}}$ & $Y_{t_{n}, t_{n+1}}$ & $\Delta Y_{t_{n}, t_{n+1}}$ & Max F. & Mean F. & Min F. \\
\hline \hline $2 / 23 / 2017$ & 12.88 & 12.08 & -0.80 & 12.25 & 12.25 & 12.25 \\
\hline $4 / 13 / 2017$ & 12.30 & 11.39 & -0.68 & 11.50 & 11.50 & 11.50 \\
\hline $6 / 1 / 17$ & 11.92 & 10.83 & -0.56 & 11.00 & 10.89 & 10.75 \\
\hline $7 / 27 / 17$ & 11.55 & 10.40 & -0.44 & 10.50 & 10.36 & 10.00 \\
\hline $9 / 8 / 17$ & 11.32 & 10.08 & -0.32 & 10.00 & 9.86 & 9.50 \\
\hline $10 / 26 / 17$ & 11.09 & 9.87 & -0.21 & 10.00 & 9.64 & 9.50 \\
\hline $12 / 7 / 17$ & 10.92 & 9.75 & -0.11 & 10.00 & 9.57 & 9.25 \\
\hline $1 / 11 / 18$ & 10.80 & 9.70 & -0.05 & 10.00 & 9.58 & 9.25 \\
\hline $2 / 22 / 18$ & 10.68 & 9.68 & -0.02 & 10.00 & 9.54 & 9.25 \\
\hline $4 / 12 / 18$ & 10.56 & 9.69 & 0.01 & 9.75 & 9.50 & 9.00 \\
\hline $5 / 24 / 18$ & 10.39 & 9.72 & 0.03 & 9.75 & 9.33 & 9.00 \\
\hline $7 / 12 / 18$ & 10.41 & 9.78 & 0.05 & 9.75 & 9.33 & 9.00 \\
\hline
\end{tabular}

Table 5.1: Comparison of implied SELIC rates and its forecasts from the Market Expectations system.

The expected returns for a one year government bond for an investment 
horizon of 21 business days is presented in table 5.2. As expected, we see the analysts forecasting a higher spot rate a year from now results in a lower estimated return. On the other hand, the average and minimum forecasts are expecting a higher return than what is already priced in the yield curve.

\begin{tabular}{c|c|c|c|c}
\hline \hline & Market & Max F. & Mean F. & Min F. \\
Expected Return & $0.85 \%$ & $0.73 \%$ & $0.88 \%$ & $1.04 \%$ \\
\hline \hline
\end{tabular}

Table 5.2: Expected return using the Market Expectations system as views

Using the forecasts, figure 5.1 shows that the implied curves and the actual yield curve are very close. However, the gap between the maximum and minimum yield forecasts increases with maturity, reinforcing the need of optimization under uncertainty for allocation decisions.

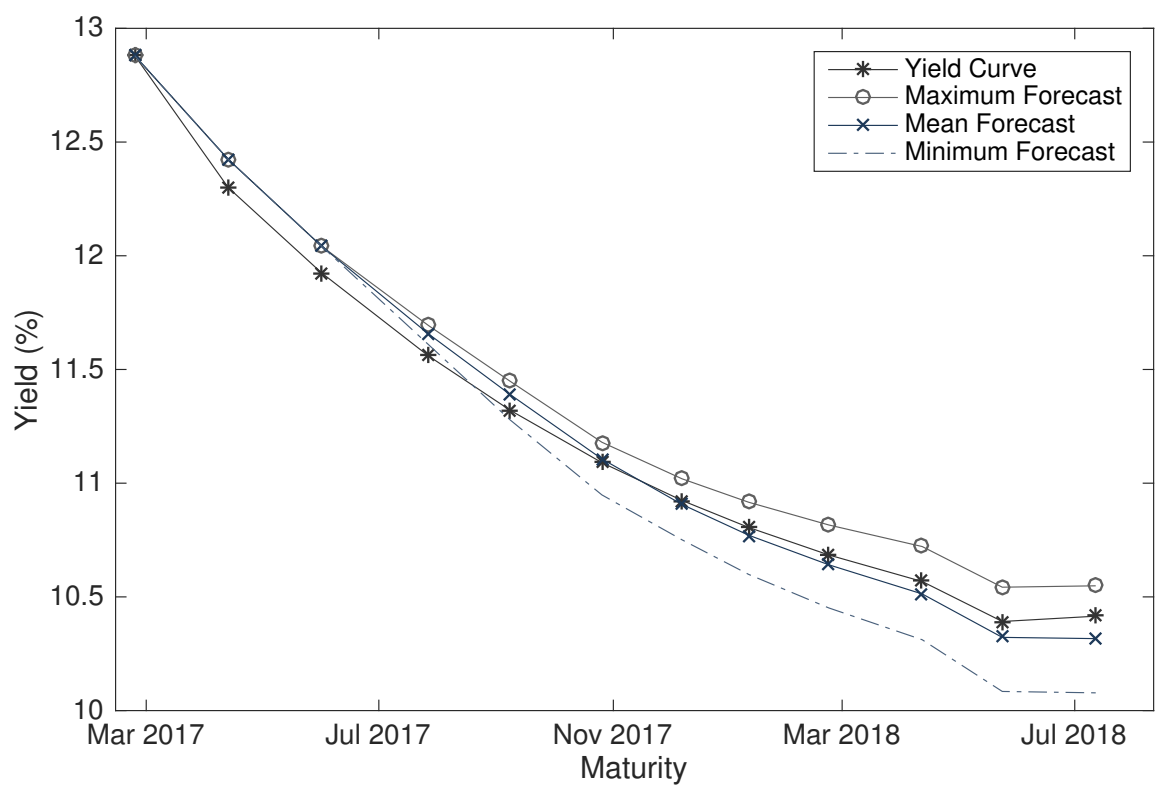

Figure 5.1: Implied yield curves from the forecasts and the nominal yield curve at $02 / 07 / 2016$.

\section{3}

\section{Data and Assumptions}

We consider daily data set ranging from March '09 to October '16, which is the total data available from the market expectations system top five forecasters at the time this test was conducted. We start our investment in March '10 and use one year of historical data to estimate the covariance 
matrix. The investor rebalances his portfolio on a monthly basis. Portfolios are modeled with a target annualized standard deviation of $6 \%$. In addition, leverage and short selling are not allowed. The universe of assets are set as the following asset classes

- U.S Dollar (BRLUS);

- Bovespa Index (IBOV);

- Brazilian fixed income bonds with constant duration of 2 years (iDkA PRE 2Y);

- Brazilian inflation-linked bonds with constant duration of 2 years (iDkA - IPCA 2Y);

- Risk-Free asset: Interbank Deposit Rate $(\mathrm{CDI})^{2}$;

The cumulative return of these assets in the period of tests is presented in figure 5.2

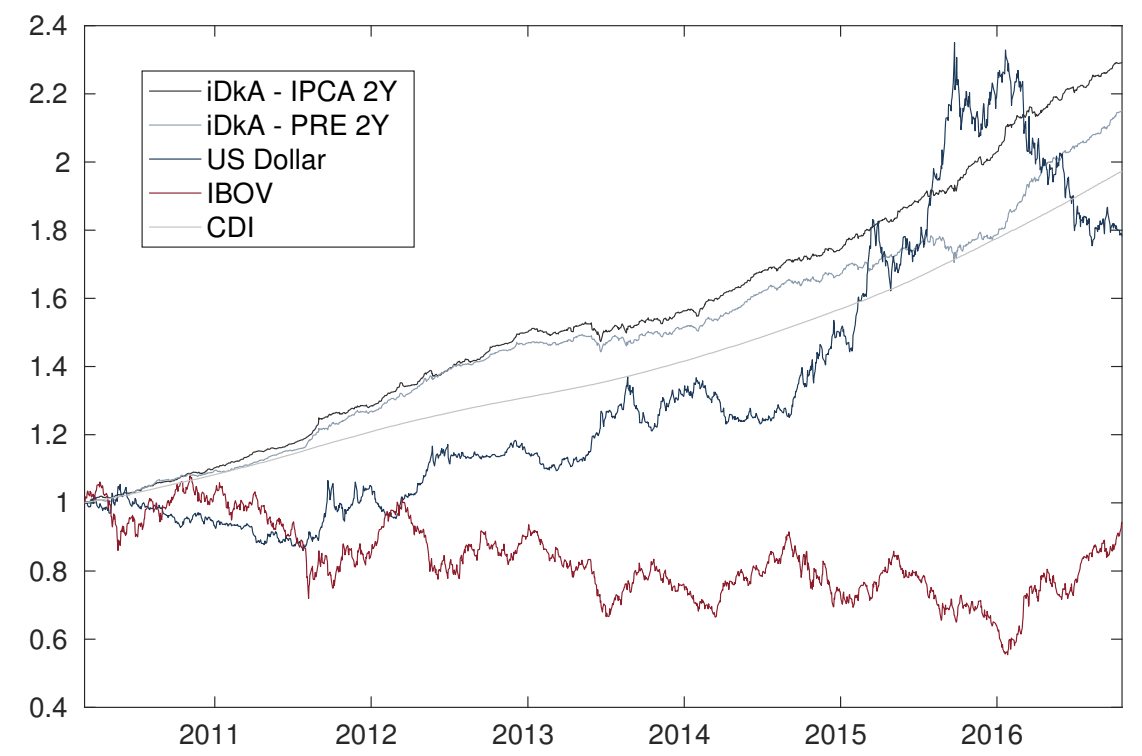

Figure 5.2: Cumulative returns of the asset classes from March 2010 until October 2016

${ }^{2}$ The Interbank Deposit Rate (CDI) is the overnight rate which a financial institution lends funds to another financial institution in the overnight market. The overnight rate is the lowest available interest rate used by Brazilian financial institutions to manage liquidity and satisfy norms and regulations. It is the most common risk-free benchmark used by hedge fund in the Brazilian financial market. 
As can be observed in figure 5.2, on our period of analysis the Brazilian stock market goes through a period of bear market, resulting in a negative performance of the stock index.

We opted to use fixed income securities using constant duration. According to Meucci (2005) this is the most convenient representation of the timehomogeneous invariants (i.e. Distribution independent of a reference time $t$ ) for the fixed-income market. To represent these securities we use the Index of Constant Duration Anbima (IDkA), which is a series of synthetic indexes to represent Brazilian government bonds with constant duration.

The assumptions of the Black-Litterman model go as follows

- Market Assumptions

1. We consider the following for the capitalization weights for the CAPM

(a) U.S Dollar: Total investment abroad by Brazilian citizens reported by the Brazilian Central Bank;

(b) Bovespa Index: Total market value of the companies that compose the index;

(c) Brazilian fixed-rate bonds: Total national debt issued by the Brazilian government with fixed-rate bonds;

(d) Brazilian inflation-indexed bonds: Total national debt issued by the Brazilian government with inflation-indexed bonds;

In figure 5.3, we illustrate the market portfolio from September 2003 to October 2016 


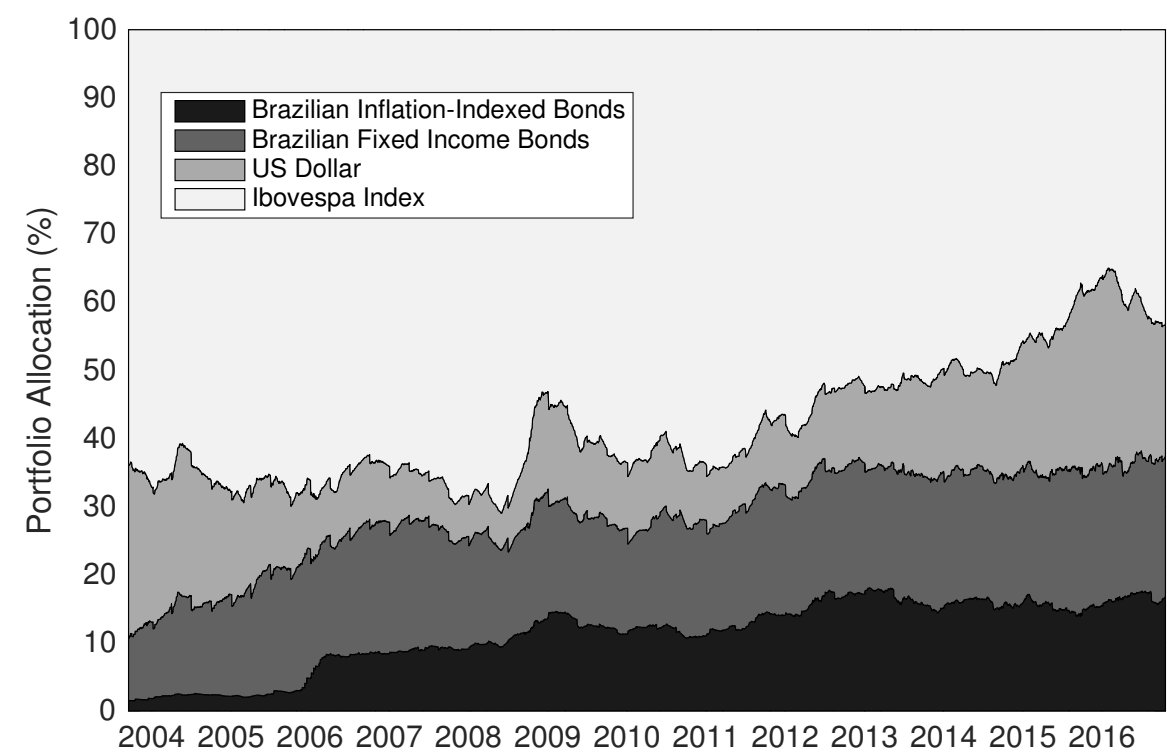

Figure 5.3: Market portfolio from September 2003 to October 2016.

2. Parameter $\tau$ is set 0.05 as in He and Litterman (1999).

- Views Assumptions

1. We consider absolute views on U.S. Dollar, fixed-rate bonds and inflation indexed bonds, therefore

$$
\left[\begin{array}{c}
q_{I P C A} \\
q_{S E L I C} \\
q_{D O L}
\end{array}\right]=\left[\begin{array}{llll}
1 & 0 & 0 & 0 \\
0 & 1 & 0 & 0 \\
0 & 0 & 1 & 0
\end{array}\right]\left[\begin{array}{c}
\mu_{I P C A} \\
\mu_{S E L I C} \\
\mu_{D O L} \\
\mu_{I B O V}
\end{array}\right]+\left[\begin{array}{c}
\epsilon_{1} \\
\epsilon_{2} \\
\epsilon_{3}
\end{array}\right],
$$

2. All views are done using the short-term top five institutions from the Market Expectations system. For each uncertainty set we consider the following inputs:

(a) Bertsimas and Sims uncertainty sets: average, maximum and minimum values;

(b) Ellipsoidal uncertainty sets: average and five forecasters. Since there is no information about the dispersion of the forecasters we only use the first two models presented in this work.

3. The confidence matrix is parametrized by parameter $\tau_{c}$, which is used to analyze how the confidence in the forecasters affects the 
overall portfolio results. Therefore, the confidence matrix is chosen as $\boldsymbol{\Omega}=\mathbf{P}(\tau \hat{\mathbf{\Sigma}}) \mathbf{P}^{\prime} / \tau_{c}$, where we set $\tau_{c}=(0.01,1,10,100)$.

\section{4 \\ Results}

We now present the out of sample results of the portfolio weights using different robust strategies on the Black-Litterman model. Our analysis are done in two steps. First, compare the performance of all strategies. To better isolate the effects of the uncertainty set and accuracy from the data base, we proceed our studies contrasting the models for different levels of confidence on the views. Second, we study the effect of the uncertainty sets on portfolio allocation.

In table 5.3 we calculate the mean return and standard deviation experienced for all strategies employed over the backtests. It can be observed that the performance on both the classical and the robust portfolio increases as the confidence parameter $\tau_{c}$ assumes higher values. Therefore, it suggests that macroeconomic forecasts on the Market Expectation system data base adds values to the portfolio allocation. Further in this section we analyze periods where the forecasts were more effective.

\begin{tabular}{cccccccc}
\hline \hline$\tau_{c}$ & & $\mathrm{~B}(\Gamma=2)$ & $\mathrm{B}(\Gamma=1)$ & $\mathrm{EL}\left(\mu_{B L}\right)$ & $\mathrm{EL}(q)$ & Av. BL & CAPM \\
\hline \hline 0.1 & Return & 8.55 & 8.35 & 7.97 & 8.18 & 8.32 & 8.28 \\
& Standard Dev. & 6.18 & 6.17 & 6.22 & 6.17 & 6.15 & 6.26 \\
\hline \multirow{2}{*}{1} & Return & 9.11 & 8.83 & 8.66 & 8.02 & 8.28 & 8.28 \\
& Standard Dev. & 6.33 & 6.34 & 6.36 & 6.36 & 6.33 & 6.26 \\
\hline \multirow{2}{*}{10} & Return & 9.09 & 9.26 & 8.98 & 8.63 & 8.78 & 8.28 \\
& Standard Dev. & 6.41 & 6.39 & 6.38 & 6.41 & 6.41 & 6.26 \\
\hline \multirow{2}{*}{100} & Return & 10.00 & 9.70 & 8.97 & 8.80 & 8.84 & 8.28 \\
& Standard Dev. & 6.18 & 6.25 & 6.38 & 6.42 & 6.42 & 6.26 \\
\hline
\end{tabular}

Table 5.3: Annualized average return and standard deviation from all strategies. All results are shown in percentage.

Across strategies, we note that Bertsimas with $\Gamma=1$ and $\Gamma=2$ have the best overall performance for all values of $\tau_{c}$, including the reference market model (CAPM). Between these two models we observe that $\Gamma=2$ is only outperformed when $\tau_{c}=10$. These numerical results suggests that robustification on the views of the Black-Litterman approach can really lead to an added value in asset management, as it can incorporate more information on views of asset returns. 
The same performance is not observable in the robustifications that only considers the average value of the views. The case of using confidence ellipsoid on $\mu_{B L}$ the perfomance is slightly above the original Black-Litterman, the exception here is when we assume the lowest confidence on the views (i.e. $\left.\tau_{c}=0.1\right)$. For Ellipsoidal $(q)$, the performance is always below the average Black-Litterman, however, it is still higher than the CAPM when $\tau_{c}$ equals to 10 and 100.

For standard deviation, we observe that both the classical and the robust Black-Litterman models underestimate the volatility for the portfolios. Therefore, a higher volatility are actually realized on the out of sample results. To closely examine the characteristics of the out of sample returns, in figure 5.4 we show the boxplots of a total 1669 daily out of sample returns for all allocation methodologies for $\tau_{c}$ assuming values of 1 and 100 .
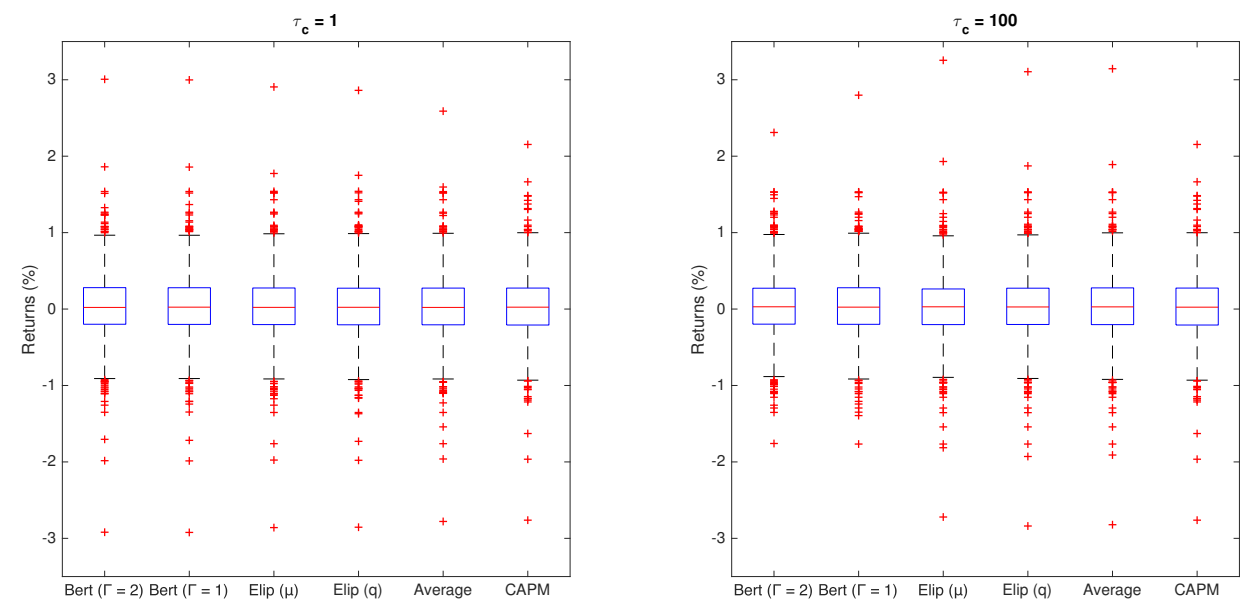

Figure 5.4: Boxplots of the out of sample daily returns for all allocation methodologies using $\tau_{c}=1$ and $\tau_{c}=100$.

The boxplots of the different portfolio allocation strategies give a graphical representation of the impact of both the confidence parameter and uncertainty sets on the out of sample returns. In the case of ellipsoidal uncertainty sets, the results look very similar to the average Black-Littermann. This result is expected, since the standard form of the original Black-Litterman considers that the uncertainty on both the posterior average return $\mu_{B L}$ and views $q$ are small, as we have mentioned chapters. Estimation of the Black-Litterman parameters are out of the scope of this thesis, however, one might use the statistical techniques presented in Allaj (2013) to estimate the Black-Litterman parameters and combine with our robust model. This robust approach might be useful for practitioners that use the Black-Litterman as an allocation model. 
We now discuss the boxplots of the daily returns using the strategies based on the Bertsimas and Sim's uncertainty set. It is clear from figure 5.4 that when the confidence on the views increases the worst-case scenario captured on the uncertainty set reflects on the portfolio allocation. We see that the uncertainty structure available on the views actually helps to mitigate possible downsides that is not captured when we only use the average. As a result, we observe that the worst daily returns seen for $\tau=1$ is not repeated when the confidence parameter increases to 100. However, the robust trade-off is also observed for the positive daily returns which has its maximum lower compared to other models. This results show that the average Black-Litterman is more dependent on the realization of the views, which corroborates with our empirical analysis using synthetic data.

The difference in out of sample returns we have discussed can be nicely seen in the cumulative historical performance illustrated in figure 5.5. All optimization methodologies attempt to realize the view by adjusting the estimate of the mean return away from the CAPM market equilibrium assumption. Consequently, the portfolios are very correlated and the performance are similar, however, when $\tau_{c}$ increases we have that the adjustment tilts heavier towards the views (i.e. a higher confidence on the forecasters). We note that the overall cumulative performance improved from the CAPM benchmark for all models when we use higher values of $\tau_{c}$. 

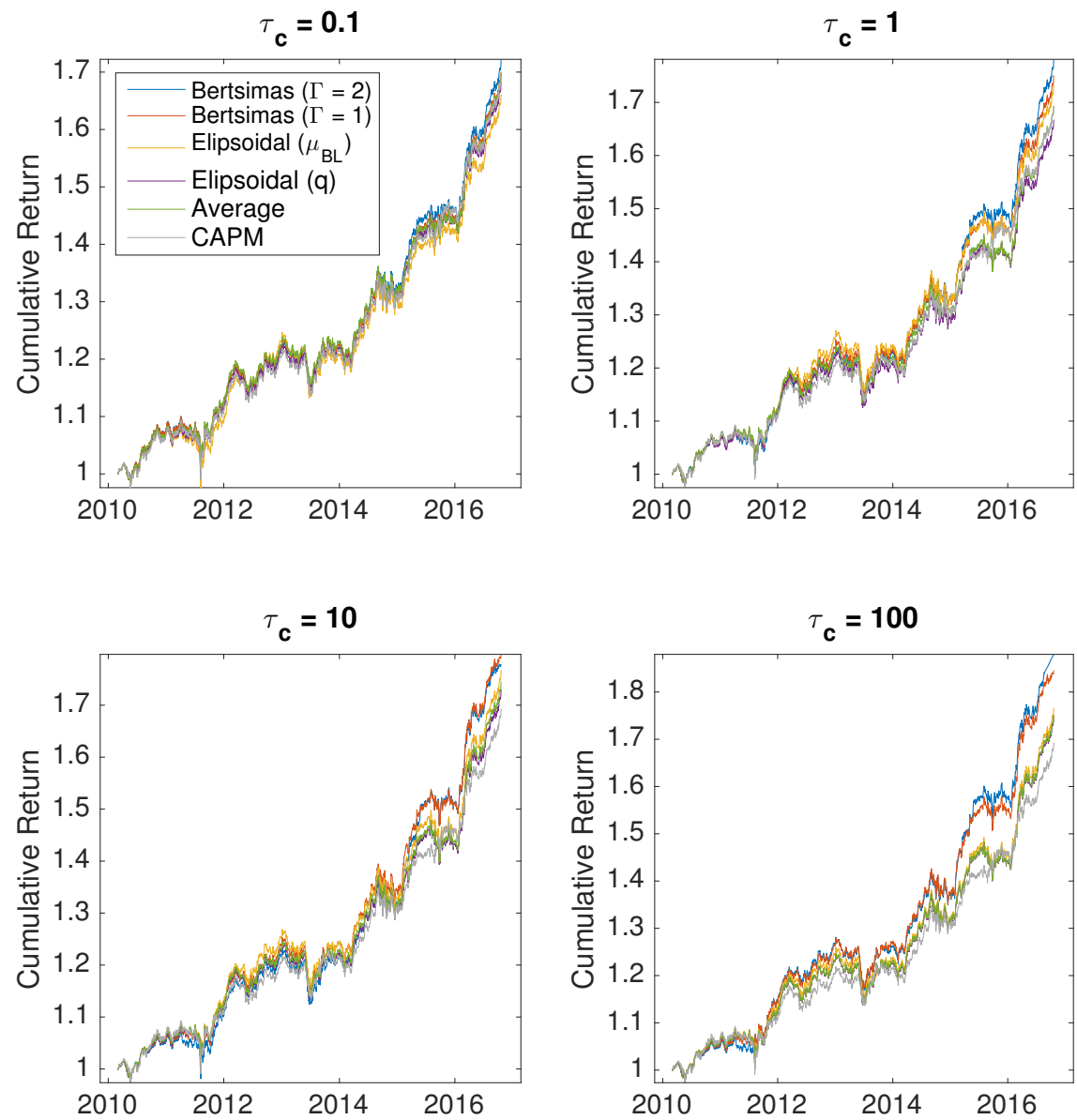

Figure 5.5: All strategies cumulative return for $\tau_{c}=(0.1,1,10,100)$.

We can also see that in the first half of our data period Bertsimas and Sim's uncertainty lead to moderately better out of sample results. Later on, towards the end of the time period all assets classes went through periods of bear and bull markets, mostly due to the Brazilian financial recession on the years of 2014 and 2015 and beginning of recovery in 2016 (see figure 5.2). The results in the second half showed that these robust models benefited from the uncertainty sets on the views throughout the different market phases.

We now turn to discuss our insights on the allocations of the robust methodologies constructed using different information on the views. Figures 5.6, 5.7, 5.8 and 5.9 plots the time-varying portfolio weights for each allocation approach and different values of $\tau_{c}$. Notice that the CAPM equilibrium is more of a passive strategy with a set target volatility and the Black-Litterman models actively tilts the allocations according to market expectations. There- 
fore, we can check that for lower confidence on the views the relative stability of all portfolios are higher, deviating less from the CAPM equilibrium. On the other hand, higher confidence impose heavier weights on directional views resulting in less stable monthly portfolios.
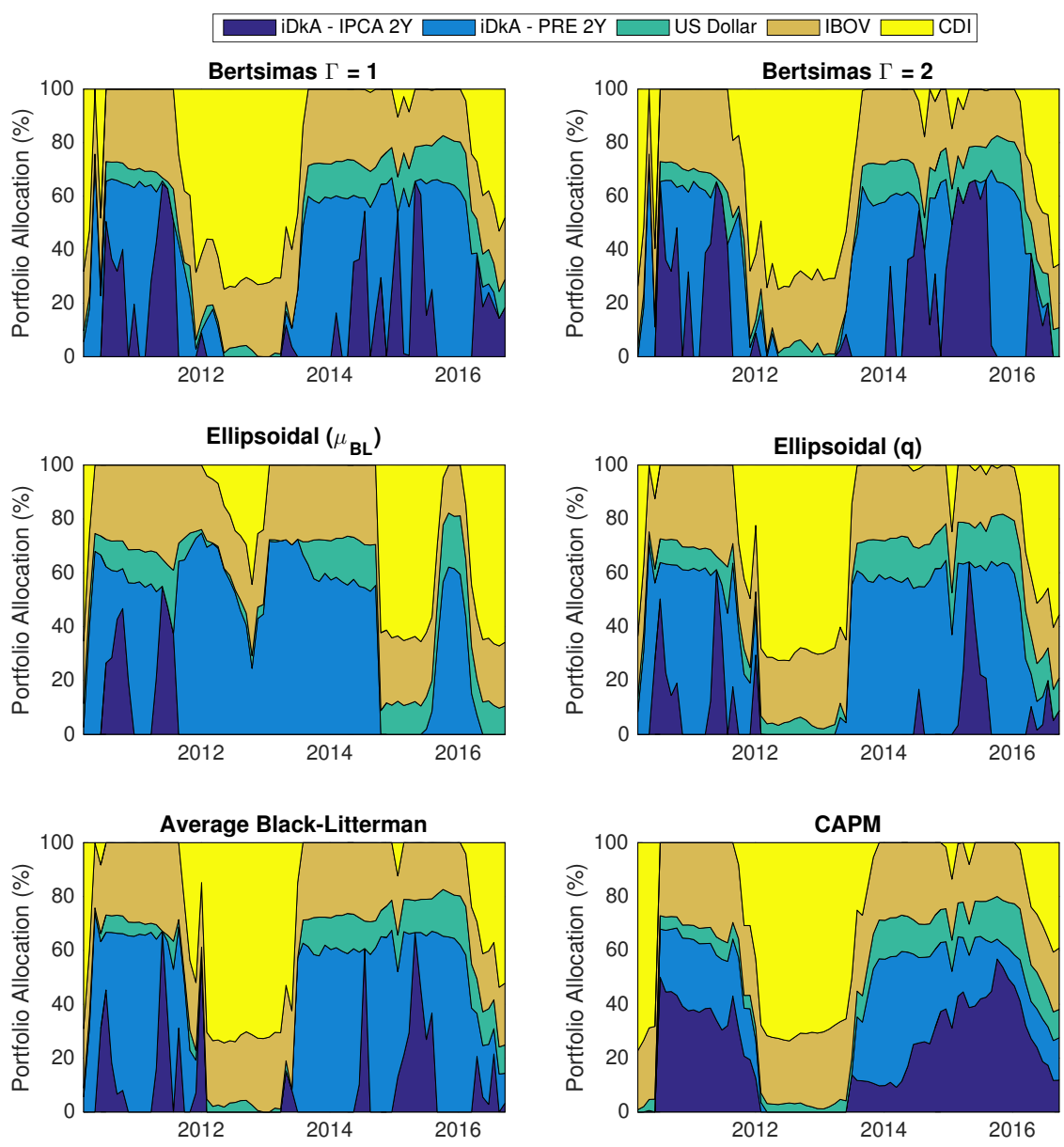

Figure 5.6: Portfolio weights in the period of analysis for $\tau_{c}=0.1$. 

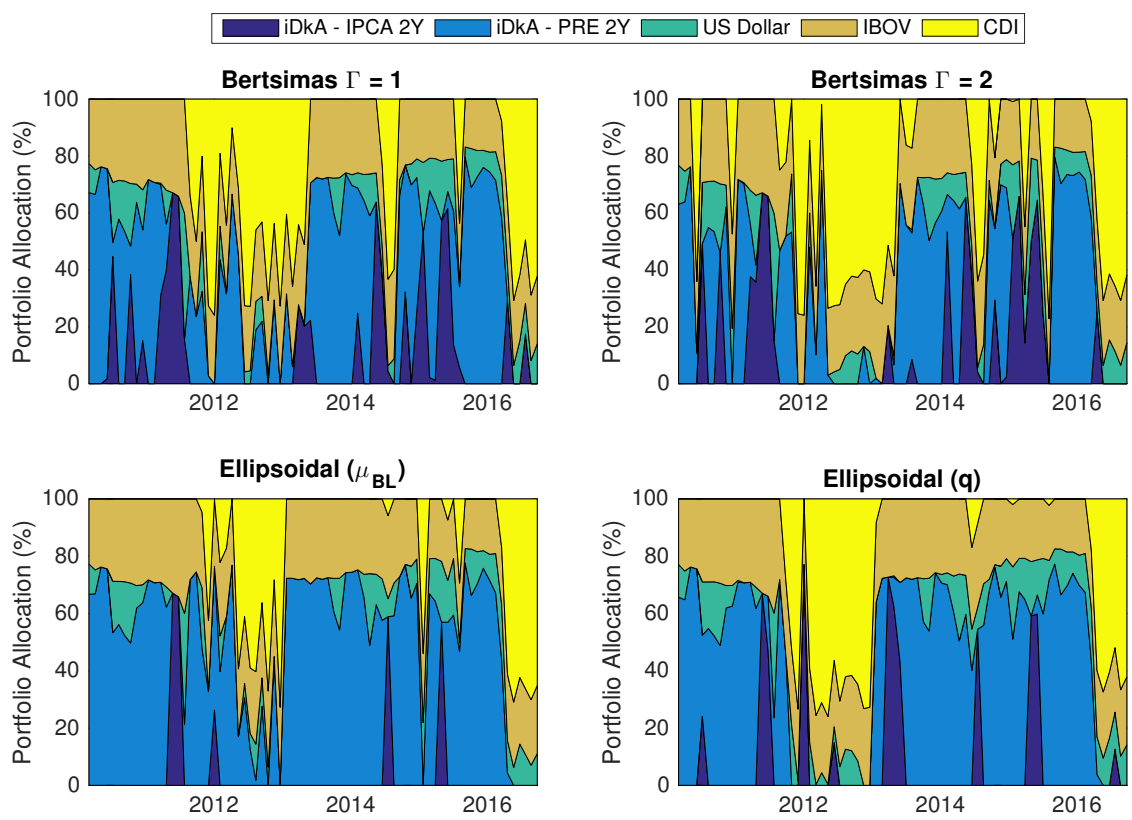

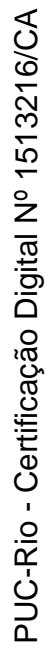
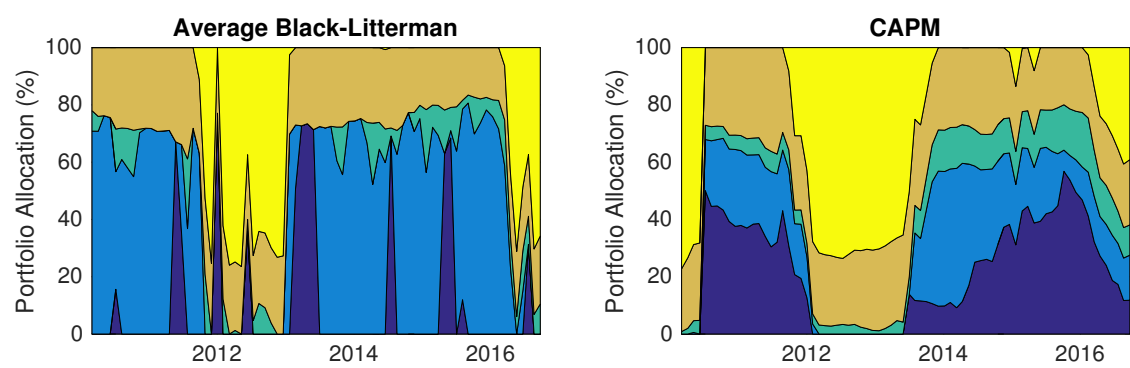

Figure 5.7: Portfolio weights in the period of analysis for $\tau_{c}=1$. 

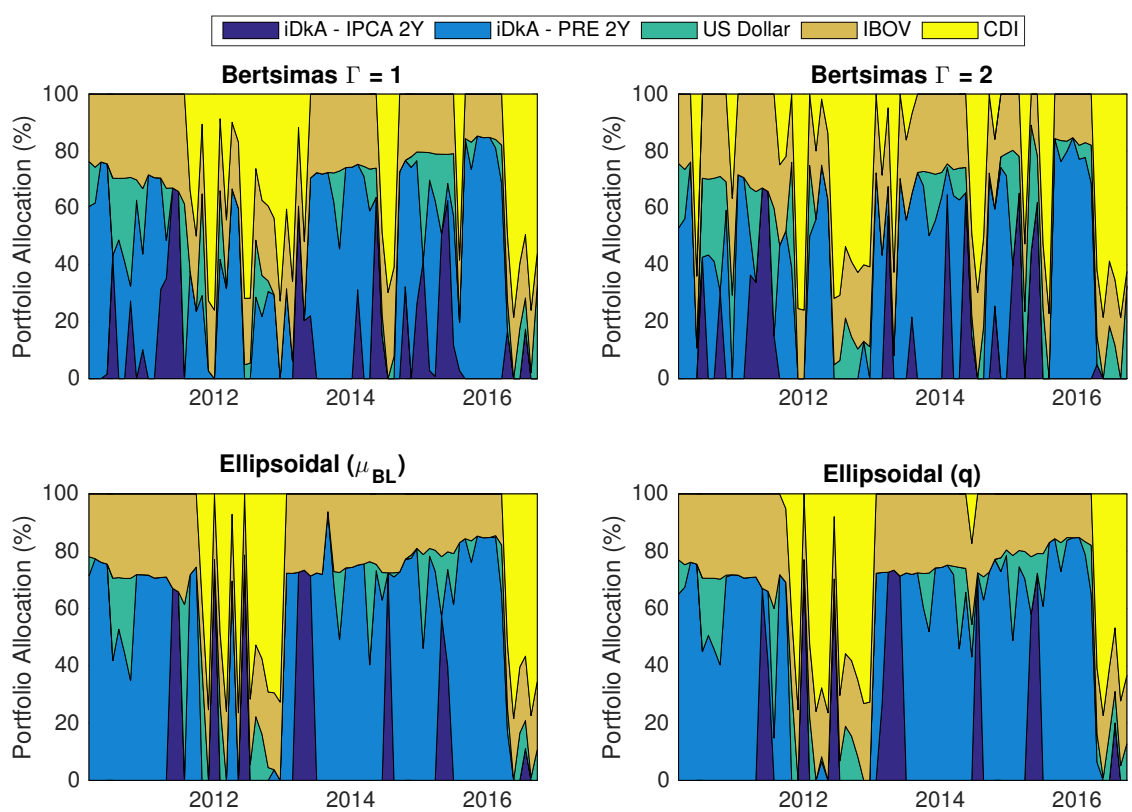

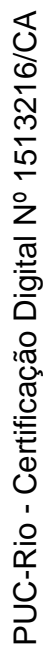
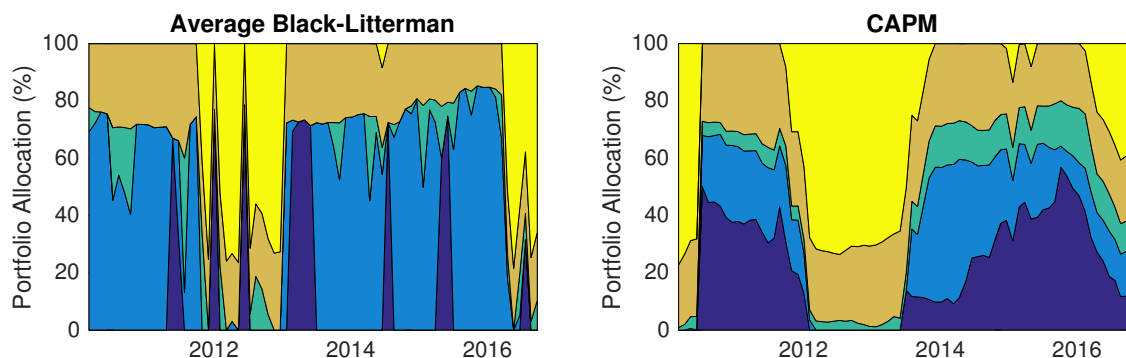

Figure 5.8: Portfolio weights in the period of analysis for $\tau_{c}=10$. 

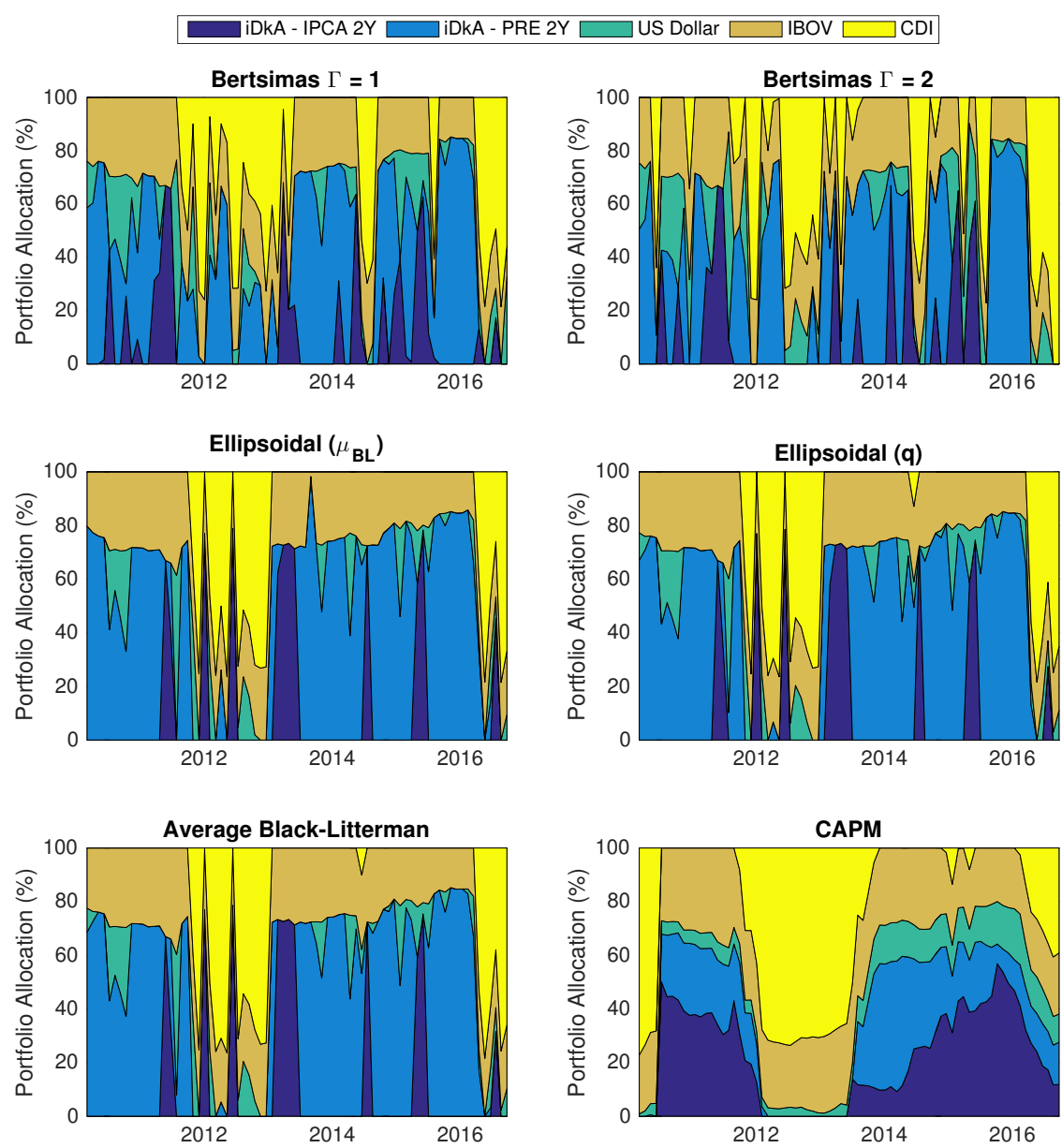

Figure 5.9: Portfolio weights in the period of analysis for $\tau_{c}=100$.

Two interesting observations can be made from these plots. The first is that the views reduced the exposure on US Dollar when the currency started to devalue in the beginning of 2016. In this same period, the reduction on the US Dollar weights are progressively increasing with the paramter $\tau_{c}$ for all models. However, the CAPM equilibrium US Dollar allocation relative to other asset classes remained practically unchanged during 2016. This behavior is expected from a passive strategy. Second, the views tilted the government bonds portfolio to fixed-income government bonds and reduced the allocation on inflation-indexed bonds, which is due to the greater uncertainty on inflation during this period. Nevertheless, during the beginning of 2015 when real yield significantly to outperformed nominal yields all models had most of its government bonds weighs in inflation-indexed bonds. The exceptions here are the robust Bertsimas models which started to allocate in inflation indexed 
bonds in the beginning of 2014 .

The average portfolio weights on our data set are presented in table 5.4. Note that the average allocation on other asset classes changes significantly with both the confidence parameter and the allocation model. We also observe that the average allocation in the risk-free asset is higher on the Bertsimas, which indicates that these models are taking less unnecessary risk due to the conflicting information on the absolute views.

\begin{tabular}{cccccccc}
\hline \hline$\tau_{c}$ & & $\mathrm{~B}(\Gamma=2)$ & $\mathrm{B}(\Gamma=1)$ & EL $\left(\mu_{B L}\right)$ & EL $(q)$ & Av. BL & CAPM \\
\hline \hline \multirow{4}{*}{0.1} & CDI & 0.25 & 0.24 & 0.19 & 0.23 & 0.22 & 0.25 \\
& iDkA - IPCA 2Y & 0.17 & 0.13 & 0.04 & 0.07 & 0.09 & 0.22 \\
& US Dollar & 0.09 & 0.08 & 0.10 & 0.10 & 0.08 & 0.09 \\
& IBOV & 0.26 & 0.26 & 0.26 & 0.26 & 0.25 & 0.26 \\
\hline \multirow{4}{*}{1} & CDI & 0.25 & 0.20 & 0.13 & 0.17 & 0.16 & 0.25 \\
& iDkA - IPCA 2Y & 0.11 & 0.11 & 0.04 & 0.08 & 0.10 & 0.22 \\
& iDkA - PRE 2Y & 0.29 & 0.36 & 0.50 & 0.41 & 0.43 & 0.18 \\
& US Dollar & 0.09 & 0.08 & 0.08 & 0.09 & 0.06 & 0.09 \\
& IBOV & 0.26 & 0.26 & 0.25 & 0.25 & 0.25 & 0.26 \\
\hline \multirow{4}{*}{10} & CDI & 0.22 & 0.19 & 0.15 & 0.16 & 0.15 & 0.25 \\
& iDkA - IPCA 2Y & 0.10 & 0.10 & 0.09 & 0.09 & 0.10 & 0.22 \\
& iDkA - PRE 2Y & 0.32 & 0.37 & 0.45 & 0.43 & 0.43 & 0.18 \\
& US Dollar & 0.11 & 0.09 & 0.07 & 0.07 & 0.06 & 0.09 \\
& IBOV & 0.25 & 0.25 & 0.24 & 0.25 & 0.25 & 0.26 \\
\hline \multirow{4}{*}{100} & CDI & 0.22 & 0.19 & 0.14 & 0.15 & 0.15 & 0.25 \\
& iDkA - IPCA 2Y & 0.10 & 0.10 & 0.10 & 0.10 & 0.10 & 0.22 \\
& iDkA - PRE 2Y & 0.32 & 0.37 & 0.45 & 0.43 & 0.43 & 0.18 \\
& US Dollar & 0.11 & 0.09 & 0.07 & 0.07 & 0.07 & 0.09 \\
& IBOV & 0.24 & 0.25 & 0.24 & 0.25 & 0.25 & 0.26 \\
\hline
\end{tabular}

Table 5.4: Average portfolio weights on the data set for $\tau_{c}=(0.1,1,10,100)$. 

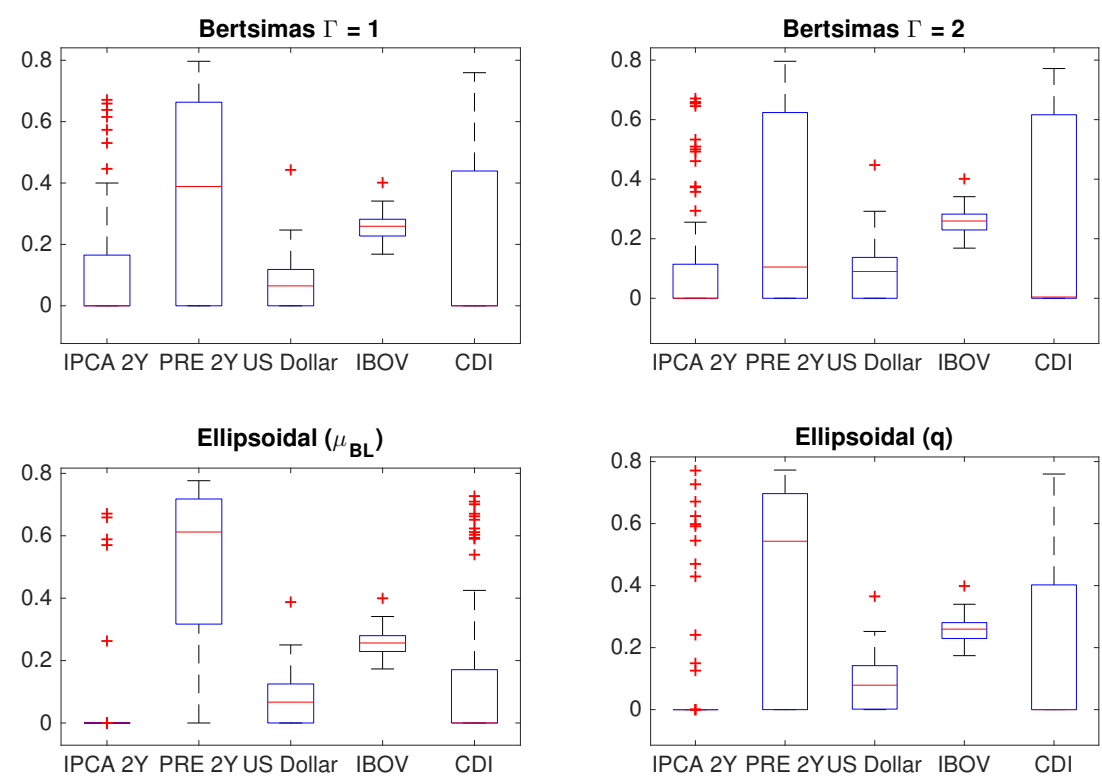

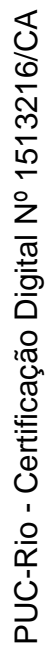
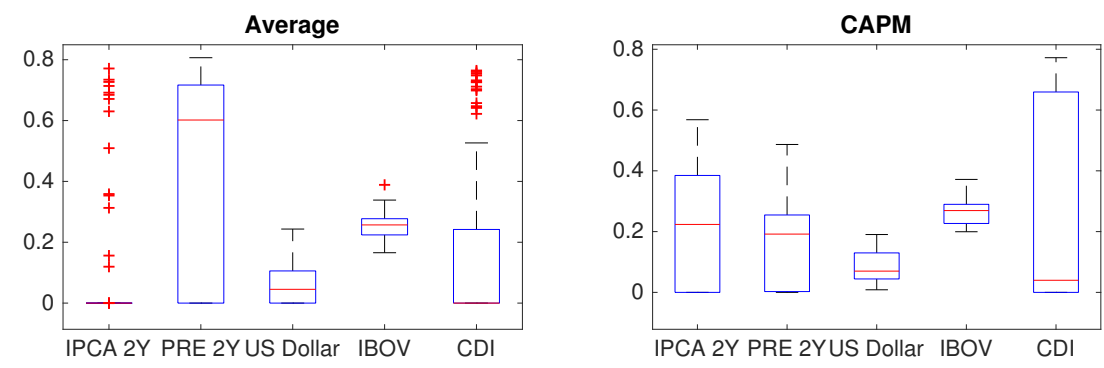

Figure 5.10: Boxplot of the monthly portfolio weights for $\tau_{c}=1$. 

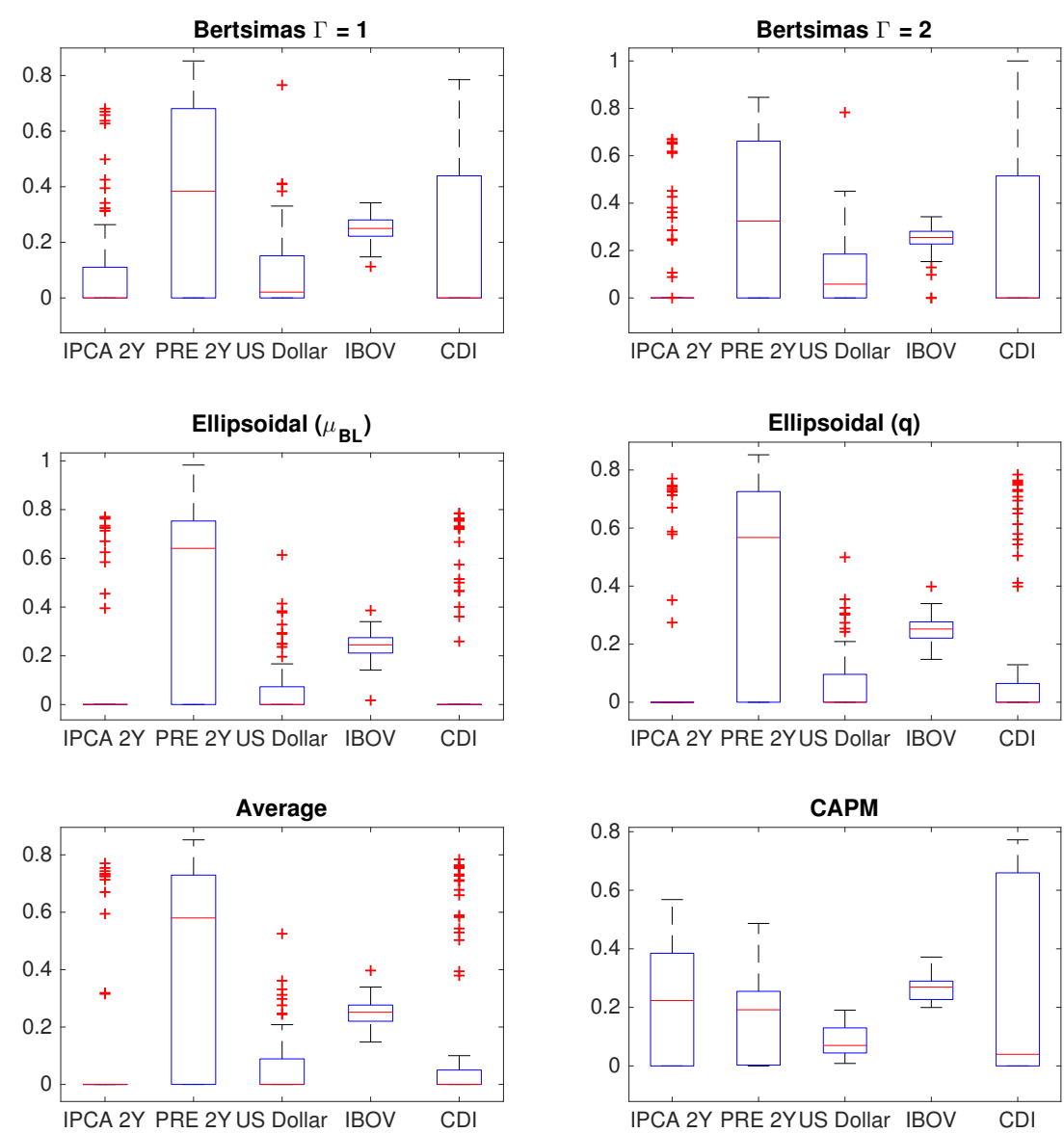

Figure 5.11: Boxplot of the monthly portfolio weights for $\tau_{c}=100$.

Analogous to the study on the out of sample daily returns, in figures 5.10 and 5.11 we present the boxplot of the monthly allocations for all strategies and $\tau_{c}=(1,100)$, in a total of 76 observations. These plots support the following remarks:

(a) The robust strategies using Bertsimas and Sim's uncertainty set remains conservative on the risk-free allocation when $\tau_{c}$ increases. In the case of $\Gamma=2$ there are periods where the portfolio is only allocated in risk-free, which is caused by worst-case pessimistic views on all asset classes.

(b) The statistics of the monthly weights on Ibovespa index is approximate the same for all models and both levels of confidence. The average allocation is also similiar as is shown is table 5.4. This is expected since we are not assuming views on the stock market. However, this shows the ability of 
the Black-Litterman model to impose active views on portfolios and asset classes.

(c) In the average Black-Litterman and ellipsoidal uncertainty sets, the median(red line) on the US Dollar allocation reduces to zero when $\tau_{c}=100$. However, the number of obsevations of weights above $20 \%$ increases for the same asset class. This simple example shows how the portfolio weights of original Black-Litterman is sensitive to the confidence on the views.

Summarizing the numerical results based on our historical data sample, it can be said that robust version of the Black-Litterman model can lead to an added value portfolios compared to the original model. Our out-ofsample evaluation results presented in this section show that the robust BlackLitterman portfolios can substantially outperformed the original model. As we argued in the previous chapter, the reason for this is that estimates of views can be improved when we consider an uncertainty structure instead of a pointwise estimate. In our tests we have seen that the robust models that considered the maximum, minimum and average values of the views have outperformed the ones that only assumed information on the average. Moreover, as we increased the confidence $\left(\tau_{c}\right)$ these models had even better relative results. 


\section{Conclusions}

The aim of the work was to further the understanding of robust asset allocation, in particular, we propose a robust approach to the Black-Litterman to asset allocation model. We have also extended the Black-Litterman methodology using recent developments of robust optimization techniques to introduce conflicting source of input views. The major distinction between the approaches is that the first allows investor to input a single point estimation for the views, whereas the second allows to create a uncertainty sets on these inputs.

Further we studied properties of the original and robust Black-Litterman models for various degrees of accuracy and dispersion of the input views. Through empirical results on synthetic data we have showed situation which the robust model can benefit from this new setting. Computational evidence suggests that the robust approaches provide certain benefits on the performance over the traditional model, especially in scenarios where views are not known with accuracy. We also observed that the robust models are less volatile in two situations, when the forecasters are uncertain about the direction of the market and when the uncertainty sets of the views are large.

We have also tested our models on real market data from the Brazilian financial market. For the views, we used a public data provided by the Central Bank of Brazil. The numerical results suggest that robust version can outperform the original Black-Litterman model when there are conflicting and different sources of information about the views. We have also shown in our out of sample exercise that the portfolio weights of the robust model is less sensitive to the accuracy of the forecasters when we consider the total uncertainty structure of the views.

We believe that these robust formulations, given its simplicity provide a feasible strategy to practitioners incorporate on their Black-Litterman allocations. In addition, for future work one might consider modeling the uncertainty sets in a purely data-drive methodology and test the properties of each model under these circumstances. We also encourage to use other sources of data sets from different countries to further compare the models. There are also several interesting extensions that are worth exploring. The models in this thesis are all related to uncertainty on the views for multiple forecasters, one can also study the impact of the CAPM on the Black-Litterman model and how a robust formulation can help mitigate estimation error and improve performance. 


\section{Bibliography}

ALLAJ, E. The black-litterman model: a consistent estimation of the parameter tau. Financial Markets and Portfolio Management, vol. 27, no. 2, p. 217251, 2013. 2.3.1, 5.4

BARBOSA, A. Central Bank of Brazil's market expectations system: a tool for monetary policy. 2012. Available from Internet: $<$ http://www.bis.org/ifc/publ/ifcb36t.pdf $>$. 5.1

BASAK, G. K.; JAGANNATHAN, R.; MA, T. Jackknife estimator for tracking error variance of optimal portfolios. Management Science, vol. 55, no. 6, p. 990-1002, 2009. 1

BEN-TAL, A.; NEMIROVSKI, A. Robust convex optimization. Mathematics of Operations Research, vol. 23, no. 4, p. 769-805, 1998. 2.1.2, 2.2

BEN-TAL, A.; NEMIROVSKI, A. Robust solutions of uncertain linear programs. Operations Research Letters, vol. 5, no. 1, p. 1 - 13, 1999. ISSN 0167-6377. $2.2,2.2 .1$

BEN-TAL, A.; NEMIROVSKI, A. Robust solutions of linear programming problems contaminated with uncertain data. Mathematical Programming, vol. 88, no. 3 , p. $411-424,2000.2 .2 .3$

BEN-TAL, A.; NEMIROVSKI, A.; ROOS, C. Robust solutions of uncertain quadratic and conic-quadratic problems. SIAM J. on Optimization, Society for Industrial and Applied Mathematics, Philadelphia, PA, USA, vol. 13, no. 2, p. 535-560, 2002. ISSN 1052-6234. 2.1.2

BERTSIMAS, D.; BROWN, D. B. Constructing uncertainty sets for robust linear optimization. Operations Research, vol. 57, no. 6, p. 1483-1495, 2009. Available from Internet: <http://dx.doi.org/10.1287/opre.1080.0646>. 2.2.3

Bertsimas, D.; Gupta, V.; Kallus, N. Data-Driven Robust Optimization. ArXiv e-prints, dec 2014. 2.2 .3

BERTSIMAS, D.; SIM, M. The price of robustness. Operations Research, vol. 52, no. 1 , p. 35-53, 2004. 2.2, 2.2.3, 3.3.1, 3.3.1 
BERTSIMAS, D.; TAKEDA, A. Optimizing over coherent risk measures and nonconvexities: a robust mixed integer optimization approach. Comp. Opt. and Appl., vol. 62, no. 3, p. 613-639, 2015. 2.2 .3

BEST, M. J.; GRAUER, R. R. The analytics of sensitivity analysis for mean-variance portfolio problems. International Review of Financial Analysis, vol. 1, no. 1, p. $17-37,1992.1$

BEVAN, A.; WINKELMANN, K. Using the black-litterman global asset allocation model: Three years of practical experience. 1998. 2.3.1

BIRGE, J. R.; LOUVEAUX, F. Introduction to Stochastic Programming. [S.I.]: Springer, 1997. (Springer Series in Operations Research and Financial Engineering). 2.2

BLACK, F.; LITTERMAN, R. Asset Allocation: Combining Investor Views with Market Equilibrium. The Journal of Fixed Income, 1990. 2.3, 2.3.1

BLACK, F.; LITTERMAN, R. Global Portfolio Optimization. Financial Analysts Journal, CFA Institute, vol. 48, no. 5, 1992. ISSN 0015198X. 1, 2.3, 2.3.1, 2.3.1

CAMPBELL, J. Y. et al. The Econometrics of Financial Markets. Princeton, NJ: Princeton University Press, 1997. 5.2.1

CERIA, S.; STUBBS, R. A. Incorporating estimation errors into portfolio selection: Robust portfolio construction. Journal of Asset Management, Palgrave Macmillan UK, vol. 7, no. 2, p. 109-127, jul. 2006. 2.2.4

CHOPRA, V. K.; ZIEMBA, W. T. The Effect of Errors in Means, Variances, and Covariances on Optimal Portfolio Choice. Journal of Portfolio Management, vol. 19 , no. 2 , p. $6-11,1993.1$

DEMIGUEL, V. et al. A generalized approach to portfolio optimization: Improving performance by constraining portfolio norms. Management Science, vol. 55, no. 5 , p. $798-812,2009.1$

DUNNING, I.; HUCHETTE, J.; LUBIN, M. JuMP: A modeling language for mathematical optimization. arXiv:1508.01982 [math.OC], 2015. Available from Internet: <http://arxiv.org/abs/1508.01982>. 5

ELTON, E. J. et al. Modern portfolio theory and investment analysis. [S.I.]: John Wiley \& Sons, 2009. 2.3.1 
FABOZZI, F.; MANN, S. The Handbook of Fixed Income Securities, Eighth Edition. [S.I.]: McGraw-Hill Education, 2011. ISBN 9780071768474. 5.2

FABOZZI, F. J. Bond Markets, Analysis and Strategies. 8. ed. [S.I.]: Prentice Hall, 2012. 5.2

FABOZZI, F. J.; HUANG, D.; ZHOU, G. Robust portfolios: contributions from operations research and finance. Annals of Operations Research, vol. 176, no. 1 , p. 191-220, 2009. 1, 2.2, 2.2.4

FABOZZI, F. J.; KOLM, D. P. P. N.; FOCARDI, S. M. Robust Portfolio Optimization and Management. 1. ed. [S.I.]: John Wiley, 2007. 1, 2.1, 2.3.3

FERNANDES, B.; FERNANDES, C.; STREET, A. An asset allocation model with inequalities constraints and coherent risk measure: an application to Brazilian equities. Revista de Finanças Aplicadas, p. 1 - 27, 2013. 1

FERNANDES, B. et al. An adaptive robust portfolio optimization model with loss constraints based on data-driven polyhedral uncertainty sets. European Journal of Operational Research, vol. 255, no. 3, p. $961-970,2016$. ISSN 0377-2217. 1

FERNANDES, B. et al. An adaptive robust portfolio optimization model with loss constraints based on data-driven polyhedral uncertainty sets. European Journal of Operational Research, vol. 255, no. 3, p. 961-970, 2016. 2.2.4

GHAOUI, L. E.; LEBRET, H. Robust solutions to least-squares problems with uncertain data. SIAM Journal on Matrix Analysis and Applications, vol. 18, no. 4 , p. $1035-1064,1997.2 .2$

GHAOUI, L. E.; OUSTRY, F.; LEBRET, H. Robust solutions to uncertain semidefinite programs. SIAM Journal on Optimization, vol. 9, no. 1, p. 33-52, 1998. 2.2

HALLDÓRSSON, B.; TÜTÜNCÜ, R. An interior-point method for a class of saddle-point problems. Journal of Optimization Theory and Applications, vol. 116 , no. 3 , p. 559-590, 2003. 1

HE, G.; LITTERMAN, R. The intuition behind black-litterman model portfolios. Goldman Sachs Asset Management Working paper, 1999. 2.3.1, 1, 3, 2

HE, G.; LITTERMAN, R. The intuition behind black-litterman model portfolios. Available at SSRN 334304, 2002. 1 
HEROLD, U. Computing implied returns in a meaningful way. Journal of Asset Management, vol. 6 , no. 1 , p. $53-64$, June 2005. ISSN 1470-8272. 1

IDZOREK, T. A step-by-step guide to the black-litterman model. Forecasting Expected Returns in the Financial Markets, p. 17, 2002. 1

IDZOREK, T. A step-by-step guide to the black-litterman model. In Forecasting Expected Returns in the Financial Markets. [S.I.]: Elsevier, 2007. p. $17-38.2 .3$

JORION, P. Bayes-stein estimation for portfolio analysis. Journal of Financial and Quantitative Analysis, vol. 21, no. 03, p. 279-292, 1986. 1

KIM, J. H.; KIM, W. C.; FABOZZI, F. J. Recent developments in robust portfolios with a worst-case approach. Journal of Optimization Theory and Applications, vol. 161 , no. 1 , p. 103-121, 2013. 1, 2.2 .4

KOIVU, M.; PENNANEN, T. Return dynamics of index-linked bond portfolios. Journal of Portfolio Management, vol. 41, no. 4, p. 78-84, 2014. 5.2

LOBO, M.; BOYD, S. The worst-case risk of a portfolio. Technical Report, Stanford University, 2000. 1

LOBO, M. S. et al. International linear algebra society (ilas) symposium on fast algorithms for control, signals and image processing applications of second-order cone programming. Linear Algebra and its Applications, vol. 284, no. 1, p. 193 - 228, 1998. ISSN 0024-3795. 2.1

LUENBERGER, D. Investment Science. [S.I.]: Oxford University Press, 1998. 5.2 .1

LUTGENS, F. J. W. Hochschulschrift, Dissertation, Thesis, Robust portfolio optimization. 2004. Zsfassung in niederländ. Sprache. 2.1

MANKERT, C. The Black-Litterman Model : Towards its use in practice. 230 p. Tese (Doutorado) - KTH, Management Control, 2010. QC 20101202. 1

MARKOWITZ, H. Portfolio selection. The Journal of Finance, Blackwell Publishing for the American Finance Association, vol. 7, no. 1, p. 77-91, Março 1952. 1

MEUCCI, A. Risk and Asset Allocation. [S.I.]: Springer Finance, 2005. 5.3 
MEUCCI, A. The Black-Litterman Approach: Original Model and Extensions. Social Science Research Network Working Paper Series, SSRN, apr. 2008. $1,2.3,2.3 .1,2.3 .2,2.3 .2,2.3 .4,2.3 .4$

NATARAJAN, K.; PACHAMANOVA, D.; SIM, M. Constructing risk measures from uncertainty sets. Operations Research, vol. 57, no. 5, p. 1129-1141, 2009. Available from Internet: <http://dx.doi.org/10.1287/opre.1080.0683>. 2.2 .3

NEMIROVSKI, A. Lectures on Robust Convex Optimization. [s.n.], 2012. Available from Internet: <http://www2.isye.gatech.edu/ nemirovs/>. 2.2.1

NEMIROVSKI, A. Lectures on Modern Convex Optimization. [s.n.], 2013. Available from Internet: <http://www2.isye.gatech.edu/ nemirovs/>. 2.1

PACHAMANOVA, D. A.; FABOZZI, F. J. Simulation and Optimization in Finance: Modeling with MATLAB, QRISK, or VBA. [S.I.]: John Wiley e Sons, 2011. ISBN 9781118267752. 2.3.1

SATCHELL, S.; SCOWCROFT, A. A demystification of the Black-Litterman model: Managing quantitative and traditional portfolio construction. Journal of Asset Management, vol. 1, no. 2, p. 138-150, sep. 2000. ISSN 1470-8272. $1,2.3 .1$

SHAPIRO, A.; DENTCHEVA, D.; RUSZCZYNSKI, A. Lectures on Stochastic Programming. [S.I.]: Society for Industrial and Applied Mathematics, 2009. 2.2

SHARPE, W. F. Capital asset prices: A theory of market equilibrium under conditions of risk*. The journal of finance, Wiley Online Library, vol. 19, no. 3 , p. $425-442,1964.2 .3 .1$

SILVA, T.; PINHEIRO, P. R.; POGGI, M. A more human-like portfolio optimization approach. European Journal of Operational Research, vol. 256, no. 1, p. $252-260,2017$. ISSN 0377-2217. 1

SOYSTER, A. L. Technical note-convex programming with set-inclusive constraints and applications to inexact linear programming. 1973. 2.2, 2.2.4, 3.3.1

STURM, J. F.; ZHANG, S. On cones of nonnegative quadratic functions. Mathematics of Operations Research, vol. 28, no. 2, p. 246-267, 2003. 2.1 .2

TABAK, B.; ANDRADE, S. Testing the Expectations Hypothesis in the Brazilian Term Structure of Interest Rates. [S.I.], 2001. 5.2.1

THEIL, H. Principles of econometrics. New York, London, Sydney: John Wiley, 1971. 2.3 .3 
WALTERS, J. The Black-Litterman Model in Detail. Social Science Research Network Working Paper Series, SSRN, 2009. 2.3

WALTERS, J. The black-litterman model in detail. Available at SSRN 1314585 , 2011. 1 
A

Tables from Synthetic Data Experiments

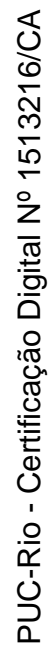




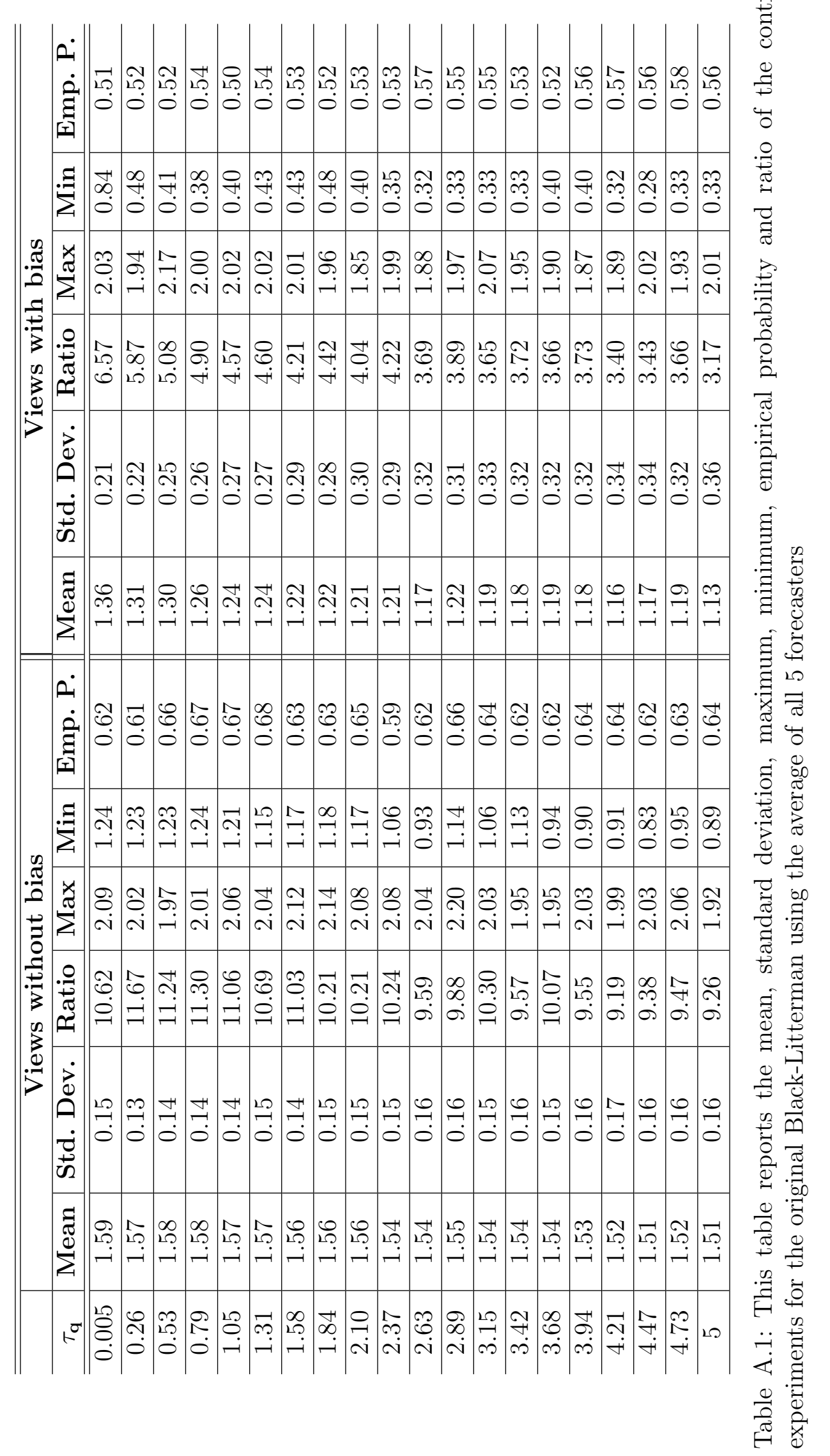




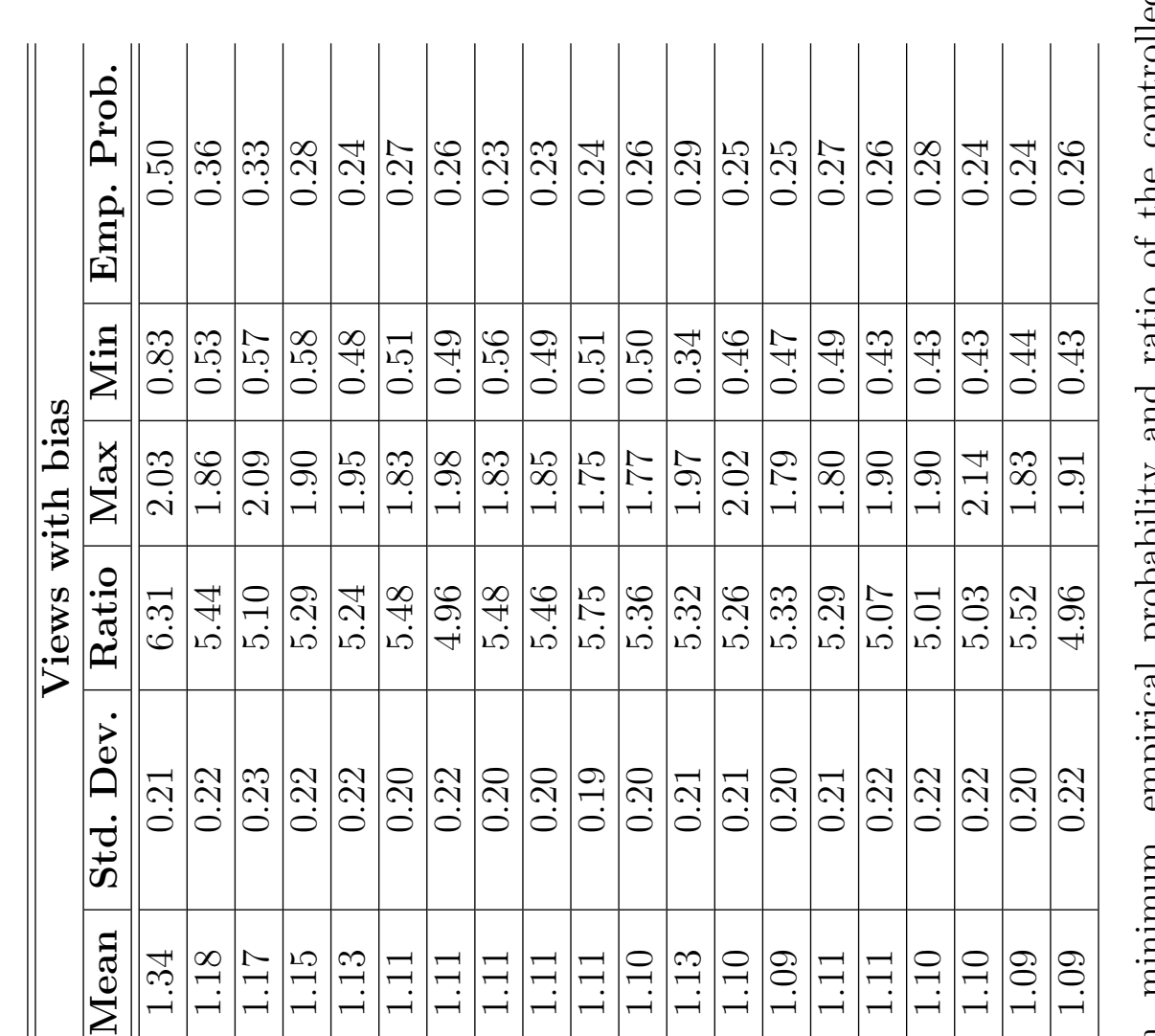

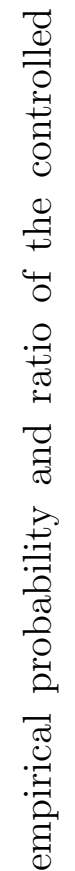

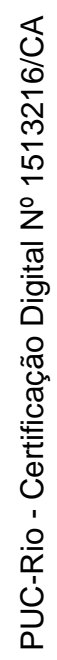

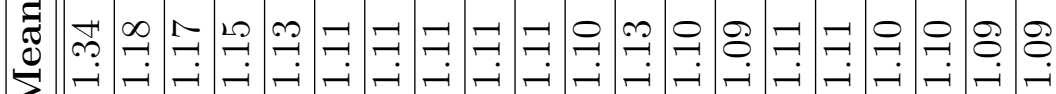

فํำ

는

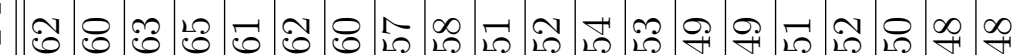

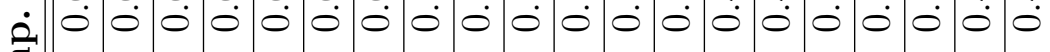

J

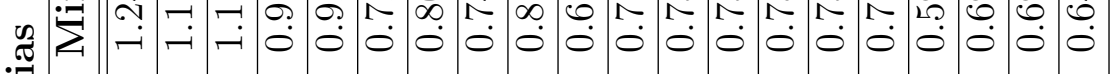

பี

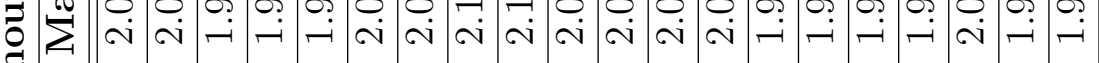

赵

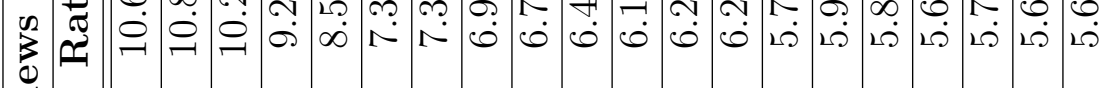

它

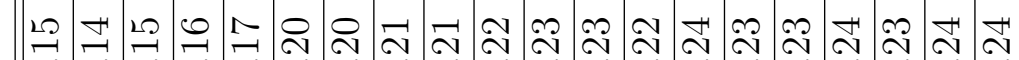

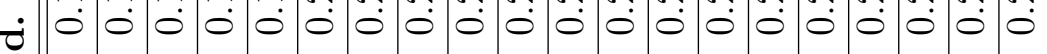
ज

สี

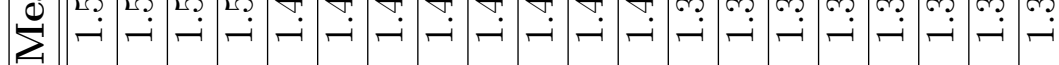

R

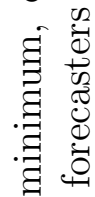




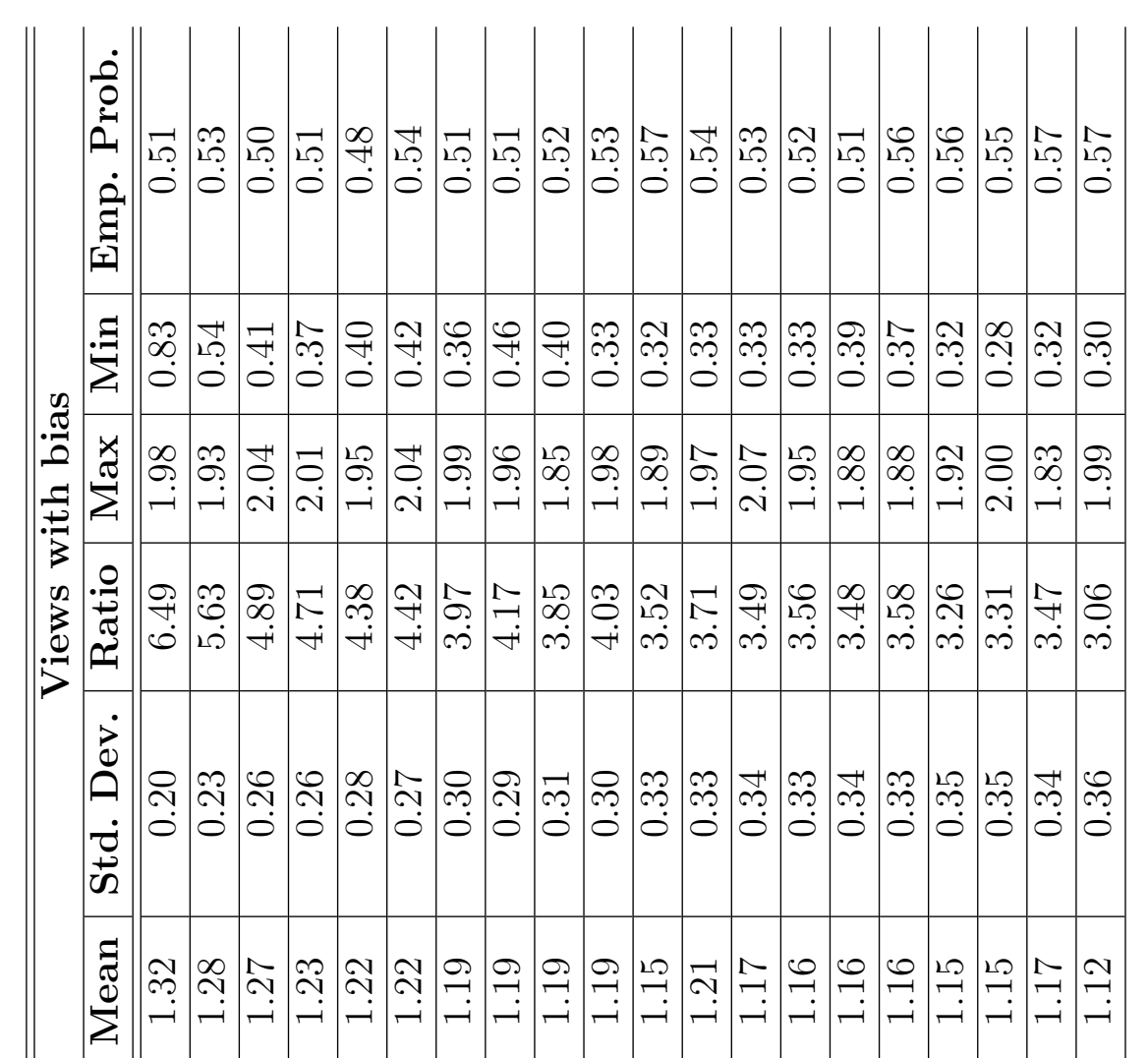

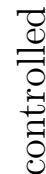

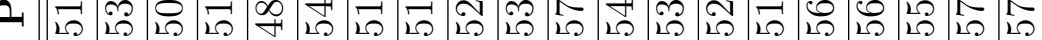

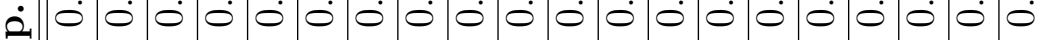

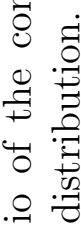
急 赵 풀 :

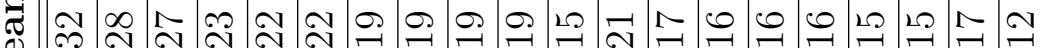
$\dot{0}$

는

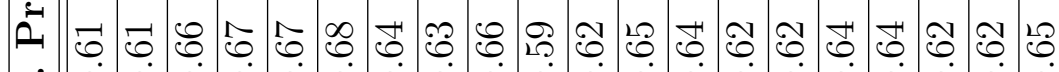

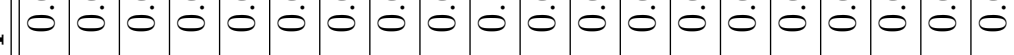

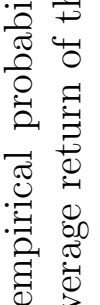

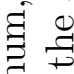

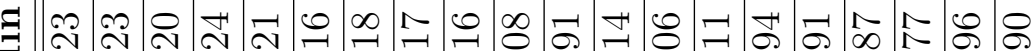

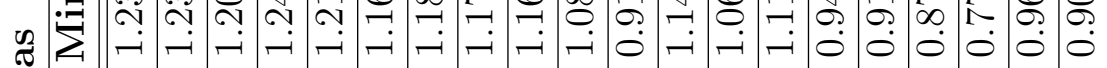

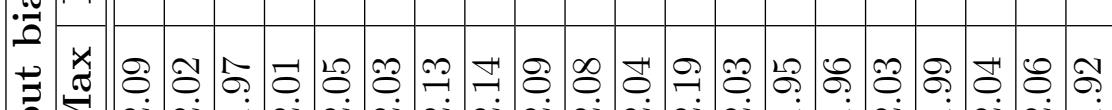

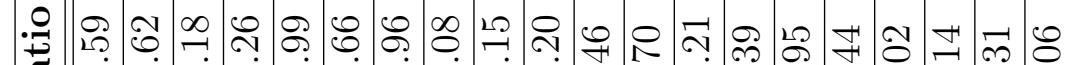
虽

$\stackrel{\oplus}{ }$

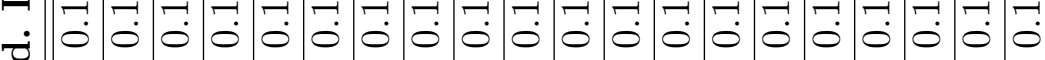
苛

สี

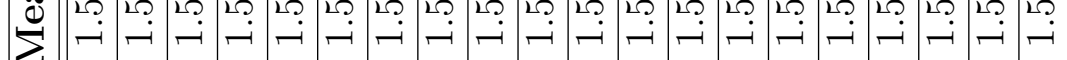
L . 它 륭 สี क. ⿷匚: 巴ँ $\Xi$ 要 똔 की 넨 은 画 . is ४ 远 


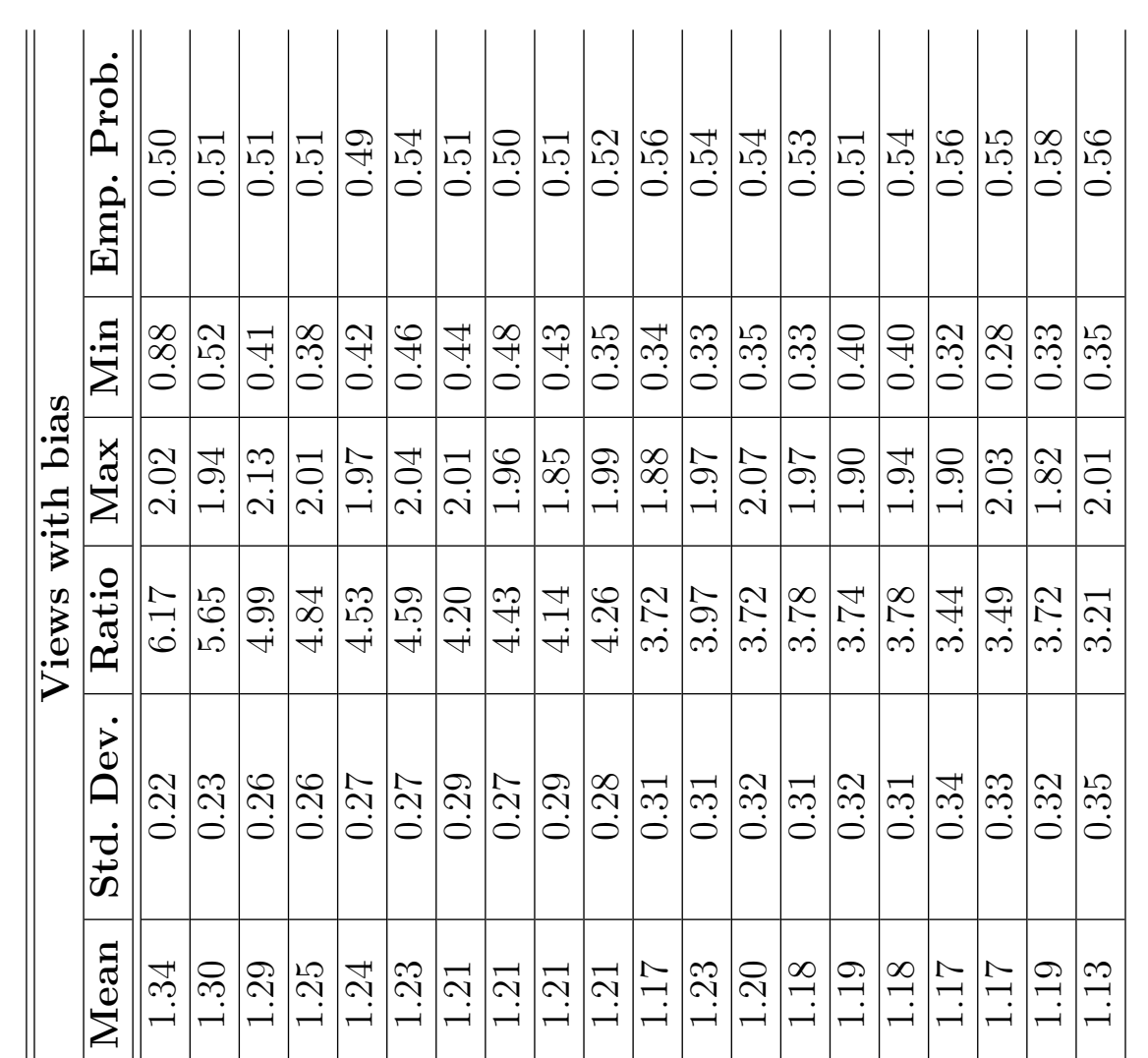

范
0
0
0
0
0 $\stackrel{0}{\leftrightarrows}$ प्र $\stackrel{\circ}{\frac{0}{\pi}}$ $\widetilde{\widetilde{\tau}}$ :

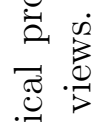
$\infty_{0}^{\infty}$

응

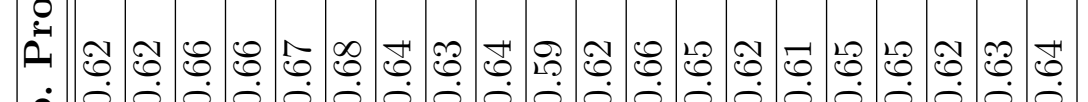

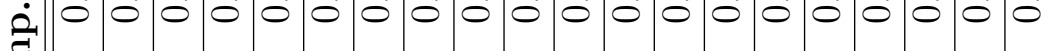

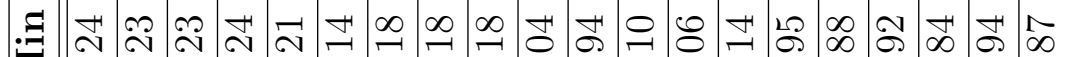

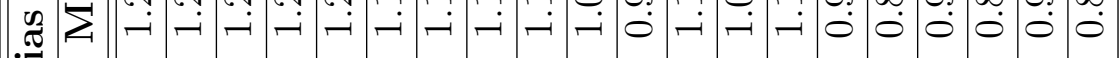

*

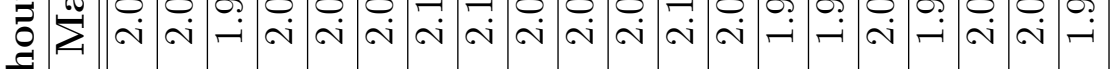

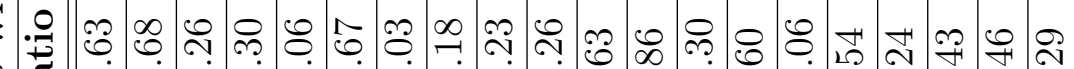

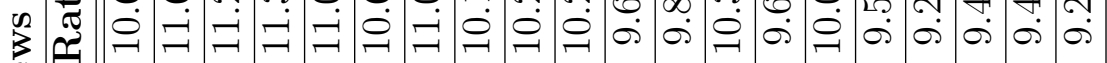

$>$

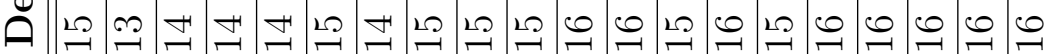

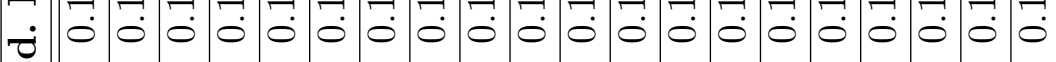
ॠ

สี

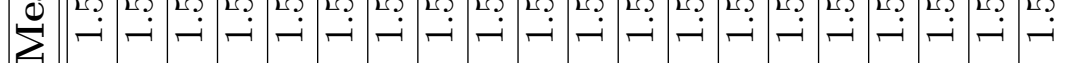

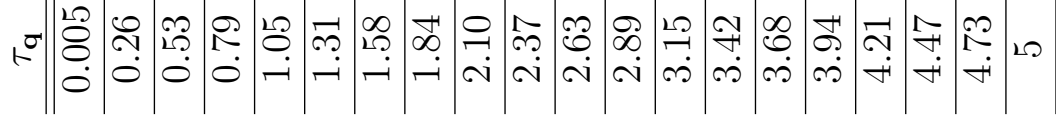




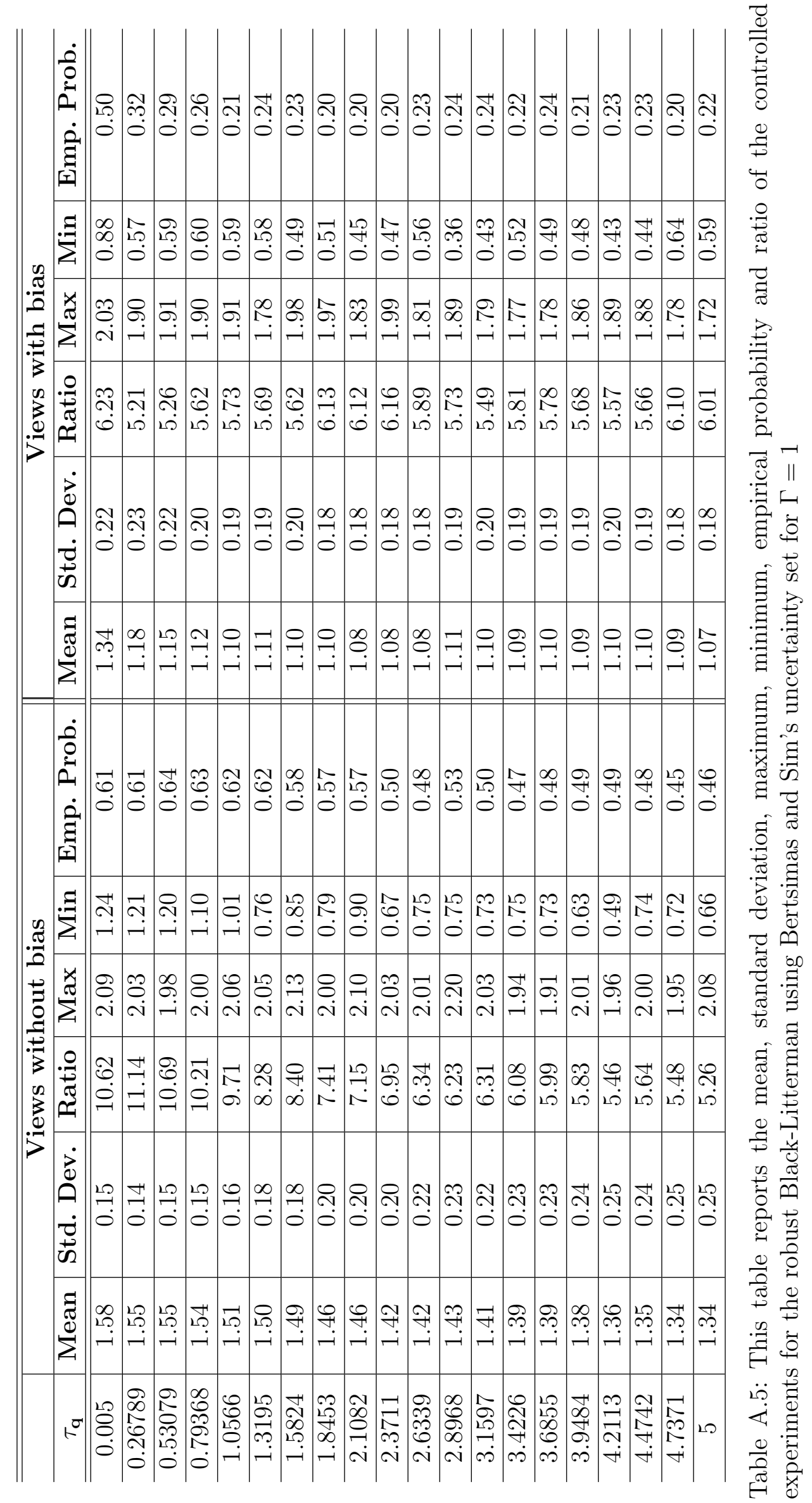




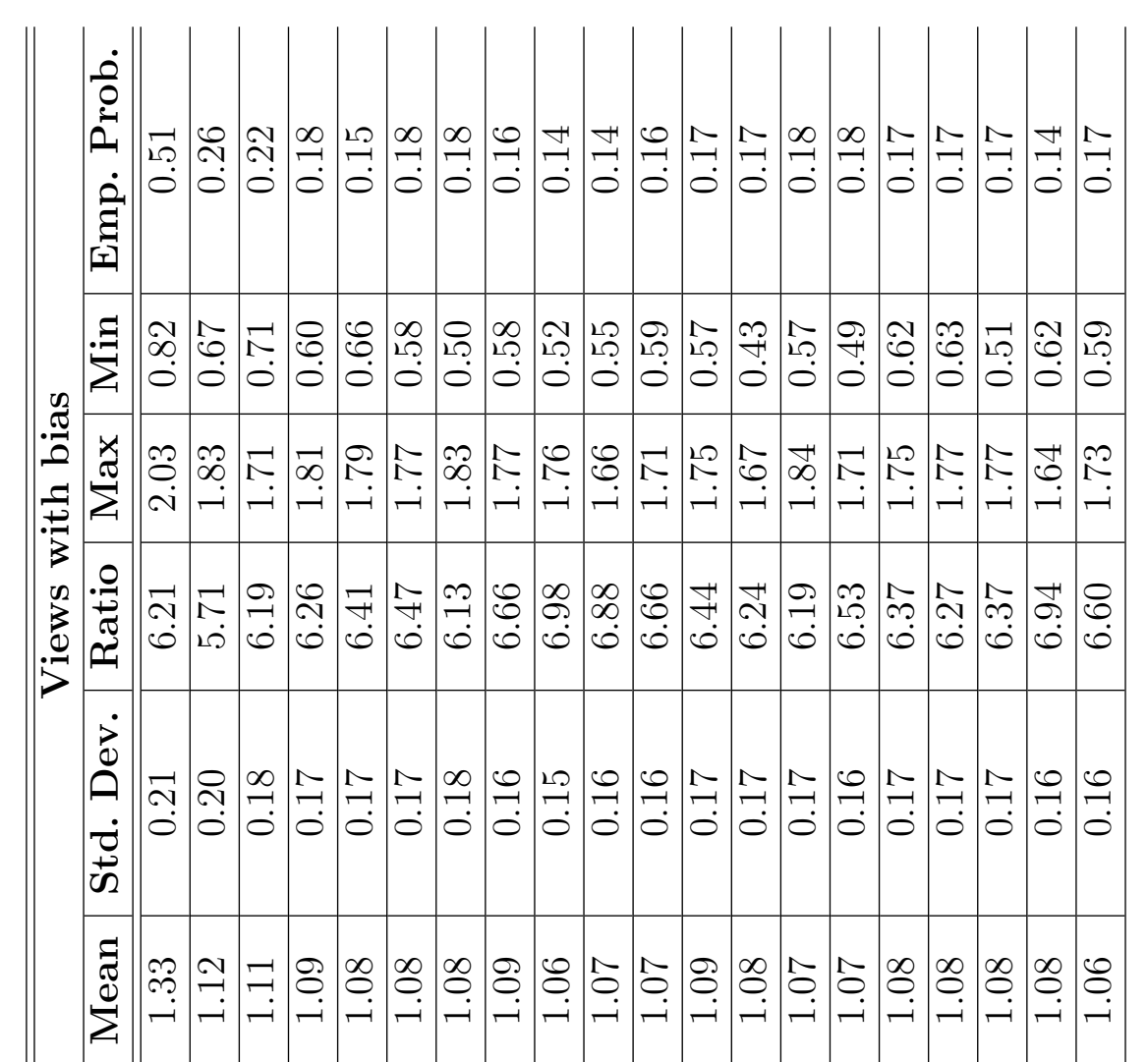

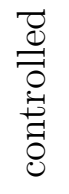

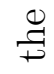

$\breve{0}$

赵

$\widetilde{\widetilde{\tau}}$

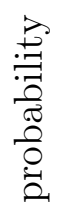

$\stackrel{\mathcal{C}^{*}}{N} \|$

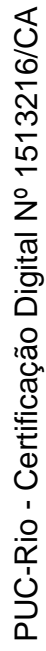

$\therefore$

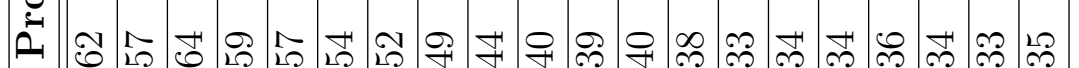

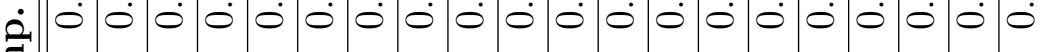

z

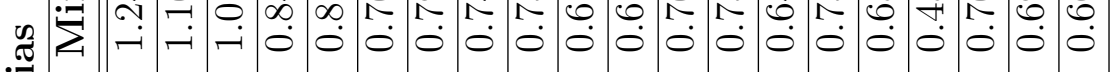

ส

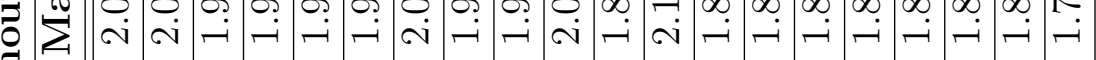

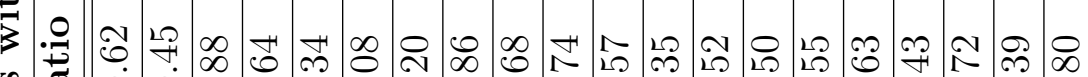

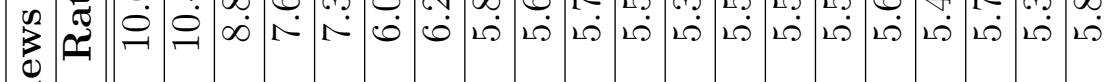

它

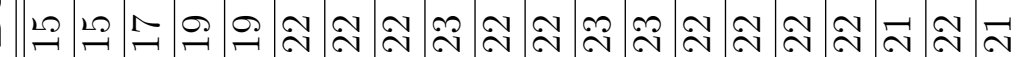

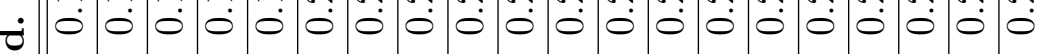
$\ddot{\omega}$

ซี

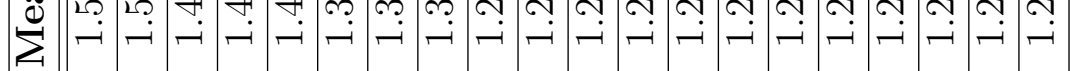

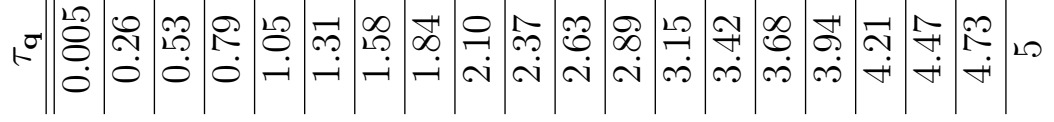

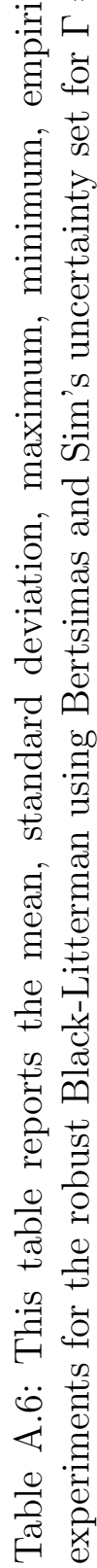




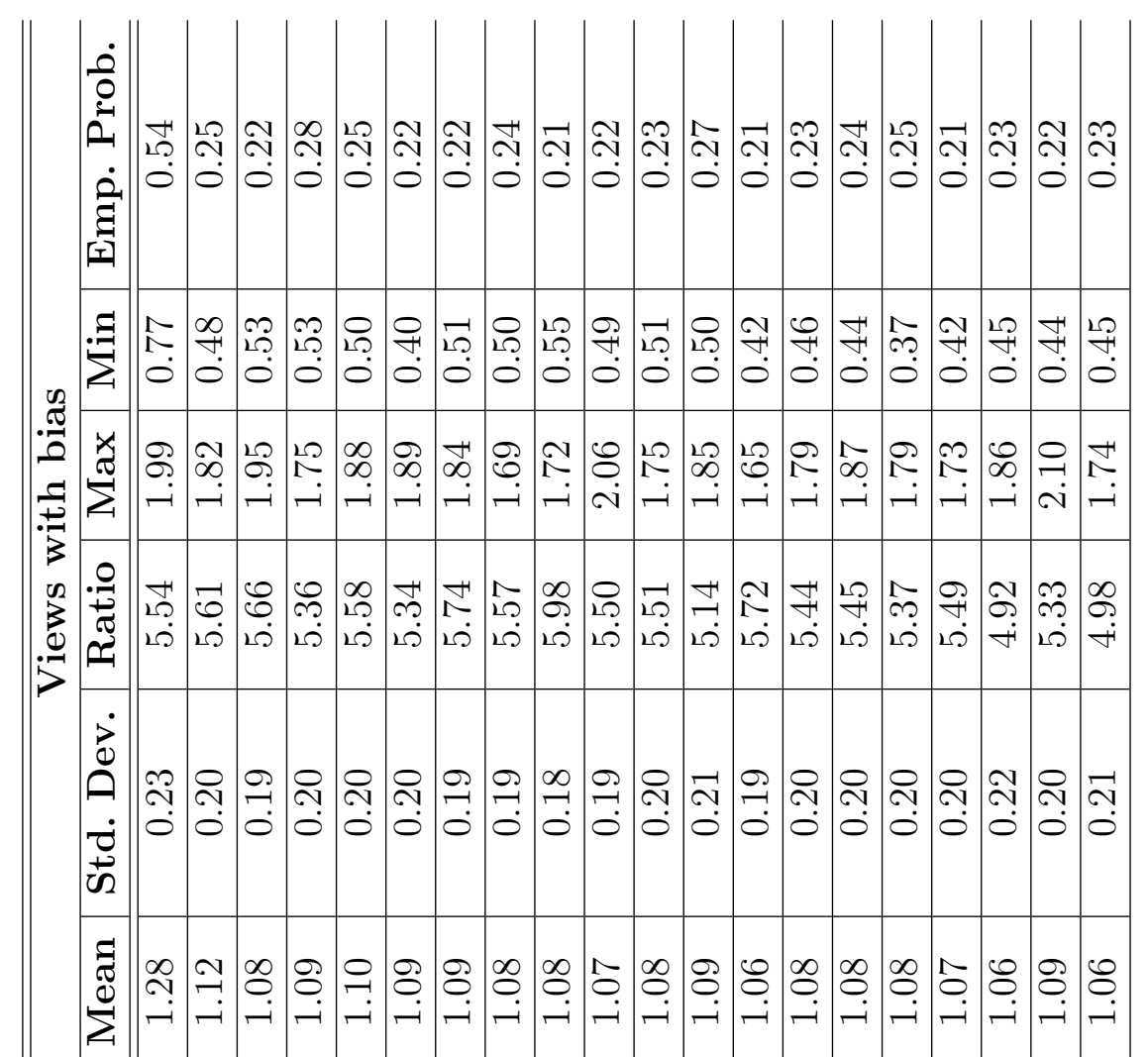

물

تृ.

¿

$B$ 웅

贾

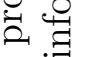

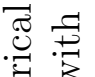

ฮี

응

D

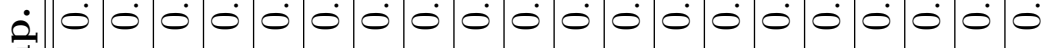

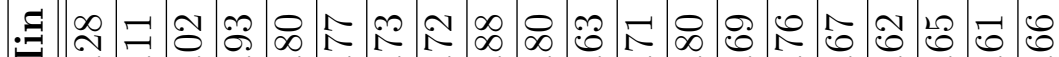

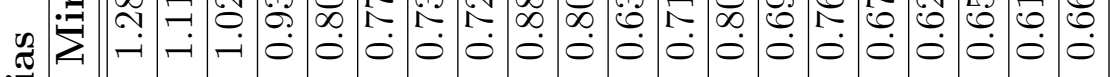
ق

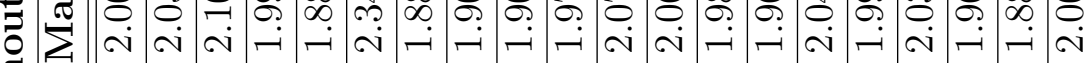

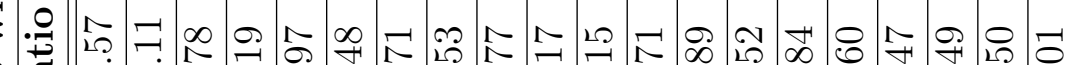
里

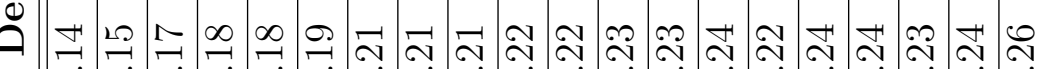

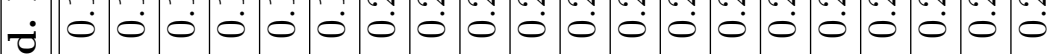
$\ddot{\omega}$

ซี

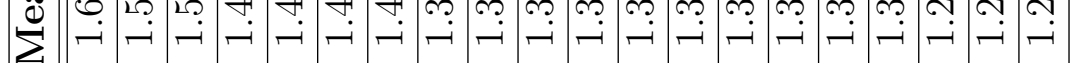
L 